\title{
Performance of the missing transverse momentum triggers for the ATLAS detector during Run-2 data taking
}

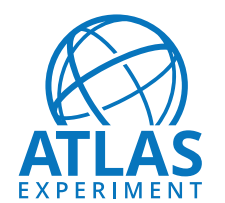

\section{The ATLAS collaboration}

\section{E-mail: atlas.publications@cern.ch}

ABSTRACT: The factor of four increase in the LHC luminosity, from $0.5 \times 10^{34} \mathrm{~cm}^{-2} \mathrm{~s}^{-1}$ to $2.0 \times 10^{34} \mathrm{~cm}^{-2} \mathrm{~s}^{-1}$, and the corresponding increase in pile-up collisions during the $2015-$ 2018 data-taking period, presented a challenge for the ATLAS trigger, particularly for those algorithms that select events with missing transverse momentum. The output data rate at fixed threshold typically increases exponentially with the number of pile-up collisions, so the legacy algorithms from previous LHC data-taking periods had to be tuned and new approaches developed to maintain the high trigger efficiency achieved in earlier operations. A study of the trigger performance and comparisons with simulations show that these changes resulted in event selection efficiencies of $>98 \%$ for this period, meeting and in some cases exceeding the performance of similar triggers in earlier run periods, while at the same time keeping the necessary bandwidth within acceptable limits.

KEYWORDS: Hadron-Hadron scattering (experiments)

ARXIV EPRINT: 2005.09554 


\section{Contents}

1 Introduction 1

2 ATLAS detector 3

3 Description of the $E_{\mathrm{T}}^{\text {miss }}$ trigger algorithms 4

3.1 Level-1 trigger 5

3.2 Trigger using calorimeter cell signals (cell) 6

3.3 Trigger using topological clusters of calorimeter cells (tc_lcw) 6

3.4 Trigger based on jets (mht) 6

3.5 Trigger implementing local pile-up suppression (pufit) 7

4 Offline object and $E_{\mathrm{T}}^{\text {miss }}$ reconstruction $\quad 7$

$5 \quad E_{\mathrm{T}}^{\text {miss }}$ trigger performance $\quad 8$

5.1 Background model based on detector resolution 8

$\begin{array}{ll}5.2 \text { Level-1 trigger performance } & 11\end{array}$

$\begin{array}{lll}5.3 & \text { High-level trigger performance } & 12\end{array}$

$\begin{array}{ll}5.4 \text { Trigger menu evolution and performance } & 16\end{array}$

$\begin{array}{ll}\text { 5.5 Algorithm computation times } & 18\end{array}$

$\begin{array}{ll}\text { 5.6 Dependence on event characteristics } & 19\end{array}$

5.7 Comparison with Monte Carlo simulation 21

6 Conclusion 23

A Full definition of the trigger implementing local pile-up suppression $\quad 24$

$\begin{array}{ll}\text { B Details of the offline reconstruction algorithms } & 27\end{array}$

C The cell $E_{\mathrm{T}}^{\text {miss }}$ background distribution model 28

The ATLAS collaboration $\quad 35$

\section{Introduction}

The trigger system [1] of the ATLAS experiment [2] is responsible for deciding which proton-proton $(p p)$ bunch-crossing events are kept for later analysis. Storage and processing requirements limit the fraction of events that can be retained to the order of $10^{-5}$, with the rest being discarded and hence unavailable for further physics analysis. 
Particles that interact via neither the strong nor the electromagnetic force, and that escape the experiment without decaying, leave no visible signature. Efficient trigger selection of events that contain such invisible particles is nevertheless essential for much of the ATLAS physics programme. Examples include searches for decays of the Higgs boson into invisible final states [3, 4], searches for new charged Higgs bosons decaying into $\tau \nu$ [5], searches for dark matter based on, for example, events in which invisible particles recoil against a single energetic jet [6], supersymmetry searches that involve a stable and invisible neutralino [7, 8], top-quark scalar partner searches [9] and searches for final states with stable long-lived particles [10]. Another recent example is the Standard Model (SM) Higgs boson decay into $b$-quarks [11], a process first observed in events in which the Higgs boson was produced in association with a $Z$ boson which itself decayed into unobserved neutrinos.

Selecting events that contain invisible particles is particularly difficult, precisely because such particles do not register in the detector. The strategy employed is to deduce the presence of these invisible particles from the apparent imbalance of the momentum calculated from the visible particles. In practice the imbalance in the direction parallel to the proton beams is not sensitive since the fraction of each proton's momentum that participates in the collision is unknown, and much of the outgoing momentum in the beam direction is not observed. Instead, the momentum imbalance in the plane perpendicular to the proton beams is the quantity of most interest; it is known as the missing transverse momentum, and its magnitude is conventionally denoted by $E_{\mathrm{T}}^{\text {miss }}$.

The $E_{\mathrm{T}}^{\text {miss }}$ triggers used by ATLAS are based on transverse momentum imbalance within the calorimeter only. Muons are approximately invisible in the calorimeter [12], and so are treated in these calculations much like neutrinos. Neglecting muons results in a negligible cost in terms of additional trigger rate since events containing muons with large transverse momentum are rare. These calorimeter-only algorithms also have the advantage that they can efficiently select events that contain high- $p_{\mathrm{T}}$ muons. For example, the $E_{\mathrm{T}}^{\text {miss }}$ trigger is also used to select events in which the Higgs boson is produced in association with a $Z$ boson decaying into muons, or events containing a $W$ boson decaying into $\mu \nu$ [11].

Given that the selection of events by the $E_{\mathrm{T}}^{\text {miss }}$ trigger is based on energy deposited throughout the calorimeter, there are particular reconstruction challenges. ATLAS employs a trigger system that uses a region-of-interest trigger strategy [1] where the lowest-level trigger identifies potentially interesting objects in each event and, for those events that satisfy the selection criteria, it provides regions of interest to be further analysed by the higher-level trigger. This technique of reconstructing objects only in particular regions of the detector is useful for simplifying the computational task, but generally unsuited to $E_{\mathrm{T}}^{\mathrm{miss}}$ triggers which must sum momenta over the full solid angle that is instrumented.

The most significant challenge to the $E_{\mathrm{T}}^{\text {miss }}$ triggers during the $13 \mathrm{TeV}$ Run-2 datataking period (2015-2018) was the factor of four increase in the number of proton-proton collisions occurring within each bunch crossing. The additional collisions, known as pile-up, were a consequence of the corresponding increase in LHC luminosity from $0.5 \times 10^{34} \mathrm{~cm}^{-2} \mathrm{~s}^{-1}$ in 2015 to $2.0 \times 10^{34} \mathrm{~cm}^{-2} \mathrm{~s}^{-1}$ in 2017 and 2018 . The peak luminosity of $2.0 \times 10^{34} \mathrm{~cm}^{-2} \mathrm{~s}^{-1}$ was achieved with 2544 bunches of circulating protons, a mean number of $p p$ interactions per bunch crossing $\langle\mu\rangle=56$, and a peak pile-up of 70 interactions. The energy from the ad- 
ditional pile-up collisions is deposited throughout the detector. Due to the shaping time of the front-end electronics, the calorimeter response is affected by pile-up from several preceding bunch crossings [13]. The overall effect of both forms of pile-up is to degrade the $E_{\mathrm{T}}^{\mathrm{miss}}$ resolution of the detector. With the existing Run-1 algorithms, this rise in pile-up would have led to an unacceptable order-of-magnitude increase in trigger rate unless thresholds were raised, and that would in turn have significantly diminished the signal efficiencies.

This paper describes algorithms introduced during Run 2 that provide greater pile-up resilience and background rejection while maintaining a signal acceptance similar to that in Run 1. These algorithms were able to keep output rates within a tolerable $100 \mathrm{~Hz}$ even at $\langle\mu\rangle=56$. The design of these algorithms is described in detail, and comparative studies of their performance using data and simulation are provided.

The paper is organized as follows. Section 2 describes the ATLAS detector. The $E_{\mathrm{T}}^{\text {miss }}$ trigger algorithms are introduced in section 3 . The offline $E_{\mathrm{T}}^{\text {miss }}$ algorithm against which the trigger is compared is defined in section 4 . The trigger performance studies and their results are described in section 5 . The conclusions are presented in section 6 .

\section{ATLAS detector}

The ATLAS detector [2] at the LHC covers nearly the entire solid angle around the collision point. It consists of an inner tracking detector surrounded by a thin superconducting solenoid, electromagnetic and hadronic calorimeters, and a muon spectrometer incorporating three large superconducting toroidal magnet systems.

The inner-detector system is immersed in a $2 \mathrm{~T}$ axial magnetic field and provides charged-particle tracking in the range $|\eta|<2.5 .^{1}$ The high-granularity silicon pixel detector covers the collision vertex region [14]. It is followed by the silicon microstrip tracker. These silicon detectors are complemented by the transition radiation tracker.

The calorimeter system has approximately 188,000 cells and covers the pseudorapidity range $|\eta|<4.9$. Within the region $|\eta|<3.2$, electromagnetic calorimetry is provided by barrel and endcap high-granularity lead/liquid-argon (LAr) sampling calorimeters (ECAL), with an additional thin LAr presampler covering $|\eta|<1.8$ to correct for energy loss in material upstream of the calorimeters. The ECAL is between 24 and 27 radiation lengths $\left(X_{0}\right)$ deep, and its granularity in the barrel in terms of $\Delta \eta \times \Delta \phi$ is typically $0.025 \times \pi / 128$, with variations in segmentation with layer and $|\eta|$ as described in ref. [13].

Hadronic calorimetry is provided by the steel/scintillator-tile calorimeter (HCAL), segmented into three barrel structures within $|\eta|<1.7$, and two copper/LAr hadronic endcap calorimeters. The solid angle coverage is completed with forward copper/LAr and tungsten/LAr calorimeter modules (FCAL) optimized for electromagnetic (FCAL1) and hadronic (FCAL2 and FCAL3) measurements respectively. The combined depth of the

\footnotetext{
${ }^{1}$ ATLAS uses a right-handed coordinate system with its origin at the nominal interaction point (IP) in the centre of the detector and the $z$-axis along the beam pipe. The $x$-axis points from the IP to the centre of the LHC ring, and the $y$-axis points upwards. Cylindrical coordinates $(r, \phi)$ are used in the transverse plane, $\phi$ being the azimuthal angle around the $z$-axis. The pseudorapidity is defined in terms of the polar angle $\theta$ as $\eta=-\ln \tan (\theta / 2)$. Angular separation is measured in units of $\Delta R \equiv \sqrt{(\Delta \eta)^{2}+(\Delta \phi)^{2}}$.
} 
calorimeters for hadronic energy measurements is more than 10 nuclear interaction lengths nearly everywhere across the full detector acceptance $(|\eta|<4.9)$. The granularity is as fine as $0.1 \times \pi / 32$, again with variations in segmentation with layer and $|\eta|$ as described in ref. [13].

The muon spectrometer comprises separate trigger and high-precision tracking chambers measuring the deflection of muons in a magnetic field generated by the superconducting air-core toroids. The field integral of the toroids ranges between 2.0 and $6.0 \mathrm{Tm}$ across most of the detector. A set of precision chambers covers the region $|\eta|<2.7$ with three layers of monitored drift tubes, complemented by cathode strip chambers in the forward region, where the background is highest. The muon trigger system covers the range $|\eta|<2.4$ with resistive plate chambers in the barrel and thin gap chambers in the endcap regions.

A two-level trigger system is used to select interesting events [1]. It consists of a hardware-based first-level trigger (Level-1, L1) and a software-based high-level trigger (HLT) running on a farm of approximately $50 \mathrm{k}$ processing units. The L1 trigger decision is formed by the Central Trigger Processor, which receives inputs from the L1 calorimeter (L1Calo) [15] and L1 muon triggers as well as several other subsystems. The L1 trigger decision is formed with a latency of $2.2 \mu \mathrm{s}$. The HLT has access to the full event and a decision is made within an average time of $500 \mathrm{~ms}$.

\section{Description of the $E_{\mathrm{T}}^{\text {miss }}$ trigger algorithms}

The operational demands of the trigger prioritize low latency, rapid processing, and large background rejection while making use of limited detector information. Thus the online $E_{\mathrm{T}}^{\text {miss }}$ trigger algorithms are specifically designed for this purpose and so differ from the offline $E_{\mathrm{T}}^{\text {miss }}$ reconstruction algorithms used in subsequent physics analyses [16, 17].

The ATLAS HLT processes approximately $100 \mathrm{kHz}$ of L1 accepted events, of which about 5 to $10 \mathrm{kHz}$ come from the $\mathrm{L} 1 E_{\mathrm{T}}^{\mathrm{miss}}$ trigger. The HLT $E_{\mathrm{T}}^{\text {miss }}$ algorithms accept events at a rate of about $1200 \mathrm{~Hz}$ averaged over a typical LHC fill [18]. The requirement that the $E_{\mathrm{T}}^{\mathrm{miss}}$ algorithms utilize not more than $\mathcal{O}(100 \mathrm{~ms})$ makes the use of inner-detector tracking information generally too computationally expensive, since the corresponding evaluation time can take $\mathcal{O}(1-5 \mathrm{~s})$. Thus, all of the algorithms described below use only the calorimeter. $^{2}$

For all algorithms the energy measured by the calorimeter is associated with some set of energy depositions, generally referred to as elements. The definition of the set of elements is algorithm-dependent. For example, the set of elements could be all of the calorimeter cells or the reconstructed jets. In each case, the individual elements characterize the local energy deposits, while the complete set captures the overall distribution of energy in the calorimeter. Elements are indexed by the label $i$; the energy $E_{i}$ deposited in each element is also associated with a polar angle $\theta_{i}$ (or equivalently a pseudorapidity $\eta_{i}$ ) and an azimuthal angle $\phi_{i}$.

\footnotetext{
${ }^{2}$ Here and in what follows it should be understood that the singular 'calorimeter' refers to the calorimeter system as a whole.
} 
The components of the missing transverse momentum two-vector $\vec{E}_{\mathrm{T}}^{\mathrm{miss}}$ are calculated from the energy in the elements in the approximation of massless particles

$$
\begin{aligned}
& E_{x}^{\text {miss }}=-\sum_{i=1}^{\mid \text {Elements } \mid} E_{i} \sin \theta_{i} \cos \phi_{i}, \\
& E_{y}^{\text {miss }}=-\sum_{i=1}^{\mid \text {Elements } \mid} E_{i} \sin \theta_{i} \sin \phi_{i},
\end{aligned}
$$

where $\mid$ Elements $\mid$ indicates the number of elements. The magnitude $E_{\mathrm{T}}^{\text {miss }}=$ $\sqrt{\left(E_{x}^{\text {miss }}\right)^{2}+\left(E_{y}^{\text {miss }}\right)^{2}}$ of this two-vector is used in the selection of candidate events for further study. The quantity $E_{\mathrm{T}_{i}}=E_{i} \sin \theta_{i}$ is conventionally known as the transverse energy, and is useful in characterizing events. The total transverse energy in the calorimeter is given by the scalar sum

$$
\Sigma E_{\mathrm{T}}=\sum_{i=1}^{\mid \text {Elements } \mid} E_{i} \sin \theta_{i} .
$$

The algorithms used are presented in the following sections. They differ in how they select the elements which enter into the sums and in how they make corrections to the energies of the elements.

\subsection{Level-1 trigger}

The ATLAS L1 trigger is implemented in firmware running on custom-made electronics [15]. Analogue sums of the input signals from calorimeter cells forming projective towers are digitized, with the granularity in the projective coordinates $\eta$ and $\phi$ being approximately $\Delta \eta \times \Delta \phi=0.1 \times 0.1$ for the detector region $|\eta|<2.5$ and both larger and less regular for $|\eta|>2.5$, as described in ref. [15]. The digitization results in counts that nominally correspond to $1 \mathrm{GeV}$ in $E_{\mathrm{T}}$. A fixed threshold that depends on $\eta$ is then applied per tower: the energy $E_{i}$ of any tower which is below this threshold is set to zero in the subsequent calculations. The threshold is adjusted to provide a fixed occupancy of $0.5-1 \%$ based on data unbiased by a trigger selection. This occupancy threshold is optimized to give an acceptable rate for a trigger that efficiently selects events with $E_{\mathrm{T}}^{\text {miss }}>150 \mathrm{GeV}$. As the LHC luminosity increased, the occupancy tended to grow, leading to higher thresholds as described in ref. [19].

The calorimeter noise thresholds vary from 1 to $9 \mathrm{GeV}$ depending on the pseudorapidity and whether the calorimeter layer is electromagnetic or hadronic. The noise thresholds were periodically reoptimized during the period under study, particularly when the collider parameters, and as a result the pile-up, were varied. In the performance studies that follow, particular attention is paid to three periods during 2017 that have different pileup distributions; these periods are labelled with the symbols $\alpha, \beta$ and $\gamma$. The pile-up at the start of the LHC fill increased from around $\langle\mu\rangle=40$ for period $\alpha$ to $\langle\mu\rangle=60$ for period $\gamma$. The largest changes in threshold occurred for the towers with $4.0<|\eta|<4.9$ in the electromagnetic layer, and the thresholds were 6,7 and $9 \mathrm{GeV}$ for periods $\alpha, \beta$ and $\gamma$ respectively. After the threshold is applied, the towers are summed into larger projective towers which have an approximate granularity of $\Delta \eta \times \Delta \phi=0.2 \times 0.2$ and are referred 
to as jet elements. The $\vec{E}_{\mathrm{T}}^{\mathrm{miss}}$ is then computed by summing the $x$ and $y$ projections of the jet elements using eq. (3.1).

Events that are accepted by the L1 trigger are transferred to the HLT where the $E_{\mathrm{T}}^{\mathrm{miss}}$ is recalculated using one or more of the algorithms described in sections 3.2 to 3.5 .

\subsection{Trigger using calorimeter cell signals (cell)}

The most basic HLT algorithm, cell, determines $\vec{E}_{\mathrm{T}}^{\mathrm{miss}}$ from a sum over the full set of $188 \mathrm{k}$ calorimeter cells to determine $E_{x}$ and $E_{y}$, without adjusting for hadronic vs electromagnetic calibrations or for pile-up corrections. To reduce the effect of noise from electronics and pile-up, only cells satisfying $\left|E_{i}\right|>2 \sigma_{i}$ are included in this sum. Here $\sigma_{i}$ is the expected energy-equivalent noise in cell $i$ described in ref. [13]. Its value is based on expectations for electronic noise and pile-up prior to data taking. Negative energy cells are included because the LAr electronics are designed so that signals from pile-up in later bunch crossings appear as negative energy and so tend to cancel energy deposits from earlier pile-up signals [20]. For the 2015 and 2016 run periods, the noise thresholds were configured for an average number of interactions $\langle\mu\rangle=30$. For 2017 and 2018 they were configured for $\langle\mu\rangle=40$. In addition, the requirement $E_{i}>-5 \sigma_{i}$ is used to protect against spurious large negative cell signals.

\subsection{Trigger using topological clusters of calorimeter cells (tc_lcw)}

The topological clustering [13] of calorimeter cells forms an early stage of many ATLAS reconstruction algorithms. It offers the possibility of identifying clusters as either electromagnetic or hadronic in origin, and thus allows appropriate calibration ('local cell weighting') before using them as inputs for jet reconstruction and calculation of $E_{\mathrm{T}}^{\mathrm{miss}}$.

Topological clusters are formed in a multistage process. First the algorithm identifies calorimeter seed cells each with $\left|E_{i}\right|>4 \sigma_{i}$. All cells neighbouring a seed cell are collected in all three spatial dimensions and added to the cluster. If any of those neighbouring cells satisfy $\left|E_{i}\right|>2 \sigma_{i}$, then their neighbours are collected as well, and the process continues iteratively until no further neighbours satisfying the requirement can be identified. Finally, all neighbouring cells are added to the cluster, regardless of their energy. After this initial cluster formation, an algorithm is run which splits clusters between local signal maxima (again, in three dimensions). The energies of these clusters are corrected for the type of energy deposit after each one has been classified as being either electromagnetic or hadronic in origin.

These energy-calibrated clusters can be used directly in an $E_{\mathrm{T}}^{\mathrm{miss}}$ calculation, which is denoted tc_lcw. These topological clusters also form the inputs to all of the following algorithms.

\subsection{Trigger based on jets (mht)}

In most events of interest, hadronic jets tend to dominate the visible momentum. Since these jets can be calibrated accurately [21], there is good motivation to use them as the basis of an $E_{\mathrm{T}}^{\text {miss }}$ calculation. In addition, the calculation of the $E_{\mathrm{T}}^{\text {miss }}$ from the calorimeter signals described previously includes energy from pile-up, while jets are corrected on-average for pile-up effects. Using only calibrated jets for $E_{\mathrm{T}}^{\text {miss }}$ reconstruction yields a representation that is referred to as mht. 
The mht algorithm calculates $E_{\mathrm{T}}^{\text {miss }}$ from the negative transverse momentum vector sum of all jets above a threshold of $7 \mathrm{GeV}$ before calibration. The HLT jets are reconstructed from calibrated topological clusters (defined in section 3.3) using the anti- $k_{t}$ jet algorithm with a radius parameter of $R=0.4$ [22] implemented in the Fast Jet toolkit [23].

These jets are calibrated in a procedure similar to that used for offline physics analysis [21]. First, the estimated pile-up contribution to jets is removed using the jet-area-based pile-up suppression method [24, 25]. After pile-up subtraction, jets are calibrated using the simulation-based calibration described in ref. [26]. The energy deposits that arise from photons, electrons or hadronically decaying $\tau$-leptons, are included in the jet reconstruction.

\subsection{Trigger implementing local pile-up suppression (pufit)}

The pufit algorithm corrects for pile-up effects on high- $E_{\mathrm{T}}$ calorimeter signals contributing to $E_{\mathrm{T}}^{\text {miss }}$. It employs a pile-up estimate obtained from a fit to lower- $E_{\mathrm{T}}$ signals. It takes as inputs the topological clusters defined in section 3.3 and combines them into $\eta-\phi$ patches that correspond approximately to the size of a jet with $R=0.4$. A fit is then performed which estimates the energy contribution to each patch from pile-up, based on the energy deposited and its spatial fluctuations across the calorimeter. Finally, the pile-up-subtracted patches are used to determine the $E_{\mathrm{T}}^{\text {miss }}$.

The strategy is based on the assumption that high- $E_{\mathrm{T}}$ energy deposits are associated with a hard-scatter collision of interest whereas the low- $E_{\mathrm{T}}$ deposits are the result of pileup. The pufit algorithm proceeds by performing a fit that constrains to zero (within fluctuations) the summed transverse momentum components $E_{x}$ and $E_{y}$ from the pile-up energy deposits. The $E_{\mathrm{T}}^{\text {miss }}$ vector is then determined by summing the $E_{x}$ and $E_{y}$ of the high- $E_{\mathrm{T}}$ deposits after subtracting the estimated pile-up contributions.

The pufit algorithm uses the measured structure of the energy deposition in each event. This contrasts with other approaches such as that of ref. [27] which estimate pileup contributions by defining a median transverse energy density $\langle\rho\rangle$ that is then used in subtracting pile-up from high- $E_{\mathrm{T}}$ deposits. The pufit algorithm is observed to outperform the standard pile-up-density algorithms in the context of the HLT, so these other algorithms are not described further. The full definition of the algorithm, and the event-by-event fit performed, can be found in appendix A.

\section{Offline object and $E_{\mathrm{T}}^{\mathrm{miss}}$ reconstruction}

When defining selections of events for which performance characteristics are desired, standard ATLAS offline algorithms are used to reconstruct and identify electrons, muons, $\tau$-leptons, jets and $b$-tagged jets, as described in appendix B.

The offline $E_{\mathrm{T}}^{\mathrm{miss}}$ is also computed using these reconstructed objects since they tend to have better resolution than individual tracks or clusters in the calorimeter. First, the contributions from high- $p_{\mathrm{T}}$ electrons, photons, $\tau$-leptons and jets are summed, following the procedure described in ref. [16]. To account for the activity from the underlying event, tracks not associated with one of the above objects are also included in the $E_{\mathrm{T}}^{\text {miss }}$ calculation. The $E_{\mathrm{T}}^{\text {miss }}$ definition described above is referred to as 'tight' in the following. 
In some cases, the so-called 'tenacious' offline $E_{\mathrm{T}}^{\text {miss }}$ definition is used in order to make the jet selections less sensitive to pile-up. With this algorithm, jets that have $|\eta|>2.4$ and $p_{\mathrm{T}}<35 \mathrm{GeV}$ are vetoed, along with jets with $p_{\mathrm{T}}<120 \mathrm{GeV}$ that fail the forward jet vertex tagger (JVT) requirement that utilizes jet correlations to reject pile-up jets in a region without a tracking detector [28]. The working point used corresponds to an efficiency of $92 \%$ for hard-scatter jets. Jets with $|\eta|<2.4$ and $p_{\mathrm{T}}$ within $20-40 \mathrm{GeV}$ are used only if they satisfy a JVT requirement that yields an $85 \%$ efficiency for hard-scatter jets. Jets with $p_{\mathrm{T}}$ within $40-60 \mathrm{GeV}$ and $60-120 \mathrm{GeV}$ are used only if they satisfy a similar requirement with an efficiency of $92 \%$ and $97 \%$ respectively.

For all purposes considered in this paper, the offline $E_{\mathrm{T}}^{\mathrm{miss}}$ is computed without any contribution to the visible momentum from any muon(s). This method of computing $E_{\mathrm{T}}^{\text {miss }}$ facilitates comparison with the $E_{\mathrm{T}}^{\text {miss }}$ trigger algorithms which use calorimeter information only.

\section{$5 \quad E_{\mathrm{T}}^{\text {miss }}$ trigger performance}

The figures of merit used to characterize the performance of the $E_{\mathrm{T}}^{\text {miss }}$ trigger algorithm include: CPU time, trigger rate, efficiencies with respect to well-defined references, stability of the efficiencies for several different kinds of events, and the instantaneous luminosity dependence of these characteristics. Depending on the characteristics under study, the L1 and HLT algorithm performances, both individually and when used consecutively, are of interest. Good performance is characterized by a trigger which has stable high efficiency for signal events of interest, and, at the same time, a stable low output rate.

The trigger efficiency is defined by:

$$
\varepsilon\left(\mathcal{S}_{i}\right)=\frac{N\left(\text { trigger } \mid \mathcal{S}_{i}\right)}{N\left(\mathcal{S}_{i}\right)},
$$

where $N\left(\mathcal{S}_{i}\right)$ is the size of the sample of events satisfying some selection $\mathcal{S}_{i}$ which is typically designed to isolate events within a narrow range of $E_{\mathrm{T}}^{\text {miss }}$. To assess the efficiency of the trigger, $\mathcal{S}_{i}$ is relaxed to capture events that satisfy some lower $E_{\mathrm{T}}^{\text {miss }}$ threshold. In either case, the numerator $N\left(\right.$ trigger $\left.\mid \mathcal{S}_{i}\right)$ is the size of the subset of events that also satisfies the $E_{\mathrm{T}}^{\text {miss }}$ trigger requirement.

\subsection{Background model based on detector resolution}

The $E_{\mathrm{T}}^{\text {miss }}$ trigger rate behaviour in the absence of pile-up corrections can be studied with the cell $E_{\mathrm{T}}^{\text {miss }}$ algorithm. This algorithm does not attempt to correct for the effects of pileup, other than via adjustments to the cell noise thresholds. A model has been constructed that captures the dependencies of the unbiased event acceptance (and hence trigger rate) of the cell $E_{\mathrm{T}}^{\text {miss }}$ trigger algorithm on pile-up. The model is sufficient for the purpose of understanding the behaviour of the rate and demonstrates the need for more-sophisticated algorithms to deal with the large increase in pile-up through the period under study. 


\begin{tabular}{|llll|}
\hline Trigger & Random triggers & $L 1 E_{\mathrm{T}}^{\text {miss }}>30 \mathrm{GeV}$ & $L 1 E_{\mathrm{T}}^{\text {miss }}>50 \mathrm{GeV}$ \\
Prescale & $\mathcal{O}\left(10^{6}\right)$ & $\mathcal{O}\left(10^{5}\right)$ & $\mathcal{O}\left(10^{3}\right)$ \\
\hline
\end{tabular}

Table 1. Triggers used for comparing the cell $E_{\mathrm{T}}^{\mathrm{miss}}$ acceptance model with data.

The cell $E_{\mathrm{T}}^{\mathrm{miss}}$ distribution is modelled with two components. The first is due to calorimeter energy resolution effects. This resolution is assumed to depend on the instantaneous number $\mu$ of $p p$ interactions per bunch crossing only through their combined contribution to the total calorimeter transverse energy $\Sigma E_{\mathrm{T}}$, upon which the resolution in turn depends. The second component is the high $E_{\mathrm{T}}^{\text {miss }}$ tail of the distribution, which is assumed to arise from events with rarer measurement fluctuations and events containing non-interacting particles (such as semileptonic decays of $b$ - or $c$-hadrons). The probability of the second class of fluctuations is assumed to scale linearly with instantaneous luminosity. The two components are combined to form the vector sum of the two $E_{\mathrm{T}}^{\text {miss }}$ values, with the azimuthal angle difference between the two components randomly oriented with respect to each other. By modelling the dependencies in this way, and by measuring the parameters of the model at low luminosity (and hence low pile-up), predictions of the $E_{\mathrm{T}}^{\text {miss }}$ distribution and trigger rates can be obtained for higher $\mu$. The detailed description of the model may be found in appendix $\mathrm{C}$.

To compare the calculation with measurements, data are selected by combining events obtained with several triggers, shown in table 1 . For low $E_{\mathrm{T}}^{\text {miss }}$ values, events obtained with an unbiased random trigger (zero bias) are used. The background events, which dominate the rate, were selected using a zero bias trigger, weighted to the instantaneous luminosity per bunch by requiring that an electron trigger fired in the previous LHC orbit of this bunch. Such triggers are prescaled, meaning that only one in $N$ events is accepted for some number $N$. Since the prescale factor $N$ for random triggers is high, there are not enough recorded events at high $E_{\mathrm{T}}^{\text {miss }}$ for the study of the trigger background. These events are therefore supplemented with samples collected by a suite of triggers which require L1 $E_{\mathrm{T}}^{\text {miss }}$ to be greater than a set of thresholds in the range $30 \mathrm{GeV}$ to $50 \mathrm{GeV}$, as shown in table 1 . The efficiencies for selecting events with higher L1 $E_{\mathrm{T}}^{\text {miss }}$ thresholds (and lowest prescales) are found successively from those selected at lower thresholds (and correspondingly higher prescale), until those with the lowest $E_{\mathrm{T}}^{\text {miss }}$ have their efficiencies determined using the random trigger.

Figure 1 compares the two-component model and its individual components with the full $E_{\mathrm{T}}^{\mathrm{miss}}$ distribution measured in data. When comparing data with the model, it is assumed that the instantaneous mean number $\mu$ of interactions per bunch crossing in the model is equal to its time-average $\langle\mu\rangle$ as measured over short periods in data. The data are also expected to have sensitivity to details that are not modelled, such as changes of calorimeter settings and the LHC bunch structure. The lower-luminosity data from earlier years of Run 2 were recorded under conditions different from those for the higherluminosity data recorded in later years, giving differences of up to an order of magnitude in rates depending on threshold and luminosity. As is described in appendix C, the model pa- 


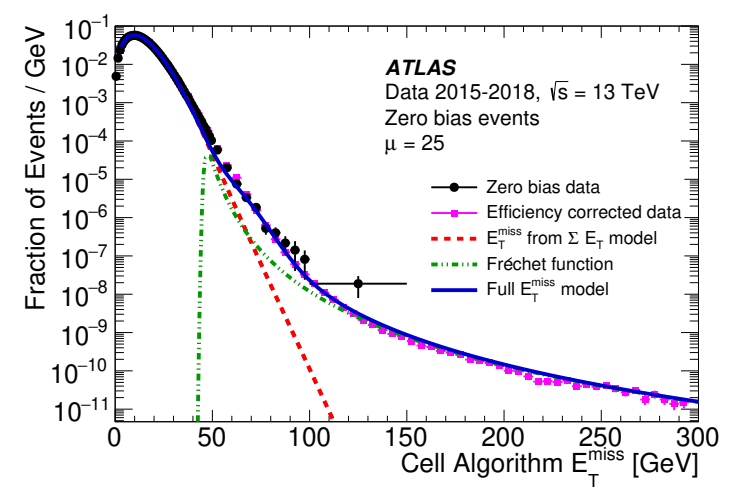

(a)

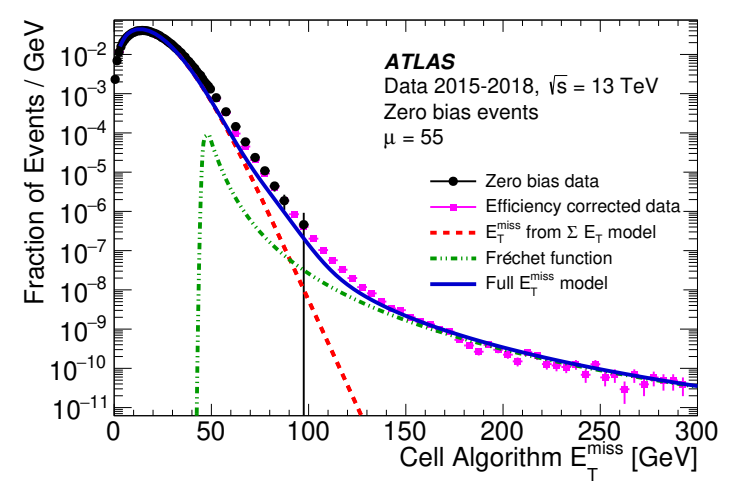

(b)

Figure 1. A comparison of the measured cell $E_{\mathrm{T}}^{\mathrm{miss}}$ distribution with that predicted by the two-component model for two pile-up scenarios compared with data. The circular points show the data collected using zero bias triggers, but have insufficient luminosity to probe the higher $E_{\mathrm{T}}^{\text {miss }}$ portion of the distribution. The square points extend the measured distribution using L1 $E_{\mathrm{T}}^{\text {miss }}>30 \mathrm{GeV}$ and L1 $E_{\mathrm{T}}^{\text {miss }}>50 \mathrm{GeV}$ data. The uncertainties for the data points are statistical only, and much larger for the zero bias data due to the limited luminosity. The dashed (red) curve is the prediction from the calorimeter-resolution part of the model. The dash-dotted (green) curve is the high $E_{\mathrm{T}}^{\text {miss }}$ tail's probability distribution for the mean number of $p p$ interactions $\mu$ in each figure. The solid (blue) curve is the full model prediction computed by combining the $E_{\mathrm{T}}^{\mathrm{miss}}$ from these two individual sources shown in red and green, each calculated for $\mu=\langle\mu\rangle$. The black points show the unbiased $E_{\mathrm{T}}^{\text {miss }}$ distribution measured in data. (a) corresponds to a prediction for $\langle\mu\rangle=25$ while (b) corresponds to $\langle\mu\rangle=55$.

rameters were extracted from the full data set, and therefore are averaged over these effects. Nonetheless, as can be seen in figure 1, the model reproduces the key features of the data over the approximately nine orders of magnitude range of each distribution. Comparisons performed for values of average pile-up in the range $15 \lesssim\langle\mu\rangle \lesssim 60$ show that the model accounts for all qualitative features of the data in this range. Beyond these values it is found to somewhat underestimate (overestimate) the $E_{\mathrm{T}}^{\mathrm{miss}}$ tail for higher (lower) values of $\langle\mu\rangle$.

Three regions can be seen in figure 1 . For low $E_{\mathrm{T}}^{\mathrm{miss}}$, the resolution term dominates, and the rate grows exponentially with increasing $\mu$. At high $E_{\mathrm{T}}^{\text {miss }}$, the tail term dominates, and the rate is linear in $\mu$. Both of these terms contribute at intermediate $E_{\mathrm{T}}^{\text {miss }}$ values. In this region there is a transition from exponential to linear behaviour with increasing $E_{\mathrm{T}}^{\text {miss }}$ threshold. As $\mu$ increases, this transition region moves to higher values of $E_{\mathrm{T}}^{\mathrm{miss}}$. For a fixed $E_{\mathrm{T}}^{\text {miss }}$ threshold trigger, the rate dependence on $\mu$ varies from linear to exponential with increasing $\mu$. The value of $\mu$ at which this transition occurs will vary according to the $E_{\mathrm{T}}^{\mathrm{miss}}$ threshold applied.

Figure 2 shows the prediction for the cell $E_{\mathrm{T}}^{\mathrm{miss}}$ algorithm pass-fraction at fixed threshold as a function of $\mu$. LHC Run-2 luminosities produced instantaneous $\mu$ as high as about 70 , although the figure also shows extrapolated predictions up to $\mu=200$. If the cell $E_{\mathrm{T}}^{\mathrm{miss}}$ algorithm had been the primary $E_{\mathrm{T}}^{\text {miss }}$ trigger during Run 2, the threshold would have been raised considerably to keep the trigger rate within affordable limits. This increase in thresh- 


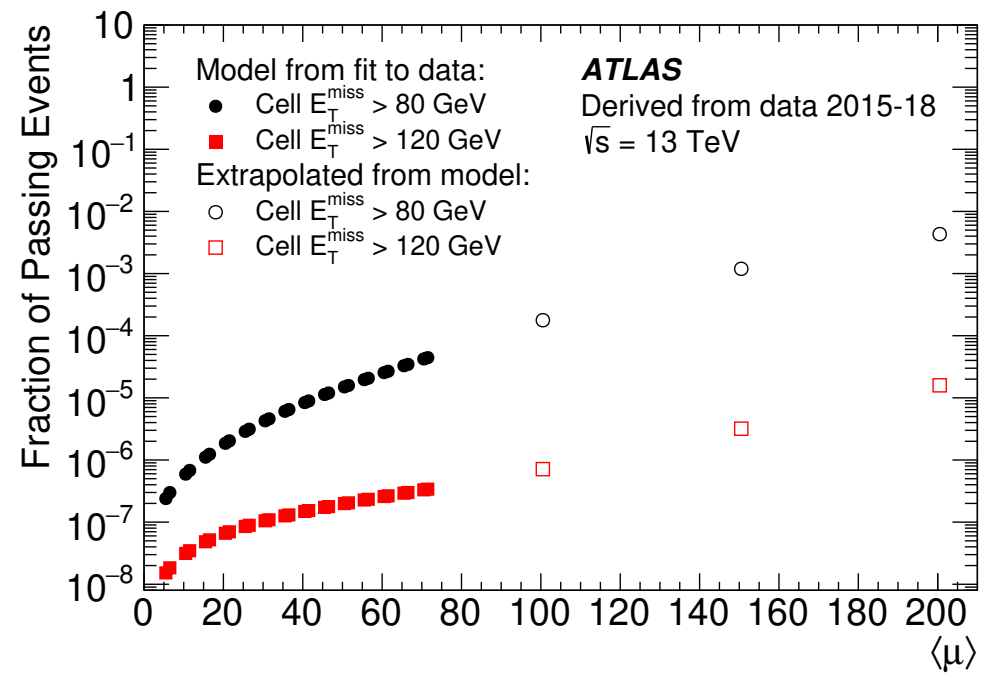

Figure 2. The $E_{\mathrm{T}}^{\text {miss }}$ model predicted trigger rate as a function of $\mu$ for the cell $E_{\mathrm{T}}^{\text {miss }}$ algorithm with a threshold of $80 \mathrm{GeV}$ and $120 \mathrm{GeV}$, assuming no additional pile-up mitigation.

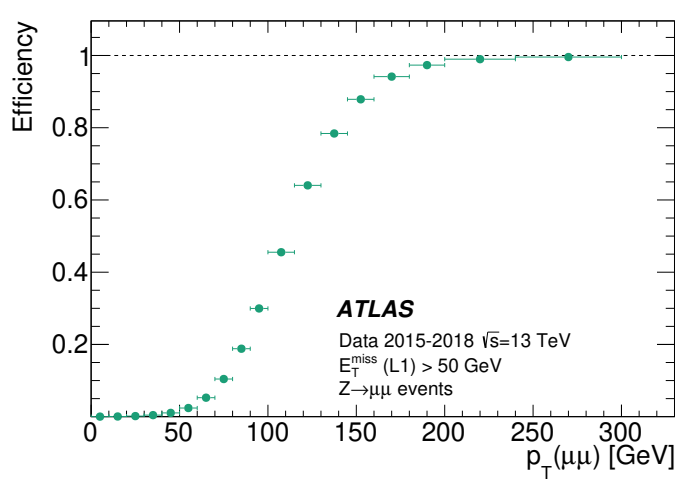

(a)

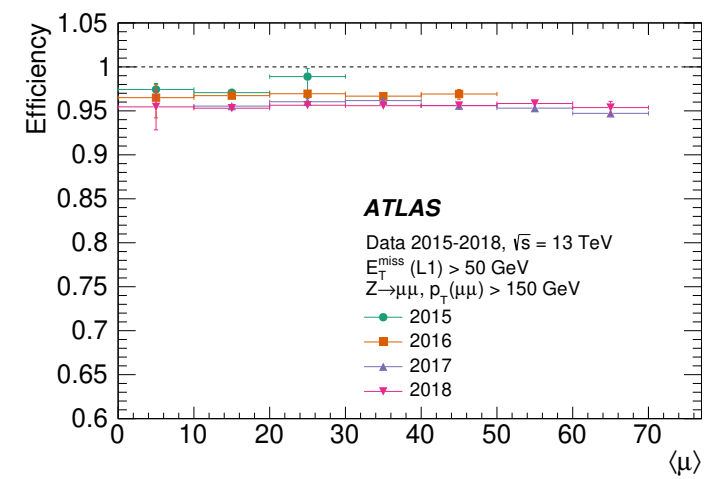

(b)

Figure 3. (a) The L1 $E_{\mathrm{T}}^{\text {miss }}$ trigger efficiency, shown as a function of $p_{\mathrm{T}}(\mu \mu)$ in $Z \rightarrow \mu \mu$ events. (b) The efficiencies in the plot are shown for events satisfying a $Z \rightarrow \mu \mu$ selection and with $p_{\mathrm{T}}(\mu \mu)$ larger than $150 \mathrm{GeV}$ vs pile-up for each of the four years of data taking. The uncertainties are statistical.

old would have significantly decreased the efficiency for signal events. Algorithms which better correct for pile-up were therefore introduced for Run 2 and used either in conjunction with or in place of the cell $E_{\mathrm{T}}^{\mathrm{miss}}$ algorithm.

\subsection{Level-1 trigger performance}

The efficiency of the L1 $E_{\mathrm{T}}^{\text {miss }}$ trigger is determined using a $Z \rightarrow \mu \mu$ events. The muons have little interaction with the calorimeter, so the transverse momentum $p_{\mathrm{T}}(\mu \mu)$ of the dimuon system provides a good estimate of the $E_{\mathrm{T}}^{\text {miss }}$ expected in the trigger calculations.

To select events with two muons, a trigger requiring either two muon candidates each with $p_{\mathrm{T}}>14 \mathrm{GeV}$, or an asymmetric threshold of $22 \mathrm{GeV}$ for the leading muon and $8 \mathrm{GeV}$ 


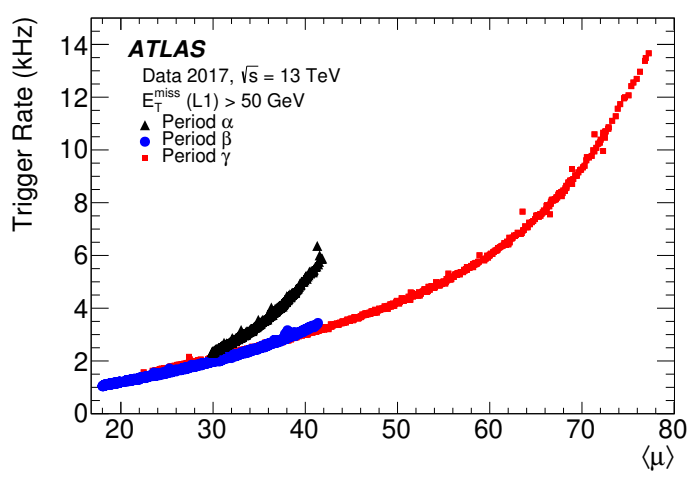

(a)

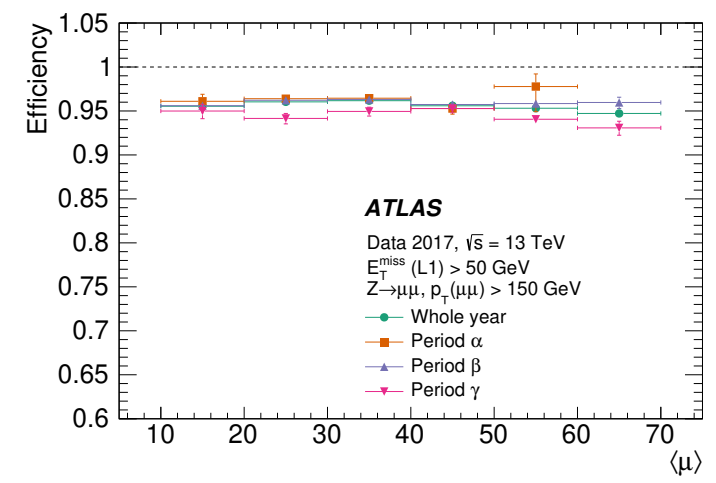

(b)

Figure 4. (a) The L1 $E_{\mathrm{T}}^{\text {miss }}$ trigger rate as a function of $\langle\mu\rangle$ for runs in three different periods $(\alpha$, $\beta, \gamma$ ) in the year 2017. (b) The L1 $E_{\mathrm{T}}^{\text {miss }}$ trigger efficiency is shown as a function of mean pile-up for events satisfying a $Z \rightarrow \mu \mu$ selection and with $p_{\mathrm{T}}(\mu \mu)$ larger than $150 \mathrm{GeV}$ in three periods during the year 2017. The uncertainties are statistical.

for the sub-leading muon was used. The offline selection then requires that each of the two muons has $p_{\mathrm{T}}>25 \mathrm{GeV}$, and that the dimuon invariant mass be in the range $66.6 \mathrm{GeV}<$ $m(\mu \mu)<116.6 \mathrm{GeV}$.

The efficiency is shown as a function of $p_{\mathrm{T}}(\mu \mu)$ in figure 3 a for an L1 nominal threshold of $50 \mathrm{GeV}$, at which the algorithm was generally run without prescaling. It can be observed that the algorithm achieves an efficiency of approximately $90 \%$ for a dimuon $p_{\mathrm{T}}$ of $150 \mathrm{GeV}$. The $\mathrm{L} 1 E_{\mathrm{T}}^{\text {miss }}$ trigger efficiency for a $Z \rightarrow \mu \mu$ selection is shown as a function of $\langle\mu\rangle$ for different years in figure 3b. A threshold of $p_{\mathrm{T}}(\mu \mu)>150 \mathrm{GeV}$ is used for the efficiency calculation since for $E_{\mathrm{T}}^{\text {miss }}$ values in the range $150-175 \mathrm{GeV}$, the L1 trigger is sufficiently close to fully efficient to be interesting for many physics analyses. It is observed that the same efficiency was maintained to within a few percent as the pile-up increased,

Figure 4a shows the corresponding typical trigger rate as a function of the mean pileup $\langle\mu\rangle$, which rises with increasing luminosity. Each of the three periods shown has its own set of values of the L1 calorimeter noise thresholds, which increase with increasing $\langle\mu\rangle$ as the period changes from $\alpha$ to $\beta$ to $\gamma$. The effect of the different noise thresholds used during the periods in 2017 (labelled $\alpha, \beta$ and $\gamma$ ), can be observed. As anticipated, higher calorimeter noise thresholds lead to much reduced trigger rates, particularly at higher $\langle\mu\rangle$.

The L1 $E_{\mathrm{T}}^{\text {miss }}$ trigger efficiency for a $Z \rightarrow \mu \mu$ selection is shown as a function of $\langle\mu\rangle$ is shown for the three periods with different noise thresholds during 2017 in figure 4b. Even though the calorimeter noise thresholds increase to moderate the trigger rate, the efficiency remains stable.

\subsection{High-level trigger performance}

The HLT background acceptance, which is proportional to the trigger rate, is defined as the fraction of events that have $E_{\mathrm{T}}^{\mathrm{miss}}$ computed by the HLT algorithm above a given threshold. 
It is determined using events collected by a dedicated set of L1 triggers, unbiased by the HLT, as described in section 5.1.

The signal efficiency is determined by events collected using the $Z \rightarrow \mu \mu$ selection described in section 5.2. A subsample is selected with an additional requirement that the L1 trigger satisfy $E_{\mathrm{T}}^{\text {miss }}>50 \mathrm{GeV}$, in order to determine the efficiency of the HLT algorithms alone.

Curves of background rejection versus signal efficiency are obtained by varying the HLT trigger threshold. Figure 5 compares such curves for the four $E_{\mathrm{T}}^{\text {miss }}$ algorithms defined in section 3, for different amounts of pile-up. For low pile-up $(\langle\mu\rangle<20)$ the efficiencies at which the tc_lcw and the mht $E_{\mathrm{T}}^{\mathrm{miss}}$ algorithms have equal-efficiency rejection power within a factor of three to that of pufit $E_{\mathrm{T}}^{\text {miss }}$. The cell $E_{\mathrm{T}}^{\text {miss }}$ algorithm has lower corresponding efficiency. As pile-up increases, tc_lcw and mht suffer the most degradation in their performance, whereas the pufit $E_{\mathrm{T}}^{\text {miss }}$ trigger, which was designed to be robust against increasing pile-up, continues to simultaneously achieve good signal efficiency and large background rejection.

By combining different high-level triggers it was found to be possible to further improve the overall HLT performance. The simplest way to achieve this is by demanding that more than one $E_{\mathrm{T}}^{\text {miss }}$ algorithm indicates that the event has high $E_{\mathrm{T}}^{\mathrm{miss}}$. The rationale for such a combination is as follows. The trigger rate of each algorithm for $E_{\mathrm{T}}^{\mathrm{miss}}$ greater than about $50 \mathrm{GeV}$ is typically dominated by contributions from the resolution tails of poorly measured events which often contain little true $E_{\mathrm{T}}^{\text {miss }}$. Since these tails depend on the details of the algorithm, populations of poorly reconstructed events in the high $E_{\mathrm{T}}^{\text {miss }}$ tails differ between algorithms. By contrast, events with large true $E_{\mathrm{T}}^{\text {miss }}$ caused by invisible particles tend to produce a large $E_{\mathrm{T}}^{\text {miss }}$ with all algorithms. Therefore, requiring events to have large $E_{\mathrm{T}}^{\text {miss }}$ in more than one algorithm, with appropriate thresholds for each, can result in reduced trigger rates for a similar overall efficiency.

The joint use of two $E_{\mathrm{T}}^{\text {miss }}$ algorithms was found to be particularly useful when combining the pufit and cell algorithms. Figure 6 shows the relative signal acceptance and background rejection curves of the combined pufit+cell algorithm compared with those of pufit alone or cell alone. With suitable thresholds, the combinations can have a higher rejection at the same efficiency than does either algorithm used alone.

The efficiencies of the cell, pufit and combined pufit + cell algorithms are shown as a function of $p_{\mathrm{T}}(\mu \mu)$ and as a function of the offline $E_{\mathrm{T}}^{\mathrm{miss}}$ in figure 7 . In order to have a fair comparison between the algorithms, each algorithm's trigger threshold has been set such that their background rejections (and hence trigger acceptance rates) are equal. The combined pufit+cell algorithm is again observed to have higher efficiency for signal events throughout the turn-on region than does either of the individual algorithms. The behaviour is consistent regardless of whether the efficiency is calculated as a function of $p_{\mathrm{T}}(\mu \mu)$ or the offline $E_{\mathrm{T}}^{\text {miss }}$ (with muons treated as invisible).

The improved acceptance for physics analyses that results from using the new algorithms can be considerable. Figure 7 shows that the new pufit+cell trigger reaches its plateau efficiency at a $Z$ boson $p_{\mathrm{T}}$ about $25 \mathrm{GeV}$ below that of the cell trigger operating at the same rate. This permits a correspondingly $25 \mathrm{GeV}$ lower offline $E_{\mathrm{T}}^{\mathrm{miss}}$ threshold to 


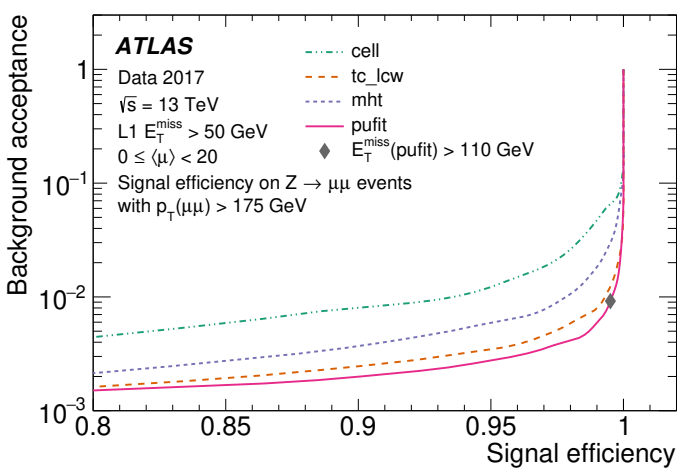

(a)

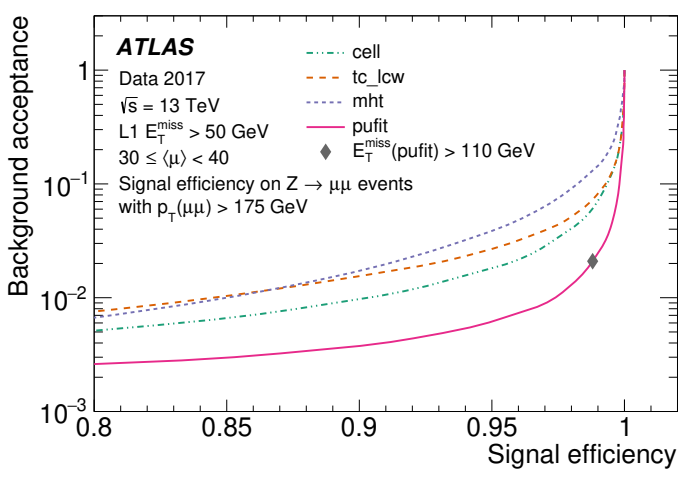

(c)

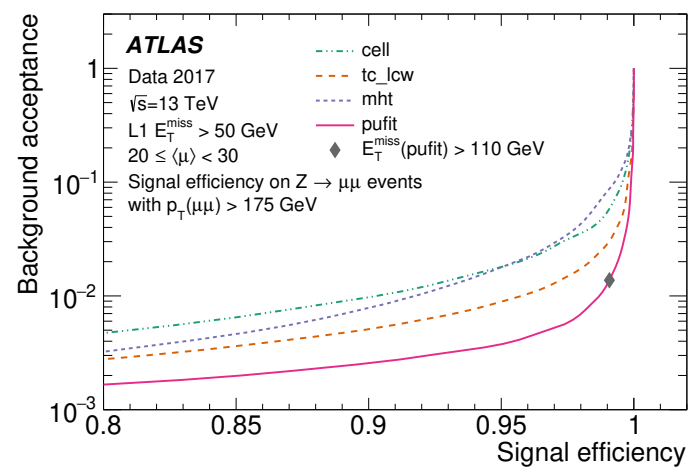

(b)

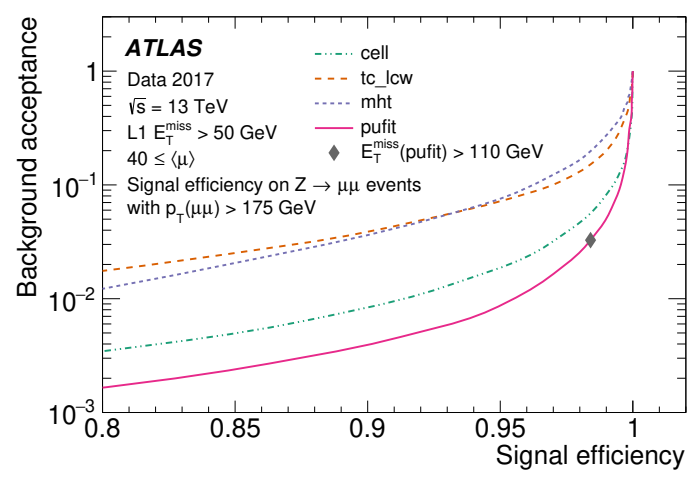

(d)

Figure 5. Background acceptance vs signal efficiency for each of four individual HLT $E_{\mathrm{T}}^{\mathrm{miss}}$ algorithms for a $Z \rightarrow \mu \mu$ selection with $p_{\mathrm{T}}(\mu \mu)>175 \mathrm{GeV}$ for data recorded in the year 2017. The diamond indicates the performance of the pufit $E_{\mathrm{T}}^{\text {miss }}>110 \mathrm{GeV}$ trigger. Each of the four lower panels shows a different range of $\langle\mu\rangle$ : (a) $0 \leq\langle\mu\rangle<20$, (b) $20 \leq\langle\mu\rangle<30$, (c) $30 \leq\langle\mu\rangle<40$ and (d) $40 \leq\langle\mu\rangle$.

be used for any analysis using an $E_{\mathrm{T}}^{\text {miss }}$ trigger. This has a particularly important effect for physics channels in which the $E_{\mathrm{T}}^{\mathrm{miss}}$ distribution falls rapidly. For those analyses that select events on the $E_{\mathrm{T}}^{\text {miss }}$ turn-on region where the trigger is not fully efficient, the pufit+cell algorithm recovers up to double the number of events of interest compared to cell alone. An example is for the search for Higgs to $b$-quarks [11] associated with the decay $Z \rightarrow \nu \nu$. For this analysis the acceptance decreases from $12 \%$ with $E_{\mathrm{T}}^{\text {miss }}>150 \mathrm{GeVto}$ only $5 \%$ with $E_{\mathrm{T}}^{\text {miss }}>200 \mathrm{GeV}$ [29]. If the offline threshold were to increase to $225 \mathrm{GeV}$ the acceptance would have been only $3.5 \%$.

To further examine the efficiency of the trigger algorithms with respect to the offline $E_{\mathrm{T}}^{\text {miss }}$, the $E_{\mathrm{T}}^{\text {miss }}$ trigger efficiency is calculated after applying either an additional offline $E_{\mathrm{T}}^{\text {miss }}>150(175) \mathrm{GeV}$ requirement or an offline $p_{\mathrm{T}}(\mu \mu)>150(175) \mathrm{GeV}$ requirement. Figure 8 (left) shows efficiencies for both the L1 trigger and the full $(\mathrm{L} 1+\mathrm{HLT})$ trigger chain for data recorded at the end of 2018. The trigger efficiencies for a fixed $p_{\mathrm{T}}(\mu \mu)$ 


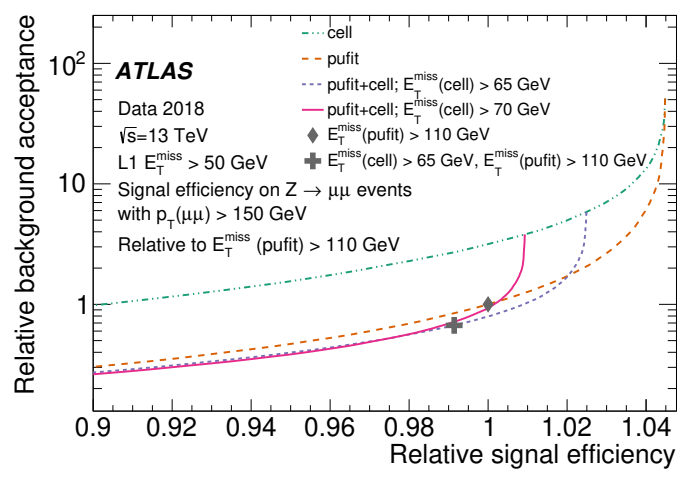

(a)

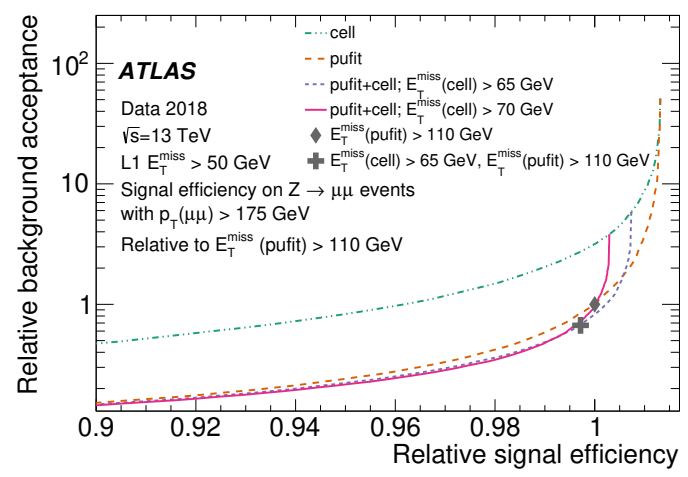

(b)

Figure 6. Relative background acceptance fraction vs. relative efficiency for two different $p_{\mathrm{T}}(\mu \mu)$ thresholds: (a) $p_{\mathrm{T}}(\mu \mu)>150 \mathrm{GeV}$ and (b) $p_{\mathrm{T}}(\mu \mu)>175 \mathrm{GeV}$ for data recorded in the year 2018 . Two of the curves show the performance of the stand-alone cell algorithm and the stand-alone pufit algorithm. The other two show combined algorithms each formed by requiring that the event satisfy both a fixed threshold (either $65 \mathrm{GeV}$ or $70 \mathrm{GeV}$ as shown in the legend) for the cell algorithm and a pufit $E_{\mathrm{T}}^{\text {miss }}$ threshold which varies along the curve. In each plot the background acceptance fractions and the efficiencies are relative to those of the pufit $E_{\mathrm{T}}^{\text {miss }}>110 \mathrm{GeV}$ trigger and thus can be greater than one. The diamond indicates the performance of the pufit $E_{\mathrm{T}}^{\text {miss }}>110 \mathrm{GeV}$ trigger while the cross indicates the performance of the combined (pufit $E_{\mathrm{T}}^{\text {miss }}>110 \mathrm{GeV}$ and cell $E_{\mathrm{T}}^{\text {miss }}>50 \mathrm{GeV}$ ) trigger.

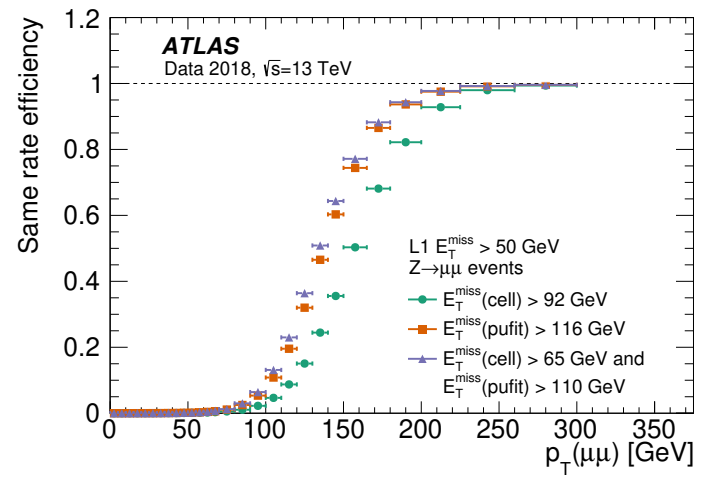

(a)

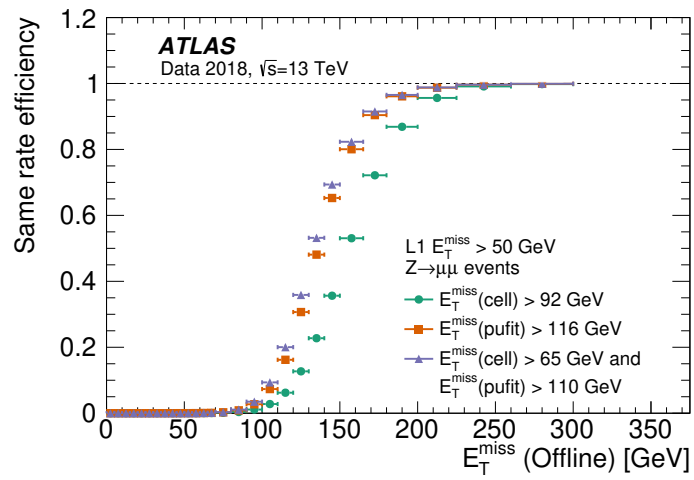

(b)

Figure 7. Turn-on efficiency curves are shown for $Z \rightarrow \mu \mu$ events for three algorithms: the cell algorithm alone, the pufit algorithm alone and the combined cell+pufit algorithm. The thresholds are set such that the algorithms have equal rates, and the data were recorded in the year 2018. (a) The trigger efficiency with respect to $p_{\mathrm{T}}(\mu \mu)$. (b) The trigger efficiency with respect to the offline $E_{\mathrm{T}}^{\text {miss }}$ calculation with muons treated as being invisible. 


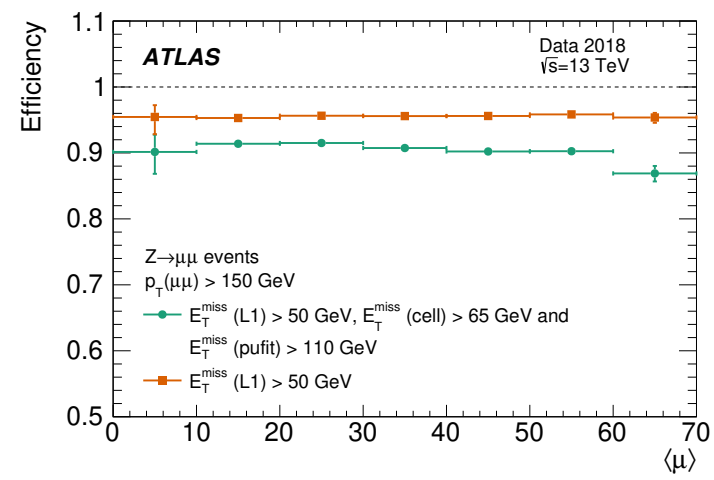

(a)

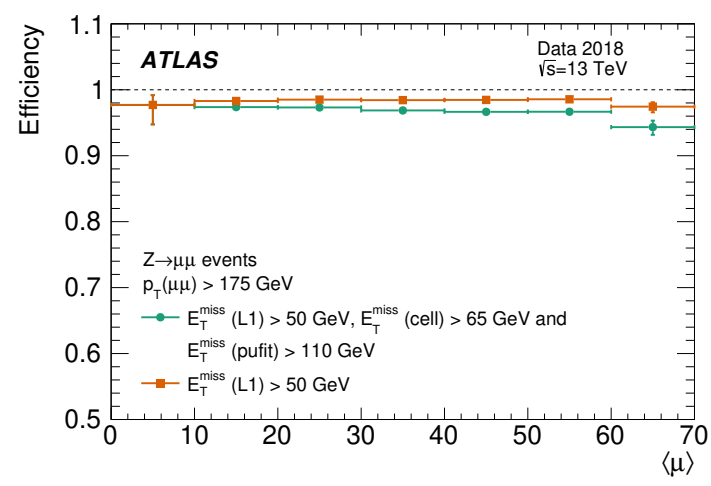

(c)

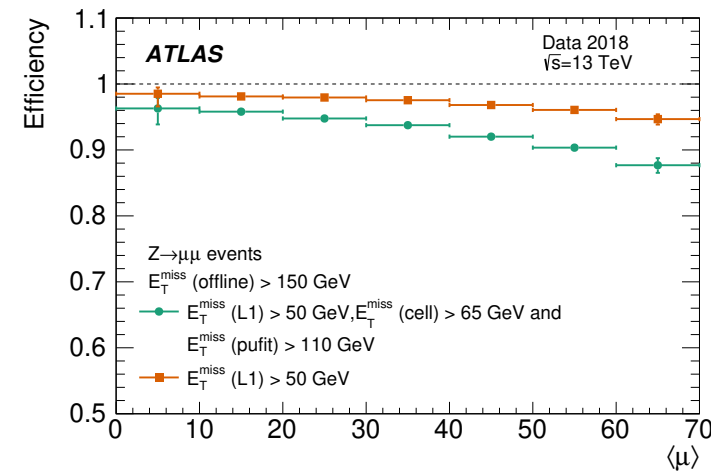

(b)

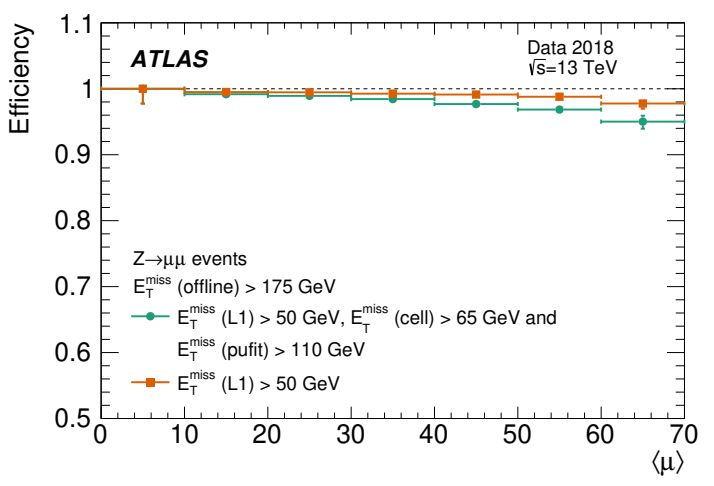

(d)

Figure 8. Efficiencies for $Z \rightarrow \mu \mu$ events are shown for the L1 $E_{\mathrm{T}}^{\text {miss }}>50 \mathrm{GeV}$ trigger (square) and for the complete L1+HLT trigger chain (circle) that also requires pufit $E_{\mathrm{T}}^{\mathrm{miss}}>110 \mathrm{GeV}$. The uncertainties are statistical. Each is shown as a function of $\langle\mu\rangle$, either for a $p_{\mathrm{T}}(\mu \mu)$ threshold as in the left plots: (a) and (c) or for an offline $E_{\mathrm{T}}^{\text {miss }}$ threshold as shown in the right plots: (b) and (d). The upper two plots (a) and (b) show thresholds of $150 \mathrm{GeV}$, while the lower two plots (c) and (d) correspond to thresholds of $175 \mathrm{GeV}$.

threshold show no significant decrease, even for the highest values of $\langle\mu\rangle$. However, when compared with an offline $E_{\mathrm{T}}^{\text {miss }}$ threshold in figure 8 (right), an apparent degradation of the trigger efficiency is observed for high $\langle\mu\rangle$. This indicates that the difference between the online and offline $E_{\mathrm{T}}^{\text {miss }}$ definitions has a larger effect at higher $\langle\mu\rangle$.

\subsection{Trigger menu evolution and performance}

Due to the dependence of the algorithm efficiencies and trigger rates upon luminosity, it was necessary to update the primary physics triggers to cope with the increasing pileup levels. Since the L1 rate was reduced by adjusting calorimeter noise thresholds, only small adjustments needed to be made to the overall L1 threshold. Table 2 summarizes the algorithms and trigger thresholds used during Run-2 data taking. In 2015-2016, the mht $E_{\mathrm{T}}^{\text {miss }}$ was used. From 2016, the pufit $E_{\mathrm{T}}^{\text {miss }}$ was combined with cell $E_{\mathrm{T}}^{\text {miss }}$, thereby mitigating the effect of pile-up. 


\begin{tabular}{|llllcc|}
\hline Year & Trigger name & $\begin{array}{l}\text { HLT algorithm } \\
{[\mathrm{GeV}]}\end{array}$ & $\begin{array}{l}\text { L1 threshold } \\
{[\mathrm{GeV}]}\end{array}$ & $\begin{array}{l}\text { HLT threshold } \\
{[\mathrm{GeV}]}\end{array}$ & $\begin{array}{c}\int \mathcal{L} \mathrm{d} t \\
{\left[\mathrm{fb}^{-1}\right]}\end{array}$ \\
\hline 2015 & HLT_xe70_mht_L1XE50 & mht & 50 & 70 & 3.5 \\
2016 & HLT_xe90_mht_L1XE50 & mht & 50 & 90 & 12.7 \\
2016 & HLT_xe110_mht_L1XE50 & mht & 50 & 110 & 30.0 \\
\hline 2017 & HLT_xe90_pufit_L1XE50 & pufit, cell & 50 & 90,50 & 21.8 \\
2017 & HLT_xe100_pufit_L1XE50 & pufit, cell & 50 & 100,50 & 33.0 \\
2017 & HLT_xe110_pufit_L1XE50(55) & pufit,cell & $50(55)$ & 110,50 & 47.7 \\
\hline 2018 & HLT_xe110_pufit_xe65_L1XE50 & pufit,cell & 50 & 110,65 & 57.0 \\
2018 & HLT_xe110_pufit_xe70_L1XE50 & pufit,cell & 50 & 110,70 & 62.6 \\
\hline
\end{tabular}

Table 2. The evolution of the primary $E_{\mathrm{T}}^{\text {miss }}$ physics triggers through the years of the LHC physics Run 2 from 2015 to 2018. For each year the table shows the algorithms used, the L1 and HLT thresholds applied and the integrated luminosity collected. Where two HLT thresholds are given, the first corresponds to the pufit algorithm and the second to the cell algorithm. In 2017, the pufit algorithm was used in conjunction with an additional requirement that cell $E_{\mathrm{T}}^{\text {miss }}>50 \mathrm{GeV}$, which is not explicit in its name. The integrated luminosities are not exclusive and cannot be summed to obtain a total integrated luminosity.

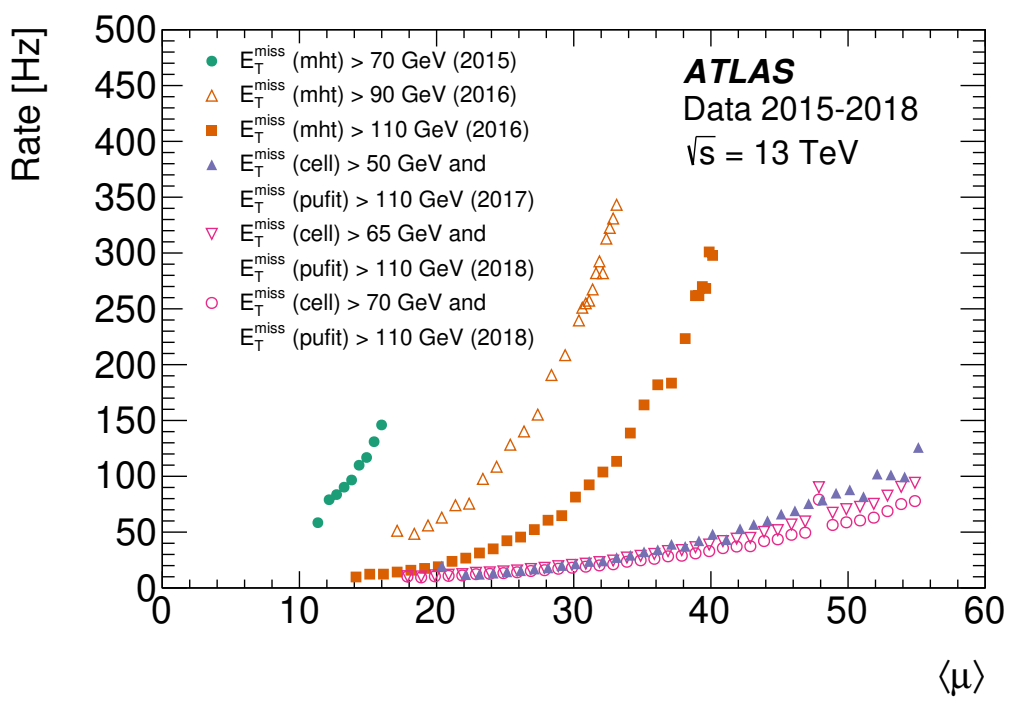

Figure 9. High-level trigger output rates, as a function of $\langle\mu\rangle$, shown separately for example runs in each year 2015-2018, for triggers HLT_xe70_mht (2015), HLT_xe90_mht and HLT_xe110_mht (2016), HLT_xe110_pufit (2017), HLT_xe110_pufit_xe65 and HLT_xe110_pufit_xe70 (2018). The HLT_xe110_pufit trigger used during 2017 also included an implicit requirement of cell $E_{\mathrm{T}}^{\text {miss }}>$ $50 \mathrm{GeV}$. 


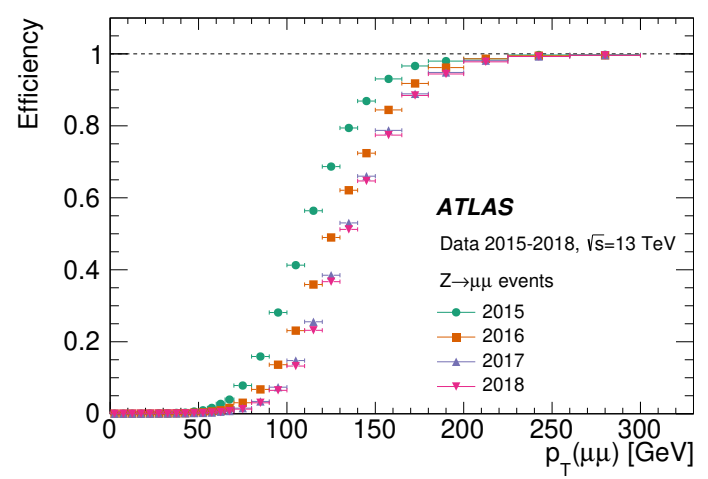

(a)

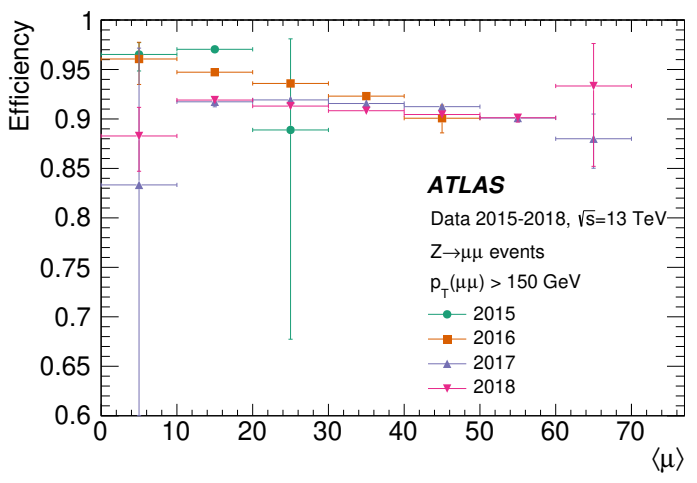

(b)

Figure 10. Full-chain trigger efficiencies for each year (a) as a function of $p_{\mathrm{T}}(\mu \mu)$ and (b) as a function of $\langle\mu\rangle$ for $p_{\mathrm{T}}(\mu \mu)>150 \mathrm{GeV}$. The efficiency corresponds to that of the lowest unprescaled trigger that is adjusted throughout each year (table 2). The uncertainties are statistical.

The trigger names carry information about the algorithms and thresholds used. For example L1_XE50, denotes that the requirement is placed upon the first-level trigger (L1), that the requirement is on the value of $E_{\mathrm{T}}^{\text {miss }}(\mathrm{XE})$, and provides the value of the L1 trigger threshold $(50 \mathrm{GeV})$. The naming convention for full trigger paths can be parsed to give the trigger algorithms and their thresholds. For example, for the trigger path named HLT_xe110_pufit_L1XE50, the prefix HLT indicates that the event must satisfy the highlevel trigger requirement; xe110 indicates that the HLT threshold used was $110 \mathrm{GeV}$; pufit refers to the HLT algorithm used (except in the special case of the cell $E_{\mathrm{T}}^{\text {miss }}$ algorithm, where the additional algorithm name is omitted), and L1XE50 refers to the L1 item used and its threshold.

Typical output rates for various HLT algorithms are shown year-by-year in figure 9 . The reduction in rate obtained by using the pufit-based algorithms is a factor of ten or more for higher values of $\langle\mu\rangle$.

The overall (L1 +HLT) $E_{\mathrm{T}}^{\text {miss }}$ trigger efficiency is shown year-by-year in figure 10 . The efficiency is shown both as a function of $p_{\mathrm{T}}(\mu \mu)$ and as a function of pile-up. The latter demonstrates that the efficiency remained stable within a few percent even at the highest pile-up values recorded.

\subsection{Algorithm computation times}

Average CPU times for the various steps used in the HLT $E_{\mathrm{T}}^{\mathrm{miss}}$ algorithms are given in table 3. For all algorithms except cell, the fraction of the computation time needed for evaluating the final $E_{\mathrm{T}}^{\text {miss }}$ from previously determined input elements is negligible, and most of the CPU time is spent reconstructing cells and topological clusters. All steps satisfy the requirement described in section 3 that the $\mathrm{CPU}$ time does not exceed $\mathcal{O}(100 \mathrm{~ms})$. 


\begin{tabular}{|lccccc|}
\hline \multicolumn{1}{|c}{ Algorithm step } & Time per step $[\mathrm{ms}]$ & \multicolumn{4}{c|}{ Algorithm } \\
& & tc_lcw & pufit & cell & mht \\
\hline Calorimeter cell reconstruction & 20 & $\bullet$ & $\bullet$ & $\bullet$ & $\bullet$ \\
Topological cluster reconstruction & 75 & $\bullet$ & $\bullet$ & - & $\bullet$ \\
Jet reconstruction & 15 & - & - & - & $\bullet$ \\
\hline$E_{\mathrm{T}}^{\text {miss }}$ evaluation time $[\mathrm{ms}]$ & - & - & - & 40 & - \\
\hline Total time $(\mathrm{ms})$ & - & 95 & 95 & 60 & 110 \\
\hline
\end{tabular}

Table 3. The average execution time of each step in computing $E_{\mathrm{T}}^{\text {miss }}$ in the HLT online farm. The $\operatorname{dot}(\bullet)$ indicates the required steps for each algorithm. The time to evaluate the $E_{\mathrm{T}}^{\mathrm{miss}}$ is shown as well, with the total time per step added to the evaluation time.

\subsection{Dependence on event characteristics}

All of the previous efficiencies are computed using the clean reference sample selected with two muons in the final state. This sample is dominated by $Z \rightarrow \mu \mu$ events produced with additional jets. Because the detector response is not identical for events selected according to different criteria, the computation of $E_{\mathrm{T}}^{\text {miss }}$ also depends on the event characteristics, for example whether jets or electrons are required to be present. In this section the trigger efficiency is evaluated and compared for a variety of offline event selections.

To complement the $Z \rightarrow \mu \mu$ events, four other selections are defined, as shown in table 4. The $t \bar{t}$ selections target the pair production of top quarks, and are particularly useful for examining performance in events with a large number of jets. These selections require that the event contain either (i) exactly one electron and no muons or (ii) exactly one muon and no electrons or (iii) exactly one electron and one muon. The vector boson fusion (VBF) selection targets events characterized by two energetic jets, where typically at least one jet is in the forward calorimeter. The selection requires two jets separated by a large pseudorapidity difference and not back-to-back in azimuth. The $W$ boson sample targets the $W \rightarrow e \nu$ process, and samples events with electromagnetic energy deposits that can be larger than $50 \mathrm{GeV}$.

\begin{tabular}{|llll|}
\hline Physics process & Offline $E_{\mathrm{T}}^{\text {miss }}$ definition & Lepton(s) & Kinematics \\
\hline$Z \rightarrow \mu \mu$ & $p_{\mathrm{T}}(\mu \mu)$ & $\mu \mu$ & $66.6<m(\mu \mu)<116.6 \mathrm{GeV}$ \\
$W \rightarrow e \nu$ & Tight & $e$ & - \\
VBF & Tight & $\mu, p_{\mathrm{T}}>30 \mathrm{GeV}$ & Exactly two jets, $p_{\mathrm{T}}>80(50) \mathrm{GeV}$, \\
& & & $|\Delta \phi(\mathrm{jj})|<1.8,|\Delta \eta(\mathrm{jj})|>4.9$ \\
$t \bar{t}$ & Tight & $e, \mu, e^{ \pm} \mu^{\mp}$ & $\geq 2 b$-tagged jets \\
\hline
\end{tabular}

Table 4. Definition of offline analysis selections used for efficiency measurements, labelled by the physics process being examined. All indicated lepton requirements implicitly require $p_{\mathrm{T}}(\ell)>25 \mathrm{GeV}$ unless specified. The offline $E_{\mathrm{T}}^{\text {miss }}$ definitions correspond to different working points. When multiple jets are required with different $p_{\mathrm{T}}$ thresholds, the threshold for the subleading jet is listed in parentheses, e.g. 80 (50) GeV. 


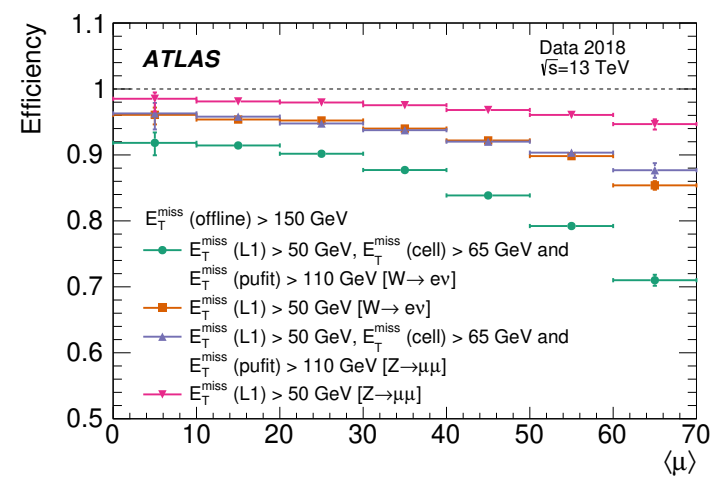

(a)

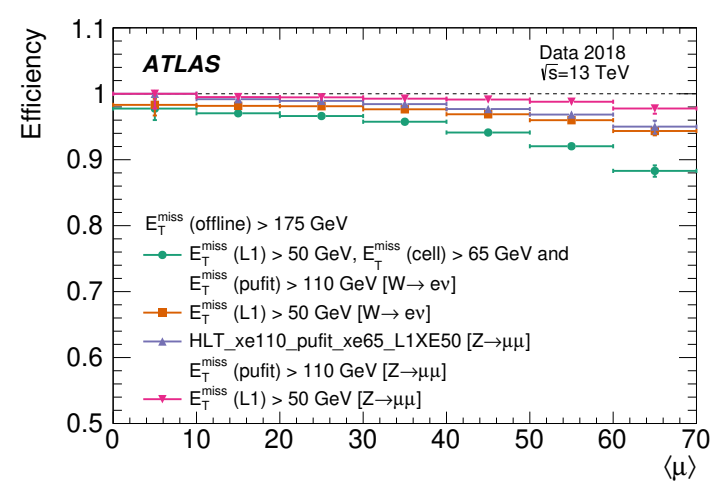

(c)

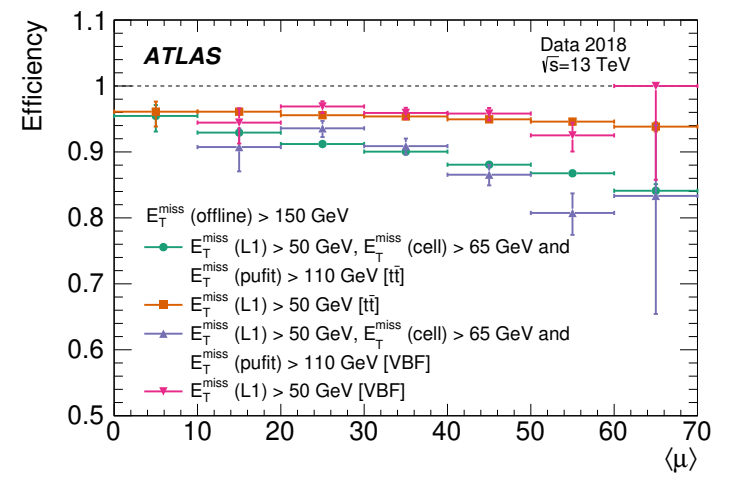

(b)

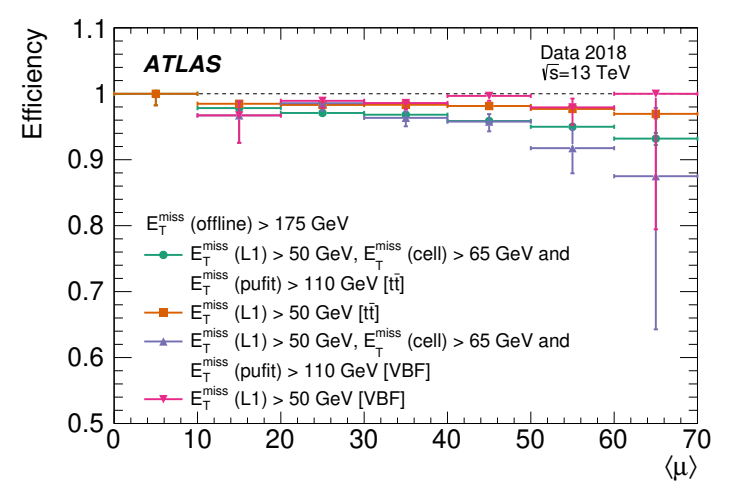

(d)

Figure 11. Efficiencies for the first-level trigger L1XE50 and the combined L1+HLT trigger chain HLT_xe110_pufit_xe65_L1XE50 in data recorded in the year 2018 are shown as a function of $\langle\mu\rangle$ for two different offline $E_{\mathrm{T}}^{\text {miss }}$ thresholds and four different physics selections: (a) $W \rightarrow e \nu$ and $Z \rightarrow \mu \mu$ selections with offline $E_{\mathrm{T}}^{\text {miss }}>150 \mathrm{GeV}$ (b) $t \bar{t}$ and vector boson fusion selections with offline $E_{\mathrm{T}}^{\text {miss }}$ $>150 \mathrm{GeV}$ (c) $W \rightarrow e \nu$ and $Z \rightarrow \mu \mu$ selections with offline $E_{\mathrm{T}}^{\mathrm{miss}}>175 \mathrm{GeV}$ (d) $t \bar{t}$ and vector boson fusion selections with offline $E_{\mathrm{T}}^{\text {miss }}>175 \mathrm{GeV}$. The uncertainties are statistical.

The $W$ boson, $\mathrm{VBF}$ and $t \bar{t}$ samples were each collected using a trigger that selects events containing a single isolated electron or muon. The required lepton transverse momentum thresholds were in the range $24-26 \mathrm{GeV}$, where the $p_{\mathrm{T}}$ value corresponds to the lowest threshold single lepton trigger available for a given luminosity.

Figure 11 shows the stability of the efficiencies with respect to pile-up after requiring that the offline $E_{\mathrm{T}}^{\text {miss }}$ be larger than $150(175) \mathrm{GeV}$ for these four different physics selections. In general, the efficiency for $\langle\mu\rangle>50$ tends to be approximately $10-20 \%$ lower for events containing an electron rather than a muon. A difference in behaviour is not unexpected given that electrons are included in the calculation of the visible momentum, whereas muons are not. The offline $E_{\mathrm{T}}^{\text {miss }}$ calculation uses offline electrons that have better resolution compared to the trigger algorithm. It can also be seen that events containing forward jets in the VBF selections, or containing jets from top quark decays (right) have a somewhat different behaviour than do those events selected without jet requirements (left). Such variations 
are also to be expected, since the $E_{\mathrm{T}}^{\text {miss }}$ resolution and scale change if any jets are present and depend on their energies and the region(s) of the calorimeter in which they are found.

\subsection{Comparison with Monte Carlo simulation}

It is important for most physics analyses to quantify the efficiency with which the trigger selects the events of interest. Depending on the details of the analysis, the $E_{\mathrm{T}}^{\text {miss }}$ trigger efficiency may be determined from data alone, from a Monte Carlo simulation, or from a combination of the two. A concern with using Monte Carlo simulation to derive the $E_{\mathrm{T}}^{\text {miss }}$ trigger efficiency is the effect of any residual difference between data and simulation. For example, as described in section 3.1, the noise thresholds of the L1 $E_{\mathrm{T}}^{\text {miss }}$ trigger algorithm are adjusted periodically during data taking, but it is generally impractical to include such changes in the simulation. Because the $E_{\mathrm{T}}^{\text {miss }}$ triggers use information from the full calorimeter, the efficiency determined using $E_{\mathrm{T}}^{\text {miss }}$ triggers is more sensitive to changes in noise thresholds than the efficiency of other triggers.

Many ATLAS physics analyses render residual $E_{\mathrm{T}}^{\text {miss }}$ trigger inefficiencies largely immaterial by requiring that the offline $E_{\mathrm{T}}^{\text {miss }}$ be larger than $200 \mathrm{GeV}$. This requirement means that selected events are in a region in which the trigger efficiency is greater than $99 \%$ and therefore inefficiencies are negligible. However, some analyses, particularly those in which the number of events falls rapidly with increasing $E_{\mathrm{T}}^{\text {miss }}$ (such as those in refs. [4, 8, 11]) motivate the use of $E_{\mathrm{T}}^{\mathrm{miss}}$ thresholds below the trigger plateau in order to maintain high signal efficiency. For these and similar cases the $E_{\mathrm{T}}^{\text {miss }}$ trigger efficiency needs to be determined, often by using Monte Carlo simulations. Any differences between the simulation and the data may therefore lead to an incorrect calculation of the efficiency if the simulation alone were to be relied upon.

To account for residual differences between data and simulation, corrections referred to as scale factors are determined by measuring the ratio of the trigger efficiency using data to that expected from simulation. These are subsequently applied to correct the signal and background simulation. In the case of the $E_{\mathrm{T}}^{\text {miss }}$ trigger the values of the scale factors vary with properties that include, e.g., the value of the trigger threshold, the cell noise thresholds, the definition of offline $E_{\mathrm{T}}^{\text {miss }}$ and the details of the offline selection. Given that the $E_{\mathrm{T}}^{\text {miss }}$ trigger is used for a large range of offline selections with widely varying final states, no single scale factor suitable for all cases can be found. Instead, analysis-specific corrections must be employed.

Comparisons between the trigger efficiency as predicted by Monte Carlo simulation and as measured in data were performed for both the L1 and the combined L1+HLT trigger chain, using data recorded during 2018. The trigger employed for those data is the combined pufit + cell algorithm with thresholds as indicated in table 2 . The variant of the offline $E_{\mathrm{T}}^{\text {miss }}$ used is that referred to as 'tenacious', and is described in section 4 . The efficiency is measured using events containing a single muon, which, as elsewhere, is treated as being invisible in the $E_{\mathrm{T}}^{\text {miss }}$ calculation. The selection requirements are otherwise similar to the row labelled 'VBF' in table 4, except that: the requirements on the VBF jets are changed such that $|\Delta \phi(\mathrm{jj})|<2.0$ and $m(\mathrm{jj})>200 \mathrm{GeV}$. In addition, for figure 12a, exactly two jets are required and $|\Delta \eta(\mathrm{jj})|>5.0$, while figure $12 \mathrm{~b}$ is binned in jet multiplicity and $|\Delta \eta(\mathrm{jj})|>$ 3.5. This selection is similar to that used in the VBF Higgs-to-invisible analysis [4]. 


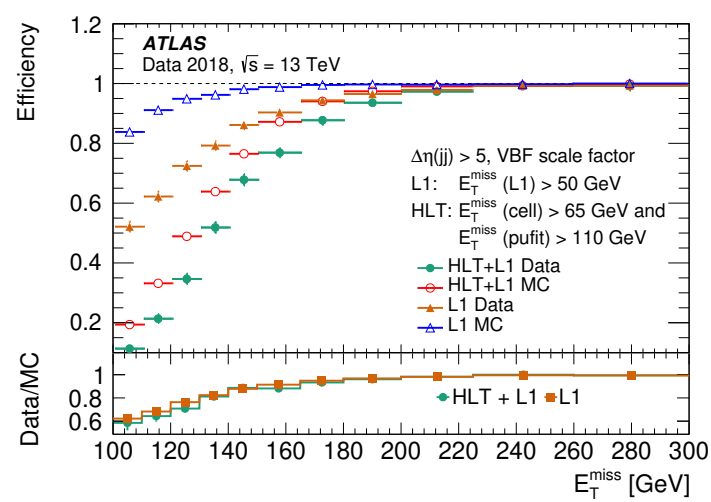

(a)

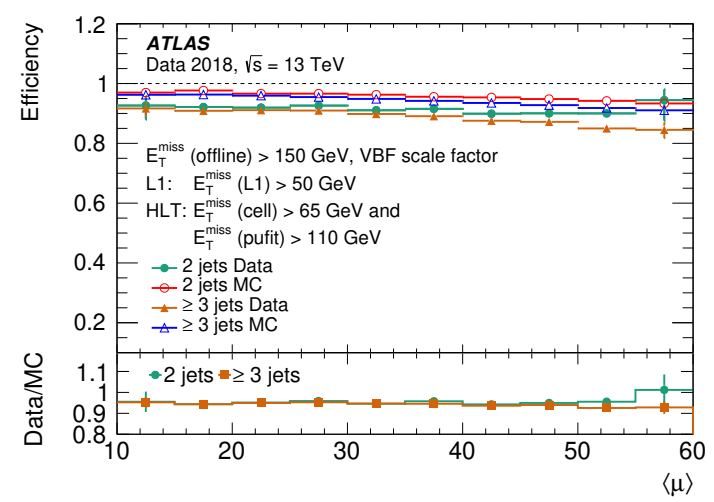

(b)

Figure 12. Efficiencies from data and from simulation for L1 (L1XE50) and the combined L1+HLT chain (HLT_xe110_putfit_xe65_L1XE50) triggers are shown for a VBF selection which requires at least two jets that are well separated in rapidity. The uncertainties are statistical only. The lower panels show the ratios between efficiencies in data and simulation. (a) Efficiencies as a function of the offline $E_{\mathrm{T}}^{\text {miss }}$. In this plot the jet rapidity difference requirement has been tightened to $\Delta \eta(\mathrm{jj})>5$ (b) efficiencies as a function of $\langle\mu\rangle$ for an offline requirement of $E_{\mathrm{T}}^{\text {miss }}>150 \mathrm{GeV}$ using the 'tenacious' working point; the efficiencies in data and MC simulation are compared for two different selections, one requiring exactly two and the other $\geq 3$ jets.

Monte Carlo simulated samples of the $Z \rightarrow \nu \nu, W \rightarrow \ell \nu$ and $Z \rightarrow \ell \ell$ processes were generated at next-to-leading order (NLO) in strong coupling constant $\alpha_{\mathrm{s}}$ using Sherpa 2.2.1 [30]. These calculations use the Comix [31] and OpenLoops [32] matrix element generators, and merging was done with the SHERPA parton shower [33] using the ME+PS@NLO prescription [34]. The NNPDF3.0 parton distribution function (PDF) set [35] computed at next-to-next-to-leading order in $\alpha_{\mathrm{s}}$ was used, along with dedicated parton shower tuning parameters developed for SHERPA 2.2.1 [36]. After the events were generated, the response of the detector [37] is simulated using GEant4 [38]. Each $W$ or $Z$ boson event was overlaid with 15-70 pile-up collisions to match the distribution in data. The pile-up events were simulated using PYтнia 8.1 [39] with the MSTW2008 PDF set [35] and A3 set of parameters tuned to data [40,41]. The efficiency as a function of offline $E_{\mathrm{T}}^{\text {miss }}$ is shown in figure $12 \mathrm{a}$. In the trigger turn-on region at lower values of offline $E_{\mathrm{T}}^{\mathrm{miss}}$, a difference can be observed between data and simulation, an effect largely attributable to the L1 trigger. The lower panel shows the ratio of data-determined to simulated efficiency as a function of the offline $E_{\mathrm{T}}^{\text {miss }}$. This ratio is an example of the scale factor that can be applied to simulated Monte Carlo events to correct their $E_{\mathrm{T}}^{\text {miss }}$ trigger efficiency. Figure $12 \mathrm{~b}$ shows the efficiency with respect to $\langle\mu\rangle$ for a selection that requires offline $E_{\mathrm{T}}^{\mathrm{miss}}>150 \mathrm{GeV}$ and either exactly 2 or $\geq 3$ jets. It can be seen that the Monte Carlo simulations overestimate the efficiency by a few percent. Such considerations show the need both to correct for the differences between data and simulation when working in the turn-on region, and to understand the behaviour of the resulting scale factors for appropriate selections. 


\section{Conclusion}

Despite the considerable increase in luminosity during Run 2 of the LHC (2015-2018), it was possible to maintain the excellent performance of the ATLAS $E_{\mathrm{T}}^{\text {miss }}$ trigger. This was achieved through a dedicated programme of developing, testing, evaluating and optimizing various pile-up mitigation algorithms.

For triggers without any pile-up correction, a steep increase in trigger rate with pileup is observed. This behaviour is consistent with expectations from a two-component background $E_{\mathrm{T}}^{\mathrm{miss}}$ distribution model.

Several $E_{\mathrm{T}}^{\text {miss }}$ trigger algorithms were introduced in ATLAS during LHC Run 2. Both the first-level and high-level trigger algorithms were improved to maintain a similar level of efficiency throughout the data-taking period. These included a new high-level trigger algorithm which uses a fit to determine pile-up-induced local energy deposits in individual events to reduce the impact of increasing luminosity on the $E_{\mathrm{T}}^{\text {miss }}$ trigger rate. In addition, it was found that combining algorithms related to different sources of high- $E_{\mathrm{T}}^{\mathrm{miss}}$ tails could, with an appropriate choice of thresholds, help maintain high efficiency while keeping trigger rates under control. The new algorithms enabled the offline $E_{\mathrm{T}}^{\text {miss }}$ selection to become efficient about $25 \mathrm{GeV}$ earlier than would have previously been possible. In the trigger turn-on region the new algorithms recovered up to double the number of events that would have been possible previously.

A study of the $E_{\mathrm{T}}^{\text {miss }}$ trigger performance for different signal samples shows only a small degradation of the efficiency despite the factor of four increase in instantaneous luminosity during the four-year LHC Run-2 period. The $E_{\mathrm{T}}^{\text {miss }}$ trigger behaviour agrees in general with predictions from Monte Carlo simulations. However, the low- $E_{\mathrm{T}}^{\text {miss }}$ region, where the trigger is not fully efficient, is more difficult to model precisely. Since different $E_{\mathrm{T}}^{\text {miss }}$ algorithms respond differently depending on the characteristics of the event, it is necessary for analyses working in this low- $E_{\mathrm{T}}^{\text {miss }}$ region to determine the specific corrections appropriate to their particular event selection.

The predictions from the background model extend up to $\mu=200$, the value anticipated for the HL-LHC [29]. The predicted rates are those that would be obtained if no changes were made to the algorithm to mitigate pile-up. The equal-threshold acceptance fractions predicted for this level of pile-up are about two orders of magnitude higher than in Run 2, making that environment even more challenging for the $E_{\mathrm{T}}^{\text {miss }}$ trigger. The upgrades planned for ATLAS from 2021 to 2025 will enable a factor of ten increase in trigger rate and a first-level trigger that takes advantage of the full calorimeter granularity. As found in the results presented in this paper, mitigation of pile-up is possible, by use of specificallydesigned algorithms which can achieve lower rates for the same signal efficiency. Use of tracking information can also mitigate the pile-up effect, as can increasing the cell noise thresholds. However, from the rate predictions it is clear that further development of the high-level $E_{\mathrm{T}}^{\text {miss }}$ trigger algorithms will also be required. 


\section{Acknowledgments}

We thank CERN for the very successful operation of the LHC, as well as the support staff from our institutions without whom ATLAS could not be operated efficiently.

We acknowledge the support of ANPCyT, Argentina; YerPhI, Armenia; ARC, Australia; BMWFW and FWF, Austria; ANAS, Azerbaijan; SSTC, Belarus; CNPq and FAPESP, Brazil; NSERC, NRC and CFI, Canada; CERN; CONICYT, Chile; CAS, MOST and NSFC, China; COLCIENCIAS, Colombia; MSMT CR, MPO CR and VSC CR, Czech Republic; DNRF and DNSRC, Denmark; IN2P3-CNRS and CEA-DRF/IRFU, France; SRNSFG, Georgia; BMBF, HGF and MPG, Germany; GSRT, Greece; RGC and Hong Kong SAR, China; ISF and Benoziyo Center, Israel; INFN, Italy; MEXT and JSPS, Japan; CNRST, Morocco; NWO, Netherlands; RCN, Norway; MNiSW and NCN, Poland; FCT, Portugal; MNE/IFA, Romania; MES of Russia and NRC KI, Russia Federation; JINR; MESTD, Serbia; MSSR, Slovakia; ARRS and MIZŠ, Slovenia; DST/NRF, South Africa; MINECO, Spain; SRC and Wallenberg Foundation, Sweden; SERI, SNSF and Cantons of Bern and Geneva, Switzerland; MOST, Taiwan; TAEK, Turkey; STFC, United Kingdom; DOE and NSF, United States of America. In addition, individual groups and members have received support from BCKDF, CANARIE, Compute Canada and CRC, Canada; ERC, ERDF, Horizon 2020, Marie Skłodowska-Curie Actions and COST, European Union; Investissements d'Avenir Labex, Investissements d'Avenir Idex and ANR, France; DFG and AvH Foundation, Germany; Herakleitos, Thales and Aristeia programmes co-financed by EU-ESF and the Greek NSRF, Greece; BSF-NSF and GIF, Israel; CERCA Programme Generalitat de Catalunya and PROMETEO Programme Generalitat Valenciana, Spain; Göran Gustafssons Stiftelse, Sweden; The Royal Society and Leverhulme Trust, United Kingdom.

The crucial computing support from all WLCG partners is acknowledged gratefully, in particular from CERN, the ATLAS Tier-1 facilities at TRIUMF (Canada), NDGF (Denmark, Norway, Sweden), CC-IN2P3 (France), KIT/GridKA (Germany), INFN-CNAF (Italy), NL-T1 (Netherlands), PIC (Spain), ASGC (Taiwan), RAL (U.K.) and BNL (U.S.A.), the Tier-2 facilities worldwide and large non-WLCG resource providers. Major contributors of computing resources are listed in ref. [50].

\section{A Full definition of the trigger implementing local pile-up suppression}

The details of the pufit algorithm, introduced in section 3.5, are as follows.

To estimate the contribution from high- $E_{\mathrm{T}}$ energy deposits, the calorimeter is divided into equal area 'patches'. Using 8 divisions in $\phi$ and 14 in $\eta$ yields a total of 112 patches, each with an area similar to that of an $R=0.4$ jet. The energy for each patch is computed from the sum of all the clusters that fall within that patch. To prevent a jet from being split into two low- $E_{\mathrm{T}}$ patches, four sets of patches are constructed in parallel, with each set shifted by half of a patch size along $\eta$ and/or $\phi$. From the four candidate sets of patches, the algorithm selects the set that yields the largest scalar sum of $E_{\mathrm{T}}$ from the high- $E_{\mathrm{T}}$ patches. The transverse energy in each patch is then used to select the hard-scatter and 
pile-up patches. Finally, a fit is performed to determine the pile-up contribution to each hard-scatter patch.

The fit is constrained by two assumptions: first that the vector sum of $\vec{E}_{\mathrm{T}}^{\text {miss }}$ over all pile-up contributions should be zero, and second that the pile-up should be relatively evenly distributed throughout the detector.

Stage 1: selection of hard-scatter patches. The hard-scatter patch threshold is determined for each event from the trimmed mean and variance of the distribution of the patch $E_{\mathrm{T}}$ values in the event. The patch $E_{\mathrm{T}}$ values are sorted in ascending order and a trimmed mean is determined by discarding the $5 \%$ of patches with the lowest $E_{\mathrm{T}}$ and the $5 \%$ of patches with the highest $E_{\mathrm{T}}$. The trimmed mean is given by:

$$
\left\langle E_{\mathrm{T}}\right\rangle_{\mathrm{patch}}=\frac{1}{N} \sum_{j=0.05 N}^{0.95 N} E_{\mathrm{T}_{j}}
$$

where $N$ is the total number of patches. The variance of the patch $E_{\mathrm{T}}$ values is estimated using the sample variance, calculated as

$$
V_{\mathrm{patch}}=\frac{1}{N}\left\{\sum_{j=1}^{0.95 N}\left(E_{\mathrm{T}_{j}}-\left\langle E_{\mathrm{T}}\right\rangle_{\mathrm{patch}}\right)^{2}+\sum_{j=1}^{0.05 N}\left(E_{\mathrm{T}_{j}}-\left\langle E_{\mathrm{T}}\right\rangle_{\mathrm{patch}}\right)^{2}\right\},
$$

where the second term uses the lowest $5 \%$ of patches (already included in the first term) to estimate the contribution to the variance from pile-up in the highest $5 \%$. The highest $5 \%$ are excluded because the sample is biased by hard-scatter jets. The patch threshold is then set to

$$
E_{\mathrm{T}}^{\text {thres }}=\left\langle E_{\mathrm{T}}\right\rangle_{\mathrm{patch}}+n_{\sigma} \sqrt{V_{\mathrm{patch}}},
$$

with $n_{\sigma}$ chosen to be 5 , based on rate considerations. The typical value for $E_{\mathrm{T}}^{\text {thres }}$ after the $\mathrm{L} 1$ requirement is $30 \mathrm{GeV}$.

With the threshold determined, patches are categorized as high- $E_{\mathrm{T}}$ or low- $E_{\mathrm{T}}$ if they have transverse energy above or below $E_{\mathrm{T}}^{\text {thres }}$, respectively. Any event that has no patch above threshold has pufit $E_{\mathrm{T}}^{\mathrm{miss}}$ set to zero and is rejected by the trigger. Approximately $97 \%$ of background events that pass the L1XE50 trigger have at least one hard-scatter patch.

Stage 2: performing the fit. The fit of the pile-up contribution to each of the high- $E_{\mathrm{T}}$ patches is performed by minimizing the $\chi^{2}$ function

$$
\chi^{2}\left(\mathcal{E}_{\mathrm{T}_{1}}, \ldots, \mathcal{E}_{\mathrm{T}_{m}}\right)=\Delta^{T} V^{-1} \Delta
$$

In this expression $V$ is the associated covariance matrix, and the variables $\mathcal{E}_{\mathrm{T}_{k}}$ are the pile-up contributions to the transverse energies of the $m$ high- $E_{\mathrm{T}}$ patches. The values of $\mathcal{E}_{\mathrm{T}_{k}}$ are determined by minimizing this $\chi^{2}$ function. The $(m+2)$-dimensional vector $\Delta$ is 
given by

$$
\Delta=\left(\begin{array}{c}
\sum_{i=1}^{N_{\text {low }}} E_{x_{i}}+\sum_{k=1}^{m} \mathcal{E}_{\mathrm{T}_{k}} \cos \phi_{k} \\
\sum_{i=1}^{N_{\text {low }}} E_{y_{i}}+\sum_{k=1}^{m} \mathcal{E}_{\mathrm{T}_{k}} \sin \phi_{k} \\
\left(A_{1} / A_{\text {low }} \times \sum_{i=1}^{N_{\text {low }}} E_{\mathrm{T}_{i}}\right)-\mathcal{E}_{\mathrm{T}_{1}} \\
\vdots \\
\left(A_{m} / A_{\text {low }} \times \sum_{i=1}^{N_{\text {low }}} E_{\mathrm{T}_{i}}\right)-\mathcal{E}_{\mathrm{T}_{m}}
\end{array}\right)
$$

where the $A_{k}$ are the areas of each of the $m$ high- $E_{\mathrm{T}}$ patches, and $A_{\text {low }}$ is the summed area of the low- $E_{\mathrm{T}}$ patches. The first two lines impose the transverse momentum conservation constraints $\sum E_{x}=0$ and $\sum E_{y}=0$ on the pile-up contributions. On each of those two lines, the first sum (for which $N_{\text {low }}=N-m$ ) runs over the low- $E_{\mathrm{T}}$ patches, and the second over the estimated contributions of pile-up in the high- $E_{\mathrm{T}}$ patches. The quantities $\cos \phi_{k}$ and $\sin \phi_{k}$ are given by $E_{x_{k}} / E_{\mathrm{T}_{k}}$ and $E_{y_{k}} / E_{\mathrm{T}_{k}}$, respectively. Each of the remaining $m$ lines corresponds to one of the high- $E_{\mathrm{T}}$ patches, and penalizes any difference between the to-be-fitted pile-up estimate in that patch $\mathcal{E}_{\mathrm{T}_{k}}$ and the amount of pile-up transverse energy that would be expected in it, based on the event-wide average transverse energy density (as calculated from the low- $E_{\mathrm{T}}$ patches). The event-wide transverse energy is given by the term $A_{k} / A_{\text {low }} \times \sum_{i=1}^{N_{\text {low }}} E_{\mathrm{T}_{i}}$, where the ratio $\left(\sum_{i=1}^{N_{\text {low }}} E_{\mathrm{T}_{i}}\right) / A_{\text {low }}$ is the average energy density from the low- $E_{\mathrm{T}}$ patches.

For the determination of the covariance matrix, the fluctuations of all of the low- $E_{\mathrm{T}}$ patches are calculated from the contribution to the calorimeter energies due to detector resolution. The uncertainty in each measured patch transverse energy $E_{\mathrm{T}_{i}}$ is taken as

$$
\sigma_{i}^{2}=r_{0}^{2}+r^{2} E_{\mathrm{T}_{i}}
$$

where $r$ is the resolution scale, set to $0.5 \mathrm{GeV}^{1 / 2}$ and determined from calorimeter energy resolutions. The $r_{0}$ term is a resolution floor $(0.05 \mathrm{GeV})$, introduced to avoid numerical problems for events where most of the patches have no deposited $E_{\mathrm{T}}$. The patch sample variance $V_{\text {patch }}$ calculated above is used for the lower $m$ diagonal elements. The full covariance matrix is

$$
V=\left(\begin{array}{cccccc}
V_{11} & V_{12} & 0 & 0 & \ldots & 0 \\
V_{12} & V_{22} & 0 & 0 & \ldots & 0 \\
0 & 0 & s V_{\text {patch }} & 0 & \ldots & 0 \\
0 & 0 & 0 & \ddots & \ldots & 0 \\
\vdots & \vdots & \vdots & \vdots & \ddots & \vdots \\
0 & 0 & 0 & 0 & \ldots & s V_{\text {patch }}
\end{array}\right)
$$


where the upper $2 \times 2$ block is given by

$$
V_{\mathrm{cov}} \equiv\left(\begin{array}{ll}
V_{11} & V_{12} \\
V_{12} & V_{22}
\end{array}\right)=\left(\begin{array}{lll}
\sum_{i}^{N_{\text {low }}} \sigma_{i}^{2} \cos ^{2} \phi_{i} & \sum_{i}^{N_{\text {low }}} \sigma_{i}^{2} \cos \phi_{i} \sin \phi_{i} \\
\sum_{i}^{N_{\text {low }}} \sigma_{i}^{2} \cos \phi_{i} \sin \phi_{i} & \sum_{i}^{N_{\text {low }}} \sigma_{i}^{2} \sin ^{2} \phi_{i}
\end{array}\right),
$$

and $s=1$ is determined by optimizing the fit.

The minimization of the $\chi^{2}$ function with respect to the $\mathcal{E}_{\mathrm{T}_{k}}$ is performed analytically, avoiding computationally expensive numerical minimization. The solution involves solving an $m \times m$ linear system, where $m$, the number of above-threshold patches, is typically 4 or 5 .

Minimizing the function gives

$$
\begin{aligned}
0=\frac{1}{2} \frac{\partial \chi^{2}}{\partial \mathcal{E}_{\mathrm{T} k}}= & \left(\cos \phi_{k}, \sin \phi_{k}\right) V_{\mathrm{cov}}^{-1}\left(\begin{array}{l}
\sum_{i=1}^{N_{\text {low }}} E_{x_{i}}+\sum_{j=1}^{m} \mathcal{E}_{\mathrm{T}_{j}} \cos \phi_{j} \\
\sum_{i=1}^{N_{\text {low }}} E_{y_{i}}+\sum_{j=1}^{m} \mathcal{E}_{\mathrm{T}_{j}} \sin \phi_{j}
\end{array}\right) \\
& -\frac{1}{s V_{\text {patch }}}\left(\left(\frac{A_{k}}{A_{\text {low }}} \sum_{i=1}^{N_{\text {low }}} E_{\mathrm{T} i}\right)-\mathcal{E}_{\mathrm{T} k}\right) .
\end{aligned}
$$

The solution to this equation is given by the matrix equation

$$
\mathcal{E}_{\mathrm{T}_{k}}=\left[X^{-1}\right]_{k i} c_{i},
$$

where the $m \times m$ matrix

$$
X_{i k}=\left(\cos \phi_{i}, \sin \phi_{i}\right) V_{\mathrm{cov}}^{-1}\left(\begin{array}{c}
\cos \phi_{k} \\
\sin \phi_{k}
\end{array}\right)+\frac{\delta_{i k}}{s V_{\mathrm{patch}}}
$$

and

$$
c_{i}=\frac{A_{i}}{A_{\text {low }}} \frac{\sum_{j=1}^{N_{\text {low }}} E_{\mathrm{T}_{j}}}{s V_{\text {patch }}}-\left(\cos \phi_{i}, \sin \phi_{i}\right) V_{\text {cov }}^{-1}\left(\begin{array}{c}
\sum_{j=1}^{N_{\text {low }}} E_{x_{j}} \\
\sum_{j=1}^{N_{\text {low }}} E_{y_{j}}
\end{array}\right) .
$$

The $E_{\mathrm{T}}^{\text {miss }}$ for the event is then calculated from the $m$ high- $E_{\mathrm{T}}$ patches and the fit results for the pile-up contribution to the energy in these patches, where now

$$
\begin{aligned}
& E_{x}^{\mathrm{miss}}=-\sum_{k=1}^{m}\left(E_{x_{k}}-\mathcal{E}_{\mathrm{T}_{k}} \cos \phi_{k}\right), \\
& E_{y}^{\mathrm{miss}}=-\sum_{k=1}^{m}\left(E_{y_{k}}-\mathcal{E}_{\mathrm{T}_{k}} \sin \phi_{k}\right),
\end{aligned}
$$

and finally $E_{\mathrm{T}}^{\mathrm{miss}}=\sqrt{\left(E_{x}^{\mathrm{miss}}\right)^{2}+\left(E_{y}^{\mathrm{miss}}\right)^{2}}$.

\section{B Details of the offline reconstruction algorithms}

In the offline analyses, tracks with $p_{\mathrm{T}}>400 \mathrm{MeV}$ are reconstructed to identify a common vertex [42] and an event is required to have at least one such vertex. If more than one vertex is found in the event, the primary vertex is defined as the one with the largest $\sum_{t} p_{\mathrm{T}, t}^{2}$, where the sum runs over all tracks associated with the vertex. 
Electrons are reconstructed by matching tracks that originate from the primary vertex with clusters of energy deposits in the calorimeter [43]. They are required to have $p_{\mathrm{T}}>$ $25 \mathrm{GeV}$ and $|\eta|<2.47$. To suppress electrons that originate from the hadronic showers of jets, the electron is also required to be isolated using information from the calorimeter and tracks within a cone of size $\Delta R=0.2$ around the electron direction. The total efficiency of electron reconstruction and isolation is $85 \%$ for an electron with $p_{\mathrm{T}} \approx 30 \mathrm{GeV}$.

Muons are reconstructed by matching tracks from the muon spectrometer with innerdetector tracks that are associated with the primary vertex [44]. Muons are required to have $p_{\mathrm{T}}>25 \mathrm{GeV}$ and $|\eta|<2.47$, and they are required to be isolated. For the isolation working point used, the efficiency for a muon with $p_{\mathrm{T}}$ in the range $20-100 \mathrm{GeV}$ is $96 \%$.

Jets are reconstructed starting with information from the calorimeter. Cells are grouped into topological clusters [13], described in section 3.3, which in turn are used to reconstruct jets using the anti- $k_{t}$ algorithm with a radius parameter of $R=0.4$. Jets are required to have $p_{\mathrm{T}}>20 \mathrm{GeV}$ and $|\eta|<4.5$. To suppress jets that come from pile-up, those that have $|\eta|<2.4$ are required to exceed the jet vertex tagger (JVT) threshold [25]. The JVT uses tracks and vertices to assign a likelihood for a jet to be associated with the primary vertex. Jets that fail the JVT requirement are removed, as they are likely to be due to pile-up. The efficiency to select a jet from a signal process is $92 \%$, while jets originating from pile-up collisions are rejected $99 \%$ of the time in the $p_{\mathrm{T}}$ range $20-50 \mathrm{GeV}$. Each jet with $|\eta|<2.5$ is evaluated for the likelihood that it originated from a $b$-hadron [45]. The efficiency for tagging a jet containing a $b$-hadron is $77 \%$ for jets originating from $t \bar{t}$ events [46].

When selecting events based on the number of jets and leptons (electrons or muons), it is necessary to resolve the ambiguity for cases where a reconstructed jet and a lepton both result from the same detector signals. To remove such ambiguities, a sequential overlap removal procedure is defined. If the jet is within a cone of size $\Delta R=0.2$ around any electron, the jet is removed. If a jet is found within a conical annulus defined by $0.2<\Delta R<0.4$, then the electron is removed. If there is a muon within $\Delta R=0.4$ of a jet, and the jet has at least three charged tracks with $p_{\mathrm{T}}>500 \mathrm{MeV}$, then the muon is removed; otherwise the jet is removed.

\section{The cell $E_{\mathrm{T}}^{\text {miss }}$ background distribution model}

The details of the background cell $E_{\mathrm{T}}^{\text {miss }}$ distribution model discussed in section 5.1 are as follows. The model has two components. The first covers the bulk of the distribution, which is assumed to originate from resolution effects. The second component, which dominates in the tail, comes from rare occurrences which scale linearly with the luminosity, and hence, for fixed beam parameters, linearly with the instantaneous average number $\mu$ of interactions per bunch crossing.

Component 1: $\boldsymbol{\Sigma} \boldsymbol{E}_{\mathrm{T}}$ resolution-dependent bulk distribution. The bulk resolution effects are assumed to depend on the total transverse energy deposited in the calorimeter, $\Sigma E_{\mathrm{T}}$. It is assumed that the two perpendicular components of $E_{\mathrm{T}}^{\text {miss }}$ are independently identically Gaussian distributed with a width $\sigma\left(\Sigma E_{\mathrm{T}}\right)$ that depends on $\Sigma E_{\mathrm{T}}$, such that 


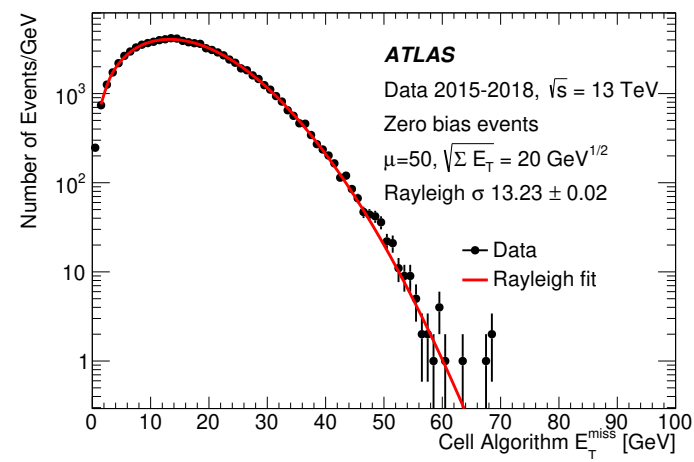

(a)

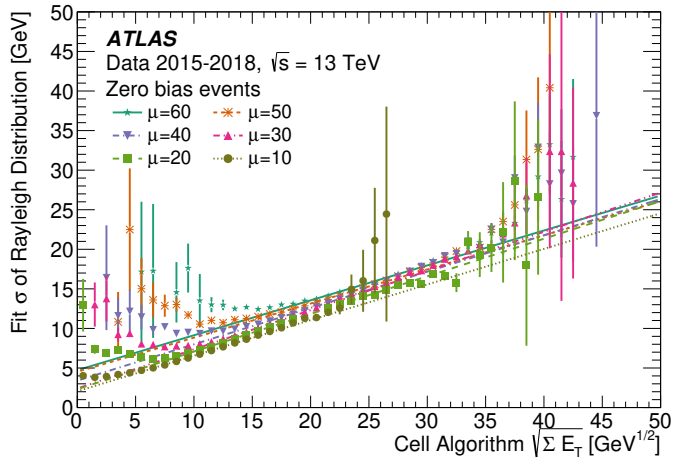

(b)

Figure 13. Data and fits to data for $E_{\mathrm{T}}^{\mathrm{miss}}$ model. (a) A comparison of the measured cell-algorithm $E_{\mathrm{T}}^{\text {miss }}$ distribution with a Rayleigh distribution fit, for a narrow range of pile-up centred on $\langle\mu\rangle \approx 50$. The black histogram is the distribution from the unbiased data. The red curve is the result of a fit to a Rayleigh distribution. (b) Values of $\sigma$ are shown from Rayleigh fits to $E_{\mathrm{T}}^{\text {miss }}$ distributions for 6 values of $\langle\mu\rangle$ and 50 values of $\sqrt{\Sigma E_{\mathrm{T}}}$ per $\langle\mu\rangle$ value, as well as the data from which the fits are obtained. Also shown are straight-line fits to the $50 \sqrt{\Sigma E_{\mathrm{T}}}$ bins for each $\langle\mu\rangle$.

events with a given $\Sigma E_{\mathrm{T}}$ will obey a Rayleigh distribution in $E_{\mathrm{T}}^{\text {miss }}$,

$$
R\left(E_{\mathrm{T}}^{\text {miss }} \mid \sigma\left(\Sigma E_{\mathrm{T}}\right)\right)=\left(E_{\mathrm{T}}^{\text {miss }} / \sigma^{2}\right) \exp \left(-\left[E_{\mathrm{T}}^{\text {miss }}\right]^{2} / 2 \sigma^{2}\right) .
$$

In turn the probability density function of $\Sigma E_{\mathrm{T}}$ is assumed to be a function $P\left(\Sigma E_{\mathrm{T}} \mid \mu, \vec{\gamma}\right)$, where $\mu$ is the instantaneous average number of interactions per bunch crossing and $\vec{\gamma}$ is used to parameterize any other dependencies. For a given $\mu, E_{\mathrm{T}}^{\text {miss }}$ is distributed according to the probability density function

$$
P_{\text {res }}\left(E_{\mathrm{T}}^{\text {miss }} \mid \mu\right)=\int_{0}^{\infty} R\left(E_{\mathrm{T}}^{\mathrm{miss}} \mid \sigma\left(\Sigma E_{\mathrm{T}}\right)\right) P\left(\Sigma E_{\mathrm{T}} \mid \mu, \vec{\gamma}\right) \mathrm{d}\left(\Sigma E_{\mathrm{T}}\right) .
$$

In order to sample from the distribution $P_{\text {res }}\left(E_{\mathrm{T}}^{\text {miss }} \mid \mu\right)$, expressions for both $\sigma\left(\Sigma E_{\mathrm{T}}\right)$ and $P\left(\Sigma E_{\mathrm{T}} \mid \mu, \vec{\gamma}\right)$ are needed.

Fits to find $\sigma\left(\Sigma E_{\mathrm{T}}\right)$ are discussed first. Figure 13a shows an example cell $E_{\mathrm{T}}^{\text {miss }}$ distribution for a narrow range around $\langle\mu\rangle \approx 50$ and $\Sigma E_{\mathrm{T}} \approx 400 \mathrm{GeV}$. The plot also shows a Rayleigh fit to the $E_{\mathrm{T}}^{\text {miss }}$ distribution; the fit characterizes it well except at the highest $E_{\mathrm{T}}^{\mathrm{miss}}$ values. The points in figure $13 \mathrm{~b}$ show the values of $\sigma$ obtained from fits to the $E_{\mathrm{T}}^{\text {miss }}$ distribution for 6 values of $\langle\mu\rangle$ and 50 bins of $\left(\Sigma E_{\mathrm{T}}\right)^{1 / 2}$ for each $\langle\mu\rangle$. Although some $\langle\mu\rangle$ dependence is visible, the model provides a reasonable description of the data using a parameterization of the resolution given by $\sigma\left(\Sigma E_{\mathrm{T}}\right)=\alpha_{1}+\alpha_{2} \sqrt{\Sigma E_{\mathrm{T}}}$ with $\alpha_{1}=3 \mathrm{GeV}$ and $\alpha_{2}=0.465 \mathrm{GeV}^{1 / 2}$. Plots like those in figure $13 \mathrm{~b}$ show that this linear fit is a good model for the bulk of events and performs poorly only in ranges of the total transverse energy where the Rayleigh fits do not provide a good description of the $E_{\mathrm{T}}^{\text {miss }}$.

To find the $\Sigma E_{\mathrm{T}}$ probability density function $P\left(\Sigma E_{\mathrm{T}} \mid \mu, \vec{\gamma}\right)$, one may first consider the distribution $S_{1}\left(\Sigma E_{\mathrm{T}} \mid \vec{\gamma}\right)$ of the $\Sigma E_{\mathrm{T}}$ for a single proton-proton interaction. The corresponding distribution $S_{n}\left(\Sigma E_{\mathrm{T}} \mid \vec{\gamma}\right)$ for $n$ simultaneous proton-proton interactions is given by the 
$n$-fold convolution of $S_{1}\left(\Sigma E_{\mathrm{T}} \mid \vec{\gamma}\right)$. The values of $n$ that are sampled for any $\mu$ are assumed to be Poisson distributed with mean $\mu$, so that the resulting distribution $P\left(\Sigma E_{\mathrm{T}} \mid \mu, \vec{\gamma}\right)$ is given by a Poisson-weighted sum of terms, each term being the product of the Poisson probability for $n$ interactions given $\mu$ with the corresponding $S_{n}\left(\Sigma E_{\mathrm{T}} \mid \vec{\gamma}\right)$ :

$$
P\left(\Sigma E_{\mathrm{T}} \mid \mu, \vec{\gamma}\right)=\sum_{n=1}^{\infty} \operatorname{Poi}(n \mid \mu) S_{n}\left(\Sigma E_{\mathrm{T}} \mid \vec{\gamma}\right) .
$$

In the absence of data taken at low $\langle\mu\rangle$ under the same beam conditions, $S_{1}\left(\Sigma E_{\mathrm{T}} \mid \vec{\gamma}\right)$ cannot be determined directly. Instead, various models of $S_{1}\left(\Sigma E_{\mathrm{T}} \mid \vec{\gamma}\right)$ are explored by varying their parameters until the calculation gives a reasonable match to the measured high-luminosity $P\left(\Sigma E_{\mathrm{T}} \mid \mu, \vec{\gamma}\right)$ distributions. It is found that a single-interaction $\Sigma E_{\mathrm{T}}$ distribution of the form

$$
S_{1}\left(\Sigma E_{\mathrm{T}} \mid \vec{\gamma}\right)=\gamma_{3} \gamma_{1} \exp \left(-\gamma_{1} \Sigma^{\prime}\right)+\left(1-\gamma_{3}\right) \gamma_{2} \exp \left(-\gamma_{2} \Sigma^{\prime}\right)
$$

gives a result that matches the data well for $\mu$ of about 20 or greater. The parameters of this expression are $\gamma_{1}=0.070 \mathrm{GeV}^{-1}, \gamma_{2}=0.009 \mathrm{GeV}^{-1}, \gamma_{3}=0.995$, and for the $\mu$ dependent substitution $\Sigma^{\prime}=\Sigma E_{\mathrm{T}}-\left(\gamma_{4}+\gamma_{5} \times \mu\right)$ with $\gamma_{4}=25 \mathrm{GeV}$ and $\gamma_{5}=-5 \mathrm{GeV}$. Figure 14 shows an example of the $\Sigma E_{\mathrm{T}}$ modelling for $\langle\mu\rangle \approx 50$.

The $n$-fold convolution can be carried out analytically only for a limited number of functions. For example, the single-interaction $\Sigma E_{\mathrm{T}}$ distribution for the LHC Run 1 was approximated by a single exponential function, and the convolution was then performed as described in ref. [47]. For the more complicated functions used in the present calculation, the convolution and Poisson sum is computed using a fast Fourier transform and the method described in refs. [48] and [49].

The Fourier transform $\widetilde{P}(\omega \mid \mu, \vec{\gamma})$ of the weighted convolution sum $P\left(\Sigma E_{\mathrm{T}} \mid \mu, \vec{\gamma}\right)$ is rewritten as the infinite sum of Fourier transforms over $S_{1}\left(\Sigma E_{\mathrm{T}} \mid \vec{\gamma}\right)$, denoted by $\widetilde{S_{1}}(\omega \mid \vec{\gamma})$. This facilitates calculation, as the infinite sum can be performed using the Fourier transform:

$$
\begin{aligned}
\widetilde{P}(\omega \mid \mu, \vec{\gamma}) & =\sum_{n=1}^{\infty} \operatorname{Poi}(n \mid \mu) \widetilde{S_{n}}(\omega \mid \vec{\gamma}) \\
& =\sum_{n=1}^{\infty} \operatorname{Poi}(n \mid \mu)(2 \pi)^{n-1}\left(\widetilde{S_{1}}(\omega \mid \vec{\gamma})\right)^{n} \\
& =\frac{1}{2 \pi} \mathrm{e}^{-\mu} \sum_{n=1}^{\infty}(2 \pi)^{n} \mu^{n}\left(\widetilde{S_{1}}(\omega \mid \vec{\gamma})\right)^{n} / n ! \\
& =\frac{1}{2 \pi} \mathrm{e}^{-\mu}\left(\sum_{n=0}^{\infty}\left[2 \pi \mu \widetilde{S_{1}}(\omega \mid \vec{\gamma})\right]^{n} / n !-1\right) \\
& =\frac{1}{2 \pi} \mathrm{e}^{-\mu}\left(\exp \left(2 \pi \mu \widetilde{S_{1}}(\omega \mid \vec{\gamma})\right)-1\right) .
\end{aligned}
$$

In principle, one could also take a logarithm of the exponential term in the final expression above to directly solve for $\widetilde{S_{1}}(\omega \mid \vec{\gamma})$ from $\widetilde{P}(\omega \mid \mu, \vec{\gamma})$. However, the uncertainties in the $P\left(\Sigma E_{\mathrm{T}} \mid \mu, \vec{\gamma}\right)$ distribution are amplified in this process, making it impossible in practice to obtain useful results in this way. 


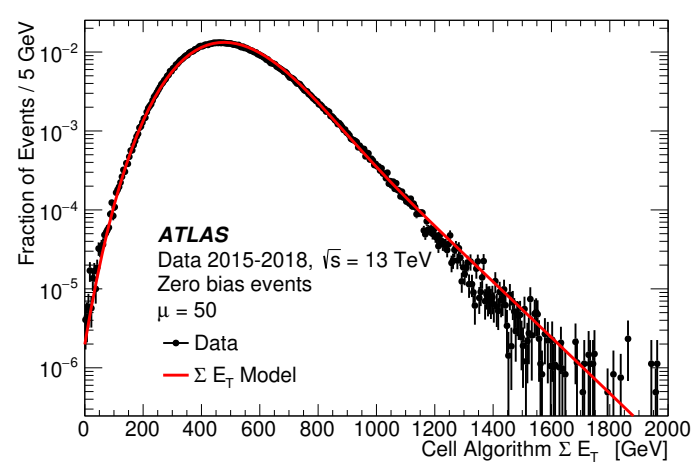

Figure 14. The probability density function $P\left(\Sigma E_{\mathrm{T}} \mid \mu, \vec{\gamma}\right)$ described in the text is shown and compared with data recorded using a zero bias trigger. The model and the data both correspond to pile-up of $\langle\mu\rangle \approx 50$.

Component 2: luminosity-dependent tail from rare events. The Rayleigh distribution resolution model described above is found to describe the observed cell $E_{\mathrm{T}}^{\text {miss }}$ distribution well for $E_{\mathrm{T}}^{\text {miss }}$ up to about $50 \mathrm{GeV}$, but not beyond. A second component, which contributes more at larger $E_{\mathrm{T}}^{\text {miss }}$ values, is assumed to come from a combination of events with rare calorimeter measurement fluctuations, for example very poor measurement of a single jet in a two-jet event, and from events containing non-interacting particles, such as those in which semileptonic $b$-hadron decays produce neutrinos. The probability of such an event is expected to scale linearly with luminosity, and hence for fixed bunch-crossing rate, to scale linearly with $\mu$. Indeed, a Fréchet function that is linearly dependent on $\mu$,

$$
\mu F\left(E_{\mathrm{T}}^{\mathrm{miss}} \mid m, \alpha, s\right)=\mu A \frac{\alpha}{s}\left(\frac{E_{\mathrm{T}}^{\mathrm{miss}}-m}{s}\right)^{-1-\alpha} \exp \left[-\left(\frac{E_{\mathrm{T}}^{\mathrm{miss}}-m}{s}\right)^{-\alpha}\right]
$$

is found to describe the high $E_{\mathrm{T}}^{\text {miss }}$ distribution, with parameters $\alpha=3.54, s=8 \mathrm{GeV}$, and $m=40 \mathrm{GeV}$, and with the probability per single $p p$ interaction of such a tail event being $A=10^{-5}$.

Full $\boldsymbol{E}_{\mathbf{T}}^{\text {miss }}$ distribution. The complete model $E_{\mathrm{T}}^{\text {miss }}$ distribution is then obtained by vector addition of the two $E_{\mathrm{T}}^{\text {miss }}$ sources. A random azimuthal angle $\phi_{\text {rand }}$ is assumed between the two vectors and the magnitude of the $E_{\mathrm{T}}^{\mathrm{miss}}$ is sampled from two sources. These are (i) the $E_{\mathrm{T}}^{\text {miss }}$ distribution in eq. (C.1) from the resolution function of the total transverse energy in eq. (C.2), and (ii) a tail modelled with the Fréchet distribution, depending linearly upon $\mu$, in eq. (C.3). The combination yields

$$
\text { Event } E_{\mathrm{T}}^{\text {miss }}=\mid E_{\mathrm{T}}^{\text {miss }}(\text { resolution }) \hat{\mathbf{n}}_{\mathrm{T}}(\phi=0)+E_{\mathrm{T}}^{\text {miss }}(\text { tail }) \hat{\mathbf{n}}_{\mathrm{T}}\left(\phi=\phi_{\text {rand }}\right) \mid .
$$

It is the distribution of this quantity that is compared with data in section 5.1.

Open Access. This article is distributed under the terms of the Creative Commons Attribution License (CC-BY 4.0), which permits any use, distribution and reproduction in any medium, provided the original author(s) and source are credited. 


\section{References}

[1] ATLAS collaboration, Performance of the ATLAS trigger system in 2015, Eur. Phys. J. C 77 (2017) 317 [arXiv: 1611.09661] [INSPIRE].

[2] ATLAS collaboration, The ATLAS experiment at the CERN Large Hadron Collider, 2008 JINST 3 S08003 [INSPIRE].

[3] ATLAS collaboration, Combination of searches for invisible Higgs boson decays with the ATLAS experiment, Phys. Rev. Lett. 122 (2019) 231801 [arXiv:1904.05105] [INSPIRE].

[4] ATLAS collaboration, Search for invisible Higgs boson decays in vector boson fusion at $\sqrt{s}=13 \mathrm{TeV}$ with the ATLAS detector, Phys. Lett. B 793 (2019) 499 [arXiv:1809.06682] [INSPIRE].

[5] ATLAS collaboration, Search for charged Higgs bosons decaying via $H^{ \pm} \rightarrow \tau^{ \pm} \nu_{\tau}$ in the $\tau+$ jets and $\tau+$ lepton final states with $36 \mathrm{fb}^{-1}$ of pp collision data recorded at $\sqrt{\mathrm{s}}=13 \mathrm{TeV}$ with the ATLAS experiment, JHEP 09 (2018) 139 [arXiv:1807.07915] [INSPIRE].

[6] ATLAS collaboration, Search for dark matter and other new phenomena in events with an energetic jet and large missing transverse momentum using the ATLAS detector, JHEP 01 (2018) 126 [arXiv: 1711.03301] [INSPIRE].

[7] ATLAS collaboration, Search for squarks and gluinos in final states with hadronically decaying $\tau$-leptons, jets, and missing transverse momentum using pp collisions at $\sqrt{s}=13 \mathrm{TeV}$ with the ATLAS detector, Phys. Rev. D 99 (2019) 012009 [arXiv:1808.06358] [INSPIRE].

[8] ATLAS collaboration, Searches for electroweak production of supersymmetric particles with compressed mass spectra in $\sqrt{s}=13$ TeV pp collisions with the ATLAS detector, Phys. Rev. D 101 (2020) 052005 [arXiv:1911.12606] [INSPIRE].

[9] ATLAS collaboration, Search for new phenomena using the invariant mass distribution of same-flavour opposite-sign dilepton pairs in events with missing transverse momentum in $\sqrt{s}=13 \mathrm{TeV}$ pp collisions with the ATLAS detector, Eur. Phys. J. C 78 (2018) 625 [arXiv: 1805.11381] [INSPIRE].

[10] ATLAS collaboration, Search for heavy charged long-lived particles in the ATLAS detector in $36.1 \mathrm{fb}^{-1}$ of proton-proton collision data at $\sqrt{s}=13 \mathrm{TeV}$, Phys. Rev. D 99 (2019) 092007 [arXiv: 1902.01636] [INSPIRE].

[11] ATLAS collaboration, Observation of $H \rightarrow b \bar{b}$ decays and $V H$ production with the ATLAS detector, Phys. Lett. B $\mathbf{7 8 6}$ (2018) 59 [arXiv:1808.08238] [INSPIRE].

[12] ATLAS collaboration, Readiness of the ATLAS tile calorimeter for LHC collisions, Eur. Phys. J. C 70 (2010) 1193 [arXiv:1007.5423] [InSPIRE].

[13] ATLAS collaboration, Topological cell clustering in the ATLAS calorimeters and its performance in LHC Run 1, Eur. Phys. J. C 77 (2017) 490 [arXiv: 1603. 02934] [InSPIRE].

[14] ATLAS IBL collaboration, Production and integration of the ATLAS Insertable B-Layer, 2018 JINST 13 T05008 [arXiv: 1803.00844] [INSPIRE].

[15] R. Achenbach et al., The ATLAS level-1 calorimeter trigger, 2008 JINST 3 P03001 [INSPIRE]. 
[16] ATLAS collaboration, Performance of missing transverse momentum reconstruction with the ATLAS detector using proton-proton collisions at $\sqrt{s}=13$ TeV, Eur. Phys. J. C 78 (2018) 903 [arXiv: 1802.08168] [INSPIRE].

[17] ATLAS collaboration, $E_{T}^{\text {miss }}$ performance in the ATLAS detector using 2015-2016 LHC pp collisions, ATLAS-CONF-2018-023, CERN, Geneva, Switzerland (2018).

[18] ATLAS collaboration, Trigger menu in 2018, ATL-DAQ-PUB-2019-001, CERN, Geneva, Switzerland (2019).

[19] ATLAS collaboration, Performance of the upgraded PreProceSSOR of the ATLAS level-1 calorimeter trigger, CERN-EP-2020-042, CERN, Geneva, Switzerland (2020) [arXiv: 2005. 04179] [INSPIRE].

[20] ATLAS collaboration, Readiness of the ATLAS liquid argon calorimeter for LHC collisions, Eur. Phys. J. C 70 (2010) 723 [arXiv:0912.2642] [INSPIRE].

[21] ATLAS collaboration, Performance of algorithms that reconstruct missing transverse momentum in $\sqrt{s}=8 \mathrm{TeV}$ proton-proton collisions in the ATLAS detector, Eur. Phys. J. C 77 (2017) 241 [arXiv: 1609.09324] [INSPIRE].

[22] M. Cacciari, G.P. Salam and G. Soyez, The anti-k $k_{t}$ jet clustering algorithm, JHEP 04 (2008) 063 [arXiv:0802.1189] [INSPIRE].

[23] M. Cacciari, G.P. Salam and G. Soyez, FastJet user manual, Eur. Phys. J. C 72 (2012) 1896 [arXiv: 1111.6097] [INSPIRE].

[24] M. Cacciari, G.P. Salam and G. Soyez, The catchment area of jets, JHEP 04 (2008) 005 [arXiv:0802.1188] [INSPIRE].

[25] ATLAS collaboration, Performance of pile-up mitigation techniques for jets in pp collisions at $\sqrt{s}=8$ TeV using the ATLAS detector, Eur. Phys. J. C 76 (2016) 581 [arXiv: 1510.03823] [INSPIRE].

[26] ATLAS collaboration, Jet energy scale measurements and their systematic uncertainties in proton-proton collisions at $\sqrt{s}=13 \mathrm{TeV}$ with the ATLAS detector, Phys. Rev. D 96 (2017) 072002 [arXiv: 1703.09665] [INSPIRE].

[27] M. Cacciari and G.P. Salam, Pileup subtraction using jet areas, Phys. Lett. B 659 (2008) 119 [arXiv: 0707.1378] [INSPIRE].

[28] ATLAS collaboration, Identification and rejection of pile-up jets at high pseudorapidity with the ATLAS detector, Eur. Phys. J. C 77 (2017) 580 [Erratum ibid. 77 (2017) 712] [arXiv: 1705. 02211] [INSPIRE].

[29] ATLAS collaboration, Technical design report for the phase-II upgrade of the ATLAS TDAQ system, CERN-LHCC-2017-020, CERN, Geneva, Switzerland (2017) [ATLAS-TDR-029].

[30] T. Gleisberg et al., Event generation with SHERPA 1.1, JHEP 02 (2009) 007 [arXiv:0811.4622] [INSPIRE].

[31] T. Gleisberg and S. Höche, Comix, a new matrix element generator, JHEP 12 (2008) 039 [arXiv:0808.3674] [INSPIRE].

[32] F. Cascioli, P. Maierhöfer and S. Pozzorini, Scattering amplitudes with open loops, Phys. Rev. Lett. 108 (2012) 111601 [arXiv:1111.5206] [INSPIRE].

[33] S. Schumann and F. Krauss, A parton shower algorithm based on Catani-Seymour dipole factorisation, JHEP 03 (2008) 038 [arXiv:0709.1027] [INSPIRE]. 
[34] S. Höche, F. Krauss, M. Schönherr and F. Siegert, QCD matrix elements + parton showers: the NLO case, JHEP 04 (2013) 027 [arXiv: 1207.5030] [INSPIRE].

[35] NNPDF collaboration, Parton distributions for the LHC Run II, JHEP 04 (2015) 040 [arXiv: 1410.8849] [INSPIRE].

[36] ATLAS collaboration, ATLAS simulation of boson plus jets processes in Run II, ATL-PHYS-PUB-2017-006, CERN, Geneva, Switzerland (2017).

[37] ATLAS collaboration, The ATLAS simulation infrastructure, Eur. Phys. J. C 70 (2010) 823 [arXiv:1005.4568] [INSPIRE].

[38] GEANT4 collaboration, GEANT4 - a simulation toolkit, Nucl. Instrum. Meth. A 506 (2003) 250 [INSPIRE].

[39] T. Sjöstrand, S. Mrenna and P.Z. Skands, A brief introduction to PYTHIA 8.1, Comput. Phys. Commun. 178 (2008) 852 [arXiv:0710.3820] [INSPIRE].

[40] ATLAS collaboration, The PYTHIA 8 A3 tune description of ATLAS minimum bias and inelastic measurements incorporating the Donnachie-Landshoff diffractive model, ATL-PHYS-PUB-2016-017, CERN, Geneva, Switzerland (2016).

[41] P. Skands, S. Carrazza and J. Rojo, Tuning PYTHIA 8.1: the Monash 2013 tune, Eur. Phys. J. $C 74$ (2014) 3024 [arXiv:1404.5630] [INSPIRE].

[42] ATLAS collaboration, Reconstruction of primary vertices at the ATLAS experiment in Run 1 proton-proton collisions at the LHC, Eur. Phys. J. C 77 (2017) 332 [arXiv:1611.10235] [INSPIRE].

[43] ATLAS collaboration, Electron and photon performance measurements with the ATLAS detector using the 2015-2017 LHC proton-proton collision data, 2019 JINST 14 P12006 [arXiv: 1908.00005] [INSPIRE].

[44] ATLAS collaboration, Muon reconstruction performance of the ATLAS detector in proton-proton collision data at $\sqrt{s}=13$ TeV, Eur. Phys. J. C 76 (2016) 292 [arXiv: 1603.05598] [INSPIRE].

[45] ATLAS collaboration, Measurements of b-jet tagging efficiency with the ATLAS detector using $t \bar{t}$ events at $\sqrt{s}=13$ TeV, JHEP 08 (2018) 089 [arXiv:1805.01845] [INSPIRE].

[46] ATLAS collaboration, ATLAS b-jet identification performance and efficiency measurement with $t \bar{t}$ events in pp collisions at $\sqrt{s}=13$ TeV, Eur. Phys. J. C 79 (2019) 970 [arXiv: 1907.05120] [INSPIRE].

[47] ATLAS collaboration, Analytical description of missing transverse-momentum trigger rates in ATLAS with 7 and 8 TeV data, ATL-DAQ-PUB-2017-002, CERN, Geneva, Switzerland (2017).

[48] H. Hu and J. Nielsen, Analytic confidence level calculations using the likelihood ratio and Fourier transform, physics/9906010 [INSPIRE].

[49] K.S. Cranmer, B. Mellado, W. Quayle and S.L. Wu, Challenges in moving the LEP Higgs statistics to the LHC, eConf C 030908 (2003) MODT004 [physics/0312050] [INSPIRE].

[50] ATLAS collaboration, ATLAS computing acknowledgements, ATL-SOFT-PUB-2020-001, CERN, Geneva, Switzerland (2020). 


\section{The ATLAS collaboration}

G. Aad ${ }^{102}$, B. Abbott ${ }^{128}$, D.C. Abbott ${ }^{103}$, A. Abed Abud ${ }^{36}$, K. Abeling ${ }^{53}$, D.K. Abhayasinghe ${ }^{94}$, S.H. Abidi ${ }^{166}$, O.S. AbouZeid ${ }^{40}$, N.L. Abraham ${ }^{155}$, H. Abramowicz 160, H. Abreu 159, Y. Abulaiti ${ }^{6}$, B.S. Acharya 67a,67b,o, B. Achkar ${ }^{53}$, L. Adam ${ }^{100}$, C. Adam Bourdarios 5, L. Adamczyk ${ }^{84 a}$, L. Adamek 166, J. Adelman 121, M. Adersberger ${ }^{114}$, A. Adiguzel ${ }^{12 c}$, S. Adorni ${ }^{54}$, T. Adye ${ }^{143}$, A.A. Affolder ${ }^{145}$, Y. Afik 159, C. Agapopoulou ${ }^{65}$, M.N. Agaras 38, A. Aggarwal 119, C. Agheorghiesei 27c, J.A. Aguilar-Saavedra 139f,139a,af, A. Ahmad ${ }^{36}$, F. Ahmadov ${ }^{80}$, W.S. Ahmed ${ }^{104}$, X. Ai ${ }^{18}$, G. Aielli ${ }^{74 a, 74 b}$, S. Akatsuka ${ }^{86}$, T.P.A. Åkesson ${ }^{97}$, E. Akilli ${ }^{54}$, A.V. Akimov ${ }^{111}$, K. Al Khoury ${ }^{65}$, G.L. Alberghi 23b,23a, J. Albert ${ }^{175}$, M.J. Alconada Verzini ${ }^{160}$, S. Alderweireldt ${ }^{36}$, M. Aleksa ${ }^{36}$, I.N. Aleksandrov ${ }^{80}$, C. Alexa ${ }^{27 b}$, T. Alexopoulos ${ }^{10}$, A. Alfonsi ${ }^{120}$, F. Alfonsi 23b,23a, M. Alhroob ${ }^{128}$, B. Ali 141, S. Ali ${ }^{157}$, M. Aliev ${ }^{165}$, G. Alimonti ${ }^{69 a}$, C. Allaire ${ }^{36}$, B.M.M. Allbrooke ${ }^{155}$, B.W. Allen 131, P.P. Allport ${ }^{21}$, A. Aloisio ${ }^{70 a, 70 b}$, F. Alonso ${ }^{89}$, C. Alpigiani ${ }^{147}$, A.A. Alshehri ${ }^{57}$,

E. Alunno Camelia 74a,74b, M. Alvarez Estevez ${ }^{99}$, M.G. Alviggi 70a,70b, Y. Amaral Coutinho 81b, A. Ambler 104, L. Ambroz 134, C. Amelung 26, D. Amidei 106, S.P. Amor Dos Santos 139a, S. Amoroso 46, C.S. Amrouche ${ }^{54}$, F. An ${ }^{79}$, C. Anastopoulos ${ }^{148}$, N. Andari ${ }^{144}$, T. Andeen ${ }^{11}$, C.F. Anders 61b, J.K. Anders ${ }^{20}$, S.Y. Andrean 45a,45b, A. Andreazza 69a,69b, V. Andrei 61a, C.R. Anelli 175, S. Angelidakis 9, A. Angerami 39, A.V. Anisenkov 122b,122a, A. Annovi ${ }^{72 a}$, C. Antel ${ }^{54}$, M.T. Anthony ${ }^{148}$, E. Antipov ${ }^{129}$, M. Antonelli 51, D.J.A. Antrim 170, F. Anulli ${ }^{73 a}$, M. Aoki ${ }^{82}$, J.A. Aparisi Pozo ${ }^{173}$, M.A. Aparo ${ }^{155}$, L. Aperio Bella ${ }^{15 a}$, V. Araujo Ferraz ${ }^{81 b}$, R. Araujo Pereira ${ }^{81 b}$, C. Arcangeletti 51, A.T.H. Arce ${ }^{49}$, F.A. Arduh 89, J-F. Arguin ${ }^{110}$, S. Argyropoulos 52, J.-H. Arling 46, A.J. Armbruster ${ }^{36}$, A. Armstrong ${ }^{170}$, O. Arnaez ${ }^{166}$, H. Arnold ${ }^{120}$, Z.P. Arrubarrena Tame ${ }^{114}$, G. Artoni ${ }^{134}$, S. Artz ${ }^{100}$, S. Asai 162, T. Asawatavonvanich 164, N. Asbah 59, E.M. Asimakopoulou 171, L. Asquith ${ }^{155}$, J. Assahsah 35d, K. Assamagan 29, R. Astalos 28a, R.J. Atkin 33a, M. Atkinson ${ }^{172}$, N.B. Atlay ${ }^{19}$, H. Atmani 65, K. Augsten 141, G. Avolio 36, M.K. Ayoub 15a, G. Azuelos 110,an, H. Bachacou 144, K. Bachas 161, M. Backes 134, F. Backman 45a,45b, P. Bagnaia ${ }^{73 a, 73 b}$, M. Bahmani ${ }^{85}$, H. Bahrasemani ${ }^{151}$, A.J. Bailey ${ }^{173}$, V.R. Bailey ${ }^{172}$, J.T. Baines ${ }^{143}$ C. Bakalis ${ }^{10}$, O.K. Baker ${ }^{182}$, P.J. Bakker ${ }^{120}$, E. Bakos ${ }^{16}$, D. Bakshi Gupta ${ }^{8}$, S. Balaji ${ }^{156}$, E.M. Baldin 122b,122a, P. Balek ${ }^{179}$, F. Balli ${ }^{144}$, W.K. Balunas ${ }^{134}$, J. Balz ${ }^{100}$, E. Banas ${ }^{85}$, M. Bandieramonte ${ }^{138}$, A. Bandyopadhyay ${ }^{24}$, Sw. Banerjee ${ }^{180, j}$, L. Barak ${ }^{160}$, W.M. Barbe ${ }^{38}$, E.L. Barberio ${ }^{105}$, D. Barberis ${ }^{55 \mathrm{~b}, 55 \mathrm{a}}$, M. Barbero ${ }^{102}$, G. Barbour ${ }^{95}$, T. Barillari ${ }^{115}$, M-S. Barisits ${ }^{36}$, J. Barkeloo 131, T. Barklow ${ }^{152}$, R. Barnea ${ }^{159}$, B.M. Barnett ${ }^{143}$, R.M. Barnett ${ }^{18}$, Z. Barnovska-Blenessy ${ }^{60 a}$, A. Baroncelli 60a, G. Barone ${ }^{29}$, A.J. Barr ${ }^{134}$, L. Barranco Navarro 45a,45b, F. Barreiro ${ }^{99}$, J. Barreiro Guimarães da Costa 15a, U. Barron ${ }^{160}$, S. Barsov 137, F. Bartels ${ }^{61 a}$, R. Bartoldus ${ }^{152}$, G. Bartolini 102, A.E. Barton 90, P. Bartos ${ }^{28 a}$, A. Basalaev ${ }^{46}$, A. Basan ${ }^{100}$, A. Bassalat ${ }^{65, a k}$, M.J. Basso ${ }^{166}$, R.L. Bates ${ }^{57}$, S. Batlamous 35e, J.R. Batley ${ }^{32}$, B. Batool ${ }^{150}$, M. Battaglia ${ }^{145}$, M. Bauce ${ }^{73 a, 73 b}$, F. Bauer ${ }^{144}$, K.T. Bauer ${ }^{170}$, H.S. Bawa ${ }^{31}$, J.B. Beacham ${ }^{49}$, T. Beau ${ }^{135}$, P.H. Beauchemin ${ }^{169}$, F. Becherer ${ }^{52}$, P. Bechtle ${ }^{24}$, H.C. Beck ${ }^{53}$, H.P. Beck ${ }^{20, r}$, K. Becker ${ }^{177}$, C. Becot ${ }^{46}$, A. Beddall ${ }^{12 d}$, A.J. Beddall ${ }^{12 a}$, V.A. Bednyakov ${ }^{80}$, M. Bedognetti ${ }^{120}$, C.P. Bee ${ }^{154}$, T.A. Beermann ${ }^{181}$, M. Begalli ${ }^{81 b}$, M. Begel ${ }^{29}$, A. Behera ${ }^{154}$, J.K. Behr ${ }^{46}$, F. Beisiegel ${ }^{24}$, M. Belfkir ${ }^{5}$, A.S. Bell ${ }^{95}$, G. Bella ${ }^{160}$, L. Bellagamba ${ }^{23 b}$, A. Bellerive ${ }^{34}$, P. Bellos ${ }^{9}$, K. Beloborodov 122b,122a, K. Belotskiy ${ }^{112}$, N.L. Belyaev ${ }^{112}$, D. Benchekroun 35a, N. Benekos ${ }^{10}$, Y. Benhammou ${ }^{160}$, D.P. Benjamin ${ }^{6}$, M. Benoit ${ }^{54}$, J.R. Bensinger 26, S. Bentvelsen ${ }^{120}$, L. Beresford ${ }^{134}$, M. Beretta ${ }^{51}$, D. Berge ${ }^{19}$, E. Bergeaas Kuutmann ${ }^{171}$, N. Berger ${ }^{5}$, B. Bergmann ${ }^{141}$, L.J. Bergsten ${ }^{26}$, J. Beringer ${ }^{18}$, S. Berlendis ${ }^{7}$, G. Bernardi ${ }^{135}$, C. Bernius 
${ }^{152}$, F.U. Bernlochner ${ }^{24}$, T. Berry ${ }^{94}$, P. Berta ${ }^{100}$, C. Bertella ${ }^{15 a}$, A. Berthold ${ }^{48}$, I.A. Bertram ${ }^{90}$, O. Bessidskaia Bylund ${ }^{181}$, N. Besson ${ }^{144}$, A. Bethani ${ }^{101}$, S. Bethke ${ }^{115}$, A. Betti ${ }^{42}$, A.J. Bevan ${ }^{93}$, J. Beyer ${ }^{115}$, D.S. Bhattacharya ${ }^{176}$, P. Bhattarai ${ }^{26}$, R. Bi ${ }^{138}$, R.M. Bianchi ${ }^{138}$, O. Biebel ${ }^{114}$, D. Biedermann ${ }^{19}$, R. Bielski ${ }^{36}$, K. Bierwagen ${ }^{100}$, N.V. Biesuz ${ }^{72 a, 72 b}$, M. Biglietti ${ }^{75 a}$, T.R.V. Billoud ${ }^{110}$, M. Bindi ${ }^{53}$, A. Bingul ${ }^{12 d}$, C. Bini 73a,73b, S. Biondi 23b,23a, M. Birman ${ }^{179}$, T. Bisanz ${ }^{53}$, J.P. Biswal ${ }^{3}$, D. Biswas ${ }^{180, j}$, A. Bitadze ${ }^{101}$, C. Bittrich ${ }^{48}$, K. Bjørke ${ }^{133}$, T. Blazek ${ }^{28 a}$, I. Bloch ${ }^{46}$, C. Blocker ${ }^{26}$, A. Blue 57, U. Blumenschein ${ }^{93}$, G.J. Bobbink ${ }^{120}$, V.S. Bobrovnikov 122b,122a, S.S. Bocchetta ${ }^{97}$, A. Bocci ${ }^{49}$, D. Boerner 46, D. Bogavac 14, A.G. Bogdanchikov 122b,122a, C. Bohm ${ }^{45 a}$, V. Boisvert ${ }^{94}$, P. Bokan ${ }^{53,171,53}$, T. Bold ${ }^{84 a}$, A.E. Bolz ${ }^{61 b}$, M. Bomben ${ }^{135}$, M. Bona ${ }^{93}$, J.S. Bonilla ${ }^{131}$, M. Boonekamp ${ }^{144}$, C.D. Booth ${ }^{94}$, H.M. Borecka-Bielska ${ }^{91}$, L.S. Borgna ${ }^{95}$, A. Borisov ${ }^{123}$, G. Borissov ${ }^{90}$, J. Bortfeldt ${ }^{36}$, D. Bortoletto ${ }^{134}$, D. Boscherini ${ }^{23 b}$,

M. Bosman 14, J.D. Bossio Sola ${ }^{104}$, K. Bouaouda ${ }^{35 a}$, J. Boudreau ${ }^{138}$, E.V. Bouhova-Thacker ${ }^{90}$, D. Boumediene ${ }^{38}$, S.K. Boutle ${ }^{57}$, A. Boveia ${ }^{127}$, J. Boyd ${ }^{36}$, D. Boye ${ }^{33 c}$, I.R. Boyko ${ }^{80}$, A.J. Bozson ${ }^{94}$, J. Bracinik ${ }^{21}$, N. Brahimi ${ }^{102}$, G. Brandt ${ }^{181}$, O. Brandt ${ }^{22}$, F. Braren ${ }^{46}$, B. Brau ${ }^{103}$, J.E. Brau ${ }^{131}$, W.D. Breaden Madden ${ }^{57}$, K. Brendlinger ${ }^{46}$, L. Brenner ${ }^{46}$, R. Brenner ${ }^{171}$, S. Bressler ${ }^{179}$, B. Brickwedde ${ }^{100}$, D.L. Briglin ${ }^{21}$, D. Britton ${ }^{57}$, D. Britzger ${ }^{115}$, I. Brock ${ }^{24}$, R. Brock ${ }^{107}$, G. Brooijmans ${ }^{39}$, W.K. Brooks ${ }^{146 d}$, E. Brost ${ }^{29}$, P.A. Bruckman de Renstrom ${ }^{85}$, D. Bruncko ${ }^{28 b}$, A. Bruni ${ }^{23 b}$, G. Bruni ${ }^{23 b}$, L.S. Bruni ${ }^{120}$, S. Bruno ${ }^{74 a, 74 b}$, M. Bruschi ${ }^{23 b}$, N. Bruscino ${ }^{73 a, 73 b}$, L. Bryngemark ${ }^{152}$, T. Buanes ${ }^{17}$, Q. Buat ${ }^{36}$, P. Buchholz ${ }^{150}$, A.G. Buckley ${ }^{57}$, I.A. Budagov ${ }^{80}$, M.K. Bugge ${ }^{133}$, F. Bührer ${ }^{52}$, O. Bulekov ${ }^{112}$, B.A. Bullard ${ }^{59}$, T.J. Burch ${ }^{121}$, S. Burdin ${ }^{91}$, C.D. Burgard ${ }^{120}$, A.M. Burger ${ }^{129}$, B. Burghgrave ${ }^{8}$, J.T.P. Burr ${ }^{46}$, C.D. Burton ${ }^{11}$, J.C. Burzynski ${ }^{103}$, V. Büscher ${ }^{100}$, E. Buschmann ${ }^{53}$, P.J. Bussey ${ }^{57}$, J.M. Butler ${ }^{25}$, C.M. Buttar 57, J.M. Butterworth ${ }^{95}$, P. Butti ${ }^{36}$, W. Buttinger 36, C.J. Buxo Vazquez 107, A. Buzatu 157, A.R. Buzykaev 122b,122a , G. Cabras ${ }^{23 b, 23 a}$, S. Cabrera Urbán ${ }^{173}$, D. Caforio ${ }^{56}$, H. Cai ${ }^{172}$, V.M.M. Cairo ${ }^{152}$, O. Cakir 4a, N. Calace ${ }^{36}$, P. Calafiura ${ }^{18}$, G. Calderini ${ }^{135}$, P. Calfayan ${ }^{66}$, G. Callea ${ }^{57}$, L.P. Caloba 81b, A. Caltabiano ${ }^{74 a, 74 b}$, S. Calvente Lopez ${ }^{99}$, D. Calvet ${ }^{38}$, S. Calvet ${ }^{38}$, T.P. Calvet ${ }^{154}$, M. Calvetti ${ }^{72 a, 72 b}$, R. Camacho Toro ${ }^{135}$, S. Camarda ${ }^{36}$, D. Camarero Munoz ${ }^{99}$, P. Camarri 74a,74b, M.T. Camerlingo ${ }^{75 a, 75 b}$, D. Cameron ${ }^{133}$, C. Camincher ${ }^{36}$, S. Campana ${ }^{36}$,

M. Campanelli ${ }^{95}$, A. Camplani ${ }^{40}$, A. Campoverde ${ }^{150}$, V. Canale ${ }^{70 a, 70 b}$, A. Canesse ${ }^{104}$, M. Cano Bret ${ }^{78}$, J. Cantero ${ }^{129}$, T. Cao ${ }^{160}$, Y. Cao ${ }^{172}$, M.D.M. Capeans Garrido ${ }^{36}$, M. Capua 41b,41a, R. Cardarelli ${ }^{74 a}$, F. Cardillo ${ }^{148}$, G. Carducci ${ }^{41 b, 41 a}$, I. Carli ${ }^{142}$, T. Carli ${ }^{36}$, G. Carlino ${ }^{70 a}$, B.T. Carlson ${ }^{138}$, E.M. Carlson ${ }^{175,167 a}$, L. Carminati 69a,69b, R.M.D. Carney ${ }^{152}$, S. Caron ${ }^{119}$, E. Carquin ${ }^{146 \mathrm{~d}}$, S. Carrá ${ }^{46}$, J.W.S. Carter ${ }^{166}$, T.M. Carter 50, M.P. Casado ${ }^{14, f}$, A.F. Casha ${ }^{166}$, F.L. Castillo ${ }^{173}$, L. Castillo Garcia ${ }^{14}$,

V. Castillo Gimenez ${ }^{173}$, N.F. Castro ${ }^{139 a, 139 e}$, A. Catinaccio ${ }^{36}$, J.R. Catmore ${ }^{133}$, A. Cattai 36, V. Cavaliere ${ }^{29}$, E. Cavallaro ${ }^{14}$, V. Cavasinni ${ }^{72 a, 72 b}$, E. Celebi ${ }^{12 b}$, F. Celli ${ }^{134}$,

L. Cerda Alberich ${ }^{173}$, K. Cerny ${ }^{130}$, A.S. Cerqueira ${ }^{81 a}$, A. Cerri ${ }^{155}$, L. Cerrito ${ }^{74 a, 74 b}$, F. Cerutti ${ }^{18}$, A. Cervelli ${ }^{23 b, 23 a}$, S.A. Cetin ${ }^{12 b}$, Z. Chadi ${ }^{35 a}$, D. Chakraborty ${ }^{121}$, J. Chan ${ }^{180}$, W.S. Chan ${ }^{120}$, W.Y. Chan ${ }^{91}$, J.D. Chapman ${ }^{32}$, B. Chargeishvili 158b, D.G. Charlton ${ }^{21}$, T.P. Charman ${ }^{93}$, C.C. Chau ${ }^{34}$, S. Che ${ }^{127}$, S. Chekanov ${ }^{6}$, S.V. Chekulaev ${ }^{167 a}$, G.A. Chelkov 80,ai, B. Chen ${ }^{79}$, C. Chen ${ }^{60 a}$, C.H. Chen ${ }^{79}$, H. Chen ${ }^{29}$, J. Chen ${ }^{60 a}$, J. Chen ${ }^{39}$, J. Chen ${ }^{26}$, S. Chen ${ }^{136}$, S.J. Chen ${ }^{15 c}$, X. Chen ${ }^{15 b}$, Y. Chen ${ }^{60 a}$, Y-H. Chen ${ }^{46}$, H.C. Cheng ${ }^{63 a}$, H.J. Cheng ${ }^{15 a}$, A. Cheplakov ${ }^{80}$, E. Cheremushkina ${ }^{123}$, R. Cherkaoui El Moursli ${ }^{35 e}$, E. Cheu ${ }^{7}$, K. Cheung ${ }^{64}$, T.J.A. Chevalérias ${ }^{144}$, L. Chevalier ${ }^{144}$, V. Chiarella ${ }^{51}$, G. Chiarelli ${ }^{72 a}$, G. Chiodini ${ }^{68 a}$, A.S. Chisholm ${ }^{21}$, A. Chitan ${ }^{27 b}$, I. Chiu ${ }^{162}$, Y.H. Chiu ${ }^{175}$, M.V. Chizhov ${ }^{80}$, K. Choi ${ }^{11}$, A.R. Chomont ${ }^{73 a}, 73 b$, S. Chouridou ${ }^{161}$, Y.S. Chow ${ }^{120}$, L.D. Christopher ${ }^{33 e}$, 
M.C. Chu ${ }^{63 a}$, X. Chu ${ }^{15 a, 15 d}$, J. Chudoba ${ }^{140}$, J.J. Chwastowski ${ }^{85}$, L. Chytka ${ }^{130}$, D. Cieri ${ }^{115}$, K.M. Ciesla ${ }^{85}$, D. Cinca ${ }^{47}$, V. Cindro ${ }^{92}$, I.A. Cioară ${ }^{27 b}$, A. Ciocio ${ }^{18}$, F. Cirotto 70a,70b, Z.H. Citron 179,k, M. Citterio 69a, D.A. Ciubotaru 27b, B.M. Ciungu ${ }^{166}$, A. Clark ${ }^{54}$, M.R. Clark ${ }^{39}$, P.J. Clark ${ }^{50}$, S.E. Clawson ${ }^{101}$, C. Clement 45a,45b, Y. Coadou ${ }^{102}$, M. Cobal 67a,67c, A. Coccaro 55b, J. Cochran ${ }^{79}$, R. Coelho Lopes De Sa ${ }^{103}$, H. Cohen ${ }^{160}$, A.E.C. Coimbra ${ }^{36}$, B. Cole ${ }^{39}$, A.P. Colijn ${ }^{120}$, J. Collot ${ }^{58}$, P. Conde Muiño 139a,139h, S.H. Connell ${ }^{33 c}$, I.A. Connelly ${ }^{57}$, S. Constantinescu ${ }^{27 b}$, F. Conventi ${ }^{70 a, a o,}$ A.M. Cooper-Sarkar ${ }^{134}$, F. Cormier ${ }^{174}$, K.J.R. Cormier ${ }^{166}$, L.D. Corpe ${ }^{95}$, M. Corradi 73a,73b, J.G. Corrado ${ }^{125}$, E.E. Corrigan ${ }^{97}$, F. Corriveau 104,ad, M.J. Costa ${ }^{173}$, F. Costanza ${ }^{5}$, D. Costanzo ${ }^{148}$, G. Cowan ${ }^{94}$, J.W. Cowley ${ }^{32}$, J. Crane ${ }^{101}$, K. Cranmer ${ }^{125}$, S.J. Crawley ${ }^{57}$, R.A. Creager ${ }^{136}$, S. Crépé-Renaudin ${ }^{58}$, F. Crescioli ${ }^{135}$, M. Cristinziani ${ }^{24}$, V. Croft ${ }^{169}$, G. Crosetti ${ }^{41 \mathrm{~b}, 41 \mathrm{a}}$, A. Cueto ${ }^{5}$, T. Cuhadar Donszelmann ${ }^{170}$, A.R. Cukierman ${ }^{152}$, W.R. Cunningham ${ }^{57}$, S. Czekierda ${ }^{85}$, P. Czodrowski ${ }^{36}$, M.M. Czurylo ${ }^{61 b}$,

M.J. Da Cunha Sargedas De Sousa ${ }^{60 b}$, J.V. Da Fonseca Pinto ${ }^{81 b}$, C. Da Via ${ }^{101}$, W. Dabrowski ${ }^{84 a}$, F. Dachs ${ }^{36}$, T. Dado ${ }^{28 a}$, S. Dahbi ${ }^{33 e}$, T. Dai ${ }^{106}$, C. Dallapiccola ${ }^{103}$, M. Dam ${ }^{40}$, G. D'amen ${ }^{29}$, V. D'Amico ${ }^{75 a, 75 b}$, J. Damp ${ }^{100}$, J.R. Dandoy 136, M.F. Daneri ${ }^{30}$, N.S. Dann ${ }^{101}$, M. Danninger ${ }^{151}$, V. Dao ${ }^{36}$, G. Darbo ${ }^{55 b}$, O. Dartsi ${ }^{5}$, A. Dattagupta ${ }^{131}$, T. Daubney ${ }^{46}$, S. D'Auria ${ }^{69 a, 69 b}$, C. David ${ }^{167 b}$, T. Davidek ${ }^{142}$, D.R. Davis ${ }^{49}$, I. Dawson ${ }^{148}$, K. De ${ }^{8}$, R. De Asmundis ${ }^{70 a}$, M. De Beurs ${ }^{120}$, S. De Castro ${ }^{23 b, 23 a}$, S. De Cecco ${ }^{73 a, 73 b}$, N. De Groot ${ }^{119}$, P. de Jong ${ }^{120}$, H. De la Torre ${ }^{107}$, A. De Maria ${ }^{15 c}$, D. De Pedis ${ }^{73 a}$, A. De Salvo ${ }^{73 a}$, U. De Sanctis ${ }^{74 a, 74 b}$, M. De Santis ${ }^{74 a, 74 b}$, A. De Santo ${ }^{155}$,

J.B. De Vivie De Regie ${ }^{65}$, C. Debenedetti ${ }^{145}$, D.V. Dedovich ${ }^{80}$, A.M. Deiana ${ }^{42}$, J. Del Peso ${ }^{99}$, Y. Delabat Diaz ${ }^{46}$, D. Delgove ${ }^{65}$, F. Deliot ${ }^{144, q}$, C.M. Delitzsch ${ }^{7}$, M. Della Pietra ${ }^{70 a, 70 b}$, D. Della Volpe ${ }^{54}$, A. Dell'Acqua ${ }^{36}$, L. Dell'Asta ${ }^{74 a, 74 b}$, M. Delmastro ${ }^{5}$, C. Delporte ${ }^{65}$, P.A. Delsart ${ }^{58}$, D.A. DeMarco ${ }^{166}$, S. Demers ${ }^{182}$, M. Demichev ${ }^{80}$, G. Demontigny ${ }^{110}$, S.P. Denisov ${ }^{123}$, L. D'Eramo ${ }^{121}$, D. Derendarz ${ }^{85}$, J.E. Derkaoui ${ }^{35 d}$, F. Derue ${ }^{135}$, P. Dervan ${ }^{91}$, K. Desch ${ }^{24}$, C. Deterre ${ }^{46}$, K. Dette ${ }^{166}$, C. Deutsch ${ }^{24}$, M.R. Devesa ${ }^{30}$, P.O. Deviveiros ${ }^{36}$, F.A. Di Bello ${ }^{73 a, 73 b}$, A. Di Ciaccio ${ }^{74 a, 74 b}$, L. Di Ciaccio ${ }^{5}$, W.K. Di Clemente ${ }^{136}$, C. Di Donato 70a,70b, A. Di Girolamo ${ }^{36}$, G. Di Gregorio 72a,72b, B. Di Micco 75a,75b, R. Di Nardo ${ }^{75 a, 75 b}$, K.F. Di Petrillo ${ }^{59}$, R. Di Sipio ${ }^{166}$, C. Diaconu ${ }^{102}$, F.A. Dias ${ }^{40}$, T. Dias Do Vale ${ }^{139 a}$, M.A. Diaz ${ }^{146 a}$, F.G. Diaz Capriles ${ }^{24}$, J. Dickinson ${ }^{18}$, E.B. Diehl ${ }^{106}$, J. Dietrich ${ }^{19}$, S. Díez Cornell ${ }^{46}$, A. Dimitrievska ${ }^{18}$, W. Ding ${ }^{15 b}$, J. Dingfelder ${ }^{24}$, F. Dittus 36, F. Djama ${ }^{102}$, T. Djobava ${ }^{158 b}$, J.I. Djuvsland ${ }^{17}$, M.A.B. Do Vale ${ }^{81 \mathrm{c}}$, M. Dobre ${ }^{27 b}$, D. Dodsworth ${ }^{26}$, C. Doglioni ${ }^{97}$, J. Dolejsi ${ }^{142}$, Z. Dolezal ${ }^{142}$, M. Donadelli ${ }^{81 d}$, B. Dong ${ }^{60 c}$, J. Donini ${ }^{38}$, A. D'onofrio ${ }^{15 c}$, M. D'Onofrio ${ }^{91}$, J. Dopke ${ }^{143}$, A. Doria ${ }^{70 a}$, M.T. Dova ${ }^{89}$, A.T. Doyle ${ }^{57}$, E. Drechsler ${ }^{151}$, E. Dreyer ${ }^{151}$, T. Dreyer ${ }^{53}$, A.S. Drobac ${ }^{169}$, D. Du ${ }^{60 b}$, T.A. du Pree ${ }^{120}$, Y. Duan ${ }^{60 b}$, F. Dubinin ${ }^{111}$, M. Dubovsky ${ }^{28 a}$, A. Dubreuil ${ }^{54}$, E. Duchovni ${ }^{179}$, G. Duckeck ${ }^{114}$, O.A. Ducu ${ }^{27 b}$, D. Duda ${ }^{115}$, A. Dudarev ${ }^{36}$, A.C. Dudder ${ }^{100}$, E.M. Duffield ${ }^{18}$, L. Duflot ${ }^{65}$, M. Dührssen ${ }^{36}$, C. Dülsen ${ }^{181}$, M. Dumancic ${ }^{179}$, A.E. Dumitriu 27b, A.K. Duncan ${ }^{57}$, M. Dunford ${ }^{61 a}$, A. Duperrin ${ }^{102}$, H. Duran Yildiz ${ }^{4 a}$, M. Düren ${ }^{56}$, A. Durglishvili ${ }^{158 b}$, D. Duschinger ${ }^{48}$, B. Dutta ${ }^{46}$, D. Duvnjak ${ }^{1}$, G.I. Dyckes 136, M. Dyndal ${ }^{36}$, S. Dysch ${ }^{101}$, B.S. Dziedzic ${ }^{85}$, K.M. Ecker ${ }^{115}$, M.G. Eggleston ${ }^{49}$,

T. Eifert ${ }^{8}$, G. Eigen ${ }^{17}$, K. Einsweiler ${ }^{18}$, T. Ekelof ${ }^{171}$, H. El Jarrari ${ }^{35 e}$, R. El Kosseifi ${ }^{102}$, V. Ellajosyula ${ }^{171}$, M. Ellert ${ }^{171}$, F. Ellinghaus ${ }^{181}$, A.A. Elliot ${ }^{93}$, N. Ellis ${ }^{36}$, J. Elmsheuser 29, M. Elsing ${ }^{36}$, D. Emeliyanov ${ }^{143}$, A. Emerman ${ }^{39}$, Y. Enari ${ }^{162}$, M.B. Epland ${ }^{49}$, J. Erdmann ${ }^{47}$, A. Ereditato ${ }^{20}$, P.A. Erland ${ }^{85}$, M. Errenst ${ }^{36}$, M. Escalier ${ }^{65}$, C. Escobar ${ }^{173}$, O. Estrada Pastor ${ }^{173}$, E. Etzion ${ }^{160}$, H. Evans ${ }^{66}$, M.O. Evans ${ }^{155}$, A. Ezhilov ${ }^{137}$, F. Fabbri 57, L. Fabbri ${ }^{23 b, 23 a}$, V. Fabiani ${ }^{119}$, G. Facini ${ }^{177}$, R.M. Faisca Rodrigues Pereira ${ }^{139 a}$, 
R.M. Fakhrutdinov ${ }^{123}$, S. Falciano ${ }^{73 a}$, P.J. Falke ${ }^{24}$, S. Falke ${ }^{36}$, J. Faltova ${ }^{142}$, Y. Fang ${ }^{15 a}$, Y. Fang 15a, G. Fanourakis ${ }^{44}$, M. Fanti 69a,69b, M. Faraj 67a,67c,s, A. Farbin ${ }^{8}$, A. Farilla ${ }^{75 a}$, E.M. Farina ${ }^{71 a, 71 b}$, T. Farooque ${ }^{107}$, S.M. Farrington ${ }^{50}$, P. Farthouat ${ }^{36}$, F. Fassi ${ }^{35 e}$, P. Fassnacht ${ }^{36}$, D. Fassouliotis ${ }^{9}$, M. Faucci Giannelli ${ }^{50}$, W.J. Fawcett ${ }^{32}$, L. Fayard ${ }^{65}$, O.L. Fedin 137,p, W. Fedorko ${ }^{174}$, A. Fehr ${ }^{20}$, M. Feickert ${ }^{172}$, L. Feligioni ${ }^{102}$, A. Fell ${ }^{148}$, C. Feng ${ }^{60 b}$, M. Feng ${ }^{49}$, M.J. Fenton ${ }^{170}$, A.B. Fenyuk ${ }^{123}$, S.W. Ferguson ${ }^{43}$, J. Ferrando ${ }^{46}$, A. Ferrante ${ }^{172}$, A. Ferrari ${ }^{171}$, P. Ferrari ${ }^{120}$, R. Ferrari ${ }^{71 a}$, D.E. Ferreira de Lima ${ }^{61 b}$, A. Ferrer ${ }^{173}$, D. Ferrere ${ }^{54}$, C. Ferretti ${ }^{106}$, F. Fiedler ${ }^{100}$, A. Filipčič ${ }^{92}$, F. Filthaut ${ }^{119}$, K.D. Finelli ${ }^{25}$, M.C.N. Fiolhais ${ }^{139 a, 139 c, a}$, L. Fiorini ${ }^{173}$, F. Fischer ${ }^{114}$, W.C. Fisher ${ }^{107}$, I. Fleck $^{150}$, P. Fleischmann ${ }^{106}$, T. Flick ${ }^{181}$, B.M. Flierl ${ }^{114}$, L. Flores ${ }^{136}$, L.R. Flores Castillo 63a, F.M. Follega 76a,76b, N. Fomin ${ }^{17}$, J.H. Foo ${ }^{166}$, G.T. Forcolin 76a,76b, A. Formica ${ }^{144}$, F.A. Förster ${ }^{14}$, A.C. Forti ${ }^{101}$, E. Fortin ${ }^{102}$, M.G. Foti ${ }^{134}$, D. Fournier ${ }^{65}$, H. Fox ${ }^{90}$, P. Francavilla 72a,72b, S. Francescato ${ }^{73 a, 73 b}$, M. Franchini ${ }^{23 b, 23 a}$, S. Franchino ${ }^{61 a}$, D. Francis 36, L. Franco ${ }^{5}$, L. Franconi ${ }^{20}$, M. Franklin ${ }^{59}$, G. Frattari ${ }^{73 a, 73 b}$, A.N. Fray ${ }^{93}$, P.M. Freeman ${ }^{21}$, B. Freund ${ }^{110}$, W.S. Freund ${ }^{81 b}$, E.M. Freundlich 47, D.C. Frizzell ${ }^{128}$, D. Froidevaux ${ }^{36}$, J.A. Frost ${ }^{134}$, M. Fujimoto ${ }^{126}$, C. Fukunaga ${ }^{163}$, E. Fullana Torregrosa ${ }^{173}$, T. Fusayasu 116, J. Fuster ${ }^{173}$, A. Gabrielli 23b,23a, A. Gabrielli 18, S. Gadatsch ${ }^{54}$, P. Gadow ${ }^{115}$, G. Gagliardi 55b,55a, L.G. Gagnon ${ }^{110}$, B. Galhardo ${ }^{139 a}$, G.E. Gallardo ${ }^{134}$, E.J. Gallas ${ }^{134}$, B.J. Gallop ${ }^{143}$, G. Galster ${ }^{40}$, R. Gamboa Goni ${ }^{93}$, K.K. Gan ${ }^{127}$, S. Ganguly ${ }^{179}$, J. Gao ${ }^{60 a}$, Y. Gao ${ }^{50}$, Y.S. Gao ${ }^{31, m}$, F.M. Garay Walls ${ }^{146 a}$, C. García ${ }^{173}$, J.E. García Navarro ${ }^{173}$, J.A. García Pascual ${ }^{15 a}$, C. Garcia-Argos ${ }^{52}$, M. Garcia-Sciveres ${ }^{18}$, R.W. Gardner ${ }^{37}$, N. Garelli ${ }^{152}$, S. Gargiulo ${ }^{52}$, C.A. Garner ${ }^{166}$, V. Garonne ${ }^{133}$, S.J. Gasiorowski ${ }^{147}$, P. Gaspar ${ }^{81 b}$, A. Gaudiello ${ }^{55 b, 55 a}$, G. Gaudio ${ }^{71 a}$, I.L. Gavrilenko ${ }^{111}$, A. Gavrilyuk ${ }^{124}$, C. Gay ${ }^{174}$, G. Gaycken ${ }^{46}$, E.N. Gazis ${ }^{10}$, A.A. Geanta ${ }^{27 b}$, C.M. Gee ${ }^{145}$, C.N.P. Gee ${ }^{143}$, J. Geisen ${ }^{97}$, M. Geisen ${ }^{100}$, C. Gemme ${ }^{55 b}$, M.H. Genest ${ }^{58}$, C. Geng ${ }^{106}$, S. Gentile ${ }^{73 a, 73 b}$, S. George ${ }^{94}$, T. Geralis ${ }^{44}$, L.O. Gerlach ${ }^{53}$, P. Gessinger-Befurt ${ }^{100}$, G. Gessner ${ }^{47}$, S. Ghasemi ${ }^{150}$, M. Ghasemi Bostanabad ${ }^{175}$, M. Ghneimat ${ }^{150}$, A. Ghosh ${ }^{65}$, A. Ghosh ${ }^{78}$, B. Giacobbe ${ }^{23 b}$, S. Giagu 73a,73b, N. Giangiacomi 23b,23a, P. Giannetti 72a, A. Giannini ${ }^{70 a, 70 b,}$ G. Giannini ${ }^{14}$, S.M. Gibson ${ }^{94}$, M. Gignac ${ }^{145}$, D. Gillberg ${ }^{34}$, G. Gilles ${ }^{181}$, D.M. Gingrich 3,an, M.P. Giordani 67a,67c, P.F. Giraud ${ }^{144}$, G. Giugliarelli 67a,67c, D. Giugni 69a, F. Giuli 74a,74b, S. Gkaitatzis ${ }^{161}$, I. Gkialas ${ }^{9, h}$, E.L. Gkougkousis ${ }^{14}$, P. Gkountoumis ${ }^{10}$, L.K. Gladilin ${ }^{113}$, C. Glasman ${ }^{99}$, J. Glatzer ${ }^{14}$, P.C.F. Glaysher ${ }^{46}$, A. Glazov ${ }^{46}$, G.R. Gledhill ${ }^{131}$, I. Gnesi 41b,b, M. Goblirsch-Kolb 26, D. Godin ${ }^{110}$, S. Goldfarb 105, T. Golling ${ }^{54}$, D. Golubkov 123, A. Gomes 139a,139b, R. Goncalves Gama ${ }^{53}$, R. Gonçalo 139a, G. Gonella ${ }^{131}$, L. Gonella ${ }^{21}$, A. Gongadze ${ }^{80}$, F. Gonnella ${ }^{21}$, J.L. Gonski ${ }^{39}$, S. González de la Hoz ${ }^{173}$, S. Gonzalez Fernandez ${ }^{14}$, C. Gonzalez Renteria ${ }^{18}$, R. Gonzalez Suarez ${ }^{171}$, S. Gonzalez-Sevilla 54 , G.R. Gonzalvo Rodriguez ${ }^{173}$, L. Goossens ${ }^{36}$, N.A. Gorasia ${ }^{21}$, P.A. Gorbounov ${ }^{124}$, H.A. Gordon ${ }^{29}$, B. Gorini ${ }^{36}$, E. Gorini 68a,68b, A. Gorišek ${ }^{92}$, A.T. Goshaw ${ }^{49}$, M.I. Gostkin ${ }^{80}$, C.A. Gottardo ${ }^{119}$, M. Gouighri ${ }^{35 b}$, A.G. Goussiou ${ }^{147}$, N. Govender 33c, C. Goy ${ }^{5}$, E. Gozani ${ }^{159}$, I. Grabowska-Bold ${ }^{84 a}$, E.C. Graham ${ }^{91}$, J. Gramling ${ }^{170}$, E. Gramstad ${ }^{133}$, S. Grancagnolo ${ }^{19}$, M. Grandi ${ }^{155}$, V. Gratchev ${ }^{137}$, P.M. Gravila ${ }^{27 f}$, F.G. Gravili 68a,68b, C. Gray ${ }^{57}$, H.M. Gray ${ }^{18}$, C. Grefe ${ }^{24}$, K. Gregersen ${ }^{97}$, I.M. Gregor ${ }^{46}$, P. Grenier ${ }^{152}$, K. Grevtsov ${ }^{46}$, C. Grieco ${ }^{14}$, N.A. Grieser ${ }^{128}$, A.A. Grillo ${ }^{145}$, K. Grimm ${ }^{31,1}$, S. Grinstein 14,y, J.-F. Grivaz ${ }^{65}$, S. Groh ${ }^{100}$, E. Gross ${ }^{179}$, J. Grosse-Knetter ${ }^{53}$, Z.J. Grout ${ }^{95}$, C. Grud ${ }^{106}$, A. Grummer ${ }^{118}$, J.C. Grundy ${ }^{134}$, L. Guan ${ }^{106}$, W. Guan ${ }^{180}$, C. Gubbels ${ }^{174}$, J. Guenther ${ }^{36}$, A. Guerguichon ${ }^{65}$, J.G.R. Guerrero Rojas ${ }^{173}$, F. Guescini ${ }^{115}$, D. Guest ${ }^{170}$, R. Gugel ${ }^{52}$, T. Guillemin ${ }^{5}$, S. Guindon ${ }^{36}$, U. Gul ${ }^{57}$, J. Guo ${ }^{60 c}$, W. Guo ${ }^{106}$, Y. Guo ${ }^{60 a}$, Z. Guo ${ }^{102}$, R. Gupta ${ }^{46}$, S. Gurbuz ${ }^{12 c}$, G. Gustavino ${ }^{128}$, M. Guth ${ }^{52}$, P. Gutierrez ${ }^{128}$, C. Gutschow ${ }^{95}$, 
C. Guyot ${ }^{144}$, C. Gwenlan ${ }^{134}$, C.B. Gwilliam ${ }^{91}$, E.S. Haaland ${ }^{133}$, A. Haas ${ }^{125}$, C. Haber ${ }^{18}$, H.K. Hadavand ${ }^{8}$, A. Hadef ${ }^{60 a}$, M. Haleem ${ }^{176}$, J. Haley ${ }^{129}$, J.J. Hall ${ }^{148}$, G. Halladjian ${ }^{107}$, G.D. Hallewell ${ }^{102}$, K. Hamacher ${ }^{181}$, P. Hamal ${ }^{130}$, K. Hamano ${ }^{175}$, H. Hamdaoui ${ }^{35 e}$, M. Hamer ${ }^{24}$, G.N. Hamity ${ }^{50}$, K. Han ${ }^{60 a, x}$, L. Han ${ }^{60 a}$, S. Han ${ }^{18}$, Y.F. Han ${ }^{166}$, K. Hanagaki ${ }^{82, \mathrm{v}}$, M. Hance ${ }^{145}$, D.M. Handl ${ }^{114}$, B. Haney ${ }^{136}$, M.D. Hank ${ }^{37}$, R. Hankache ${ }^{135}$,

E. Hansen ${ }^{97}$, J.B. Hansen ${ }^{40}$, J.D. Hansen ${ }^{40}$, M.C. Hansen ${ }^{24}$, P.H. Hansen ${ }^{40}$, E.C. Hanson 101, K. Hara ${ }^{168}$, T. Harenberg ${ }^{181}$, S. Harkusha ${ }^{108}$, P.F. Harrison ${ }^{177}$, N.M. Hartman ${ }^{152}$, N.M. Hartmann ${ }^{114}$, Y. Hasegawa ${ }^{149}$, A. Hasib ${ }^{50}$, S. Hassani ${ }^{144}$, S. Haug ${ }^{20}$, R. Hauser ${ }^{107}$, L.B. Havener ${ }^{39}$, M. Havranek ${ }^{141}$, C.M. Hawkes ${ }^{21}$, R.J. Hawkings ${ }^{36}$, S. Hayashida ${ }^{117}$, D. Hayden ${ }^{107}$, C. Hayes ${ }^{106}$, R.L. Hayes ${ }^{174}$, C.P. Hays ${ }^{134}$, J.M. Hays ${ }^{93}$, H.S. Hayward ${ }^{91}$, S.J. Haywood ${ }^{143}$, F. He ${ }^{60 a}$, M.P. Heath ${ }^{50}$, V. Hedberg ${ }^{97}$, S. Heer ${ }^{24}$, A.L. Heggelund ${ }^{133}$, K.K. Heidegger ${ }^{52}$, W.D. Heidorn ${ }^{79}$, J. Heilman ${ }^{34}$, S. Heim ${ }^{46}$, T. Heim ${ }^{18}$, B. Heinemann 46,al, J.J. Heinrich ${ }^{131}$, L. Heinrich ${ }^{36}$, J. Hejbal ${ }^{140}$, L. Helary ${ }^{61 b}$, A. Held ${ }^{125}$, S. Hellesund ${ }^{133}$, C.M. Helling ${ }^{145}$, S. Hellman ${ }^{45 a, 45 b}$, C. Helsens ${ }^{36}$, R.C.W. Henderson 90, Y. Heng ${ }^{180}$, L. Henkelmann ${ }^{32}$, A.M. Henriques Correia ${ }^{36}$, H. Herde ${ }^{26}$, Y. Hernández Jiménez ${ }^{33 e}$, H. Herr ${ }^{100}$, M.G. Herrmann ${ }^{114}$, T. Herrmann ${ }^{48}$, G. Herten ${ }^{52}$, R. Hertenberger ${ }^{114}$, L. Hervas ${ }^{36}$, T.C. Herwig ${ }^{136}$, G.G. Hesketh ${ }^{95}$, N.P. Hessey ${ }^{167 a}$, H. Hibi ${ }^{83}$, A. Higashida 162, S. Higashino ${ }^{82}$, E. Higón-Rodriguez ${ }^{173}$, K. Hildebrand ${ }^{37}$, J.C. Hill ${ }^{32}$, K.K. Hill ${ }^{29}$, K.H. Hiller ${ }^{46}$, S.J. Hillier ${ }^{21}$, M. Hils ${ }^{48}$, I. Hinchliffe ${ }^{18}$, F. Hinterkeuser ${ }^{24}$, M. Hirose ${ }^{132}$, S. Hirose ${ }^{52}$, D. Hirschbuehl ${ }^{181}$, B. Hiti ${ }^{92}$, O. Hladik ${ }^{140}$, D.R. Hlaluku 33e, J. Hobbs ${ }^{154}$, N. Hod ${ }^{179}$, M.C. Hodgkinson ${ }^{148}$, A. Hoecker ${ }^{36}$, D. Hohn ${ }^{52}$, D. Hohov ${ }^{65}$, T. Holm ${ }^{24}$, T.R. Holmes ${ }^{37}$, M. Holzbock ${ }^{114}$, L.B.A.H. Hommels ${ }^{32}$, T.M. Hong ${ }^{138}$, J.C. Honig ${ }^{52}$, A. Hönle ${ }^{115}$, B.H. Hooberman ${ }^{172}$, W.H. Hopkins ${ }^{6}$, Y. Horii ${ }^{117}$, P. Horn ${ }^{48}$, L.A. Horyn ${ }^{37}$, S. Hou ${ }^{157}$, A. Hoummada ${ }^{35 a}$, J. Howarth ${ }^{57}$, J. Hoya ${ }^{89}$, M. Hrabovsky ${ }^{130}$, J. Hrdinka ${ }^{77}$, I. Hristova ${ }^{19}$, J. Hrivnac ${ }^{65}$, A. Hrynevich ${ }^{109}$, T. Hryn'ova ${ }^{5}$, P.J. Hsu ${ }^{64}$, S.-C. Hsu ${ }^{147}$, Q. $\mathrm{Hu}^{29}$, S. Hu ${ }^{60 c}$, Y.F. Hu ${ }^{15 a, 15 d}$, D.P. Huang ${ }^{95}$, Y. Huang ${ }^{60 a}$, Y. Huang ${ }^{15 a}$, Z. Hubacek ${ }^{141}$, F. Hubaut ${ }^{102}$, M. Huebner ${ }^{24}$, F. Huegging ${ }^{24}$, T.B. Huffman ${ }^{134}$, M. Huhtinen ${ }^{36}$,

R.F.H. Hunter ${ }^{34}$, P. Huo ${ }^{154}$, N. Huseynov ${ }^{80, a e}$, J. Huston ${ }^{107}$, J. Huth 59, R. Hyneman ${ }^{106}$, S. Hyrych ${ }^{28 a}$, G. Iacobucci ${ }^{54}$, G. Iakovidis ${ }^{29}$, I. Ibragimov ${ }^{150}$, L. Iconomidou-Fayard ${ }^{65}$, P. Iengo ${ }^{36}$, R. Ignazzi ${ }^{40}$, O. Igonkina ${ }^{120, a a, *}$, R. Iguchi ${ }^{162}$, T. Iizawa ${ }^{54}$, Y. Ikegami ${ }^{82}$, M. Ikeno ${ }^{82}$, D. Iliadis ${ }^{161}$, N. Ilic ${ }^{119,166, a d}$, F. Iltzsche ${ }^{48}$, H. Imam ${ }^{35 a}$, G. Introzzi ${ }^{71 a, 71 b}$ M. Iodice ${ }^{75 a}$, K. Iordanidou ${ }^{167 a}$, V. Ippolito ${ }^{73 a}, 73 b$, M.F. Isacson ${ }^{171}$, M. Ishino ${ }^{162}$, W. Islam ${ }^{129}$, C. Issever ${ }^{19,46}$, S. Istin ${ }^{159}$, F. Ito ${ }^{168}$, J.M. Iturbe Ponce ${ }^{63 a}$, R. Iuppa ${ }^{76 a, 76 b}$, A. Ivina ${ }^{179}$, H. Iwasaki ${ }^{82}$, J.M. Izen ${ }^{43}$, V. Izzo ${ }^{70 a}$, P. Jacka ${ }^{140}$, P. Jackson ${ }^{1}$, R.M. Jacobs ${ }^{46}$, B.P. Jaeger ${ }^{151}$, V. Jain ${ }^{2}$, G. Jäkel ${ }^{181}$, K.B. Jakobi ${ }^{100}$, K. Jakobs ${ }^{52}$, T. Jakoubek ${ }^{179}$, J. Jamieson ${ }^{57}$, K.W. Janas ${ }^{84 a}$, R. Jansky ${ }^{54}$, M. Janus ${ }^{53}$, P.A. Janus ${ }^{84 a}$, G. Jarlskog ${ }^{97}$, A.E. Jaspan ${ }^{91}$, N. Javadov ${ }^{80, a e}$, T. Javůrek ${ }^{36}$, M. Javurkova ${ }^{103}$, F. Jeanneau ${ }^{144}$, L. Jeanty ${ }^{131}$, J. Jejelava ${ }^{158 a}$, A. Jelinskas ${ }^{177}$, P. Jenni ${ }^{52, c}$, N. Jeong ${ }^{46}$, S. Jézéquel ${ }^{5}$, H. Ji ${ }^{180}$, J. Jia ${ }^{154}$, H. Jiang ${ }^{79}$, Y. Jiang ${ }^{60 a}$, Z. Jiang ${ }^{152}$, S. Jiggins ${ }^{52}$, F.A. Jimenez Morales ${ }^{38}$, J. Jimenez Pena ${ }^{115}$, S. Jin ${ }^{15 c}$, A. Jinaru ${ }^{27 b}$, O. Jinnouchi ${ }^{164}$, H. Jivan ${ }^{33 e}$, P. Johansson ${ }^{148}$, K.A. Johns ${ }^{7}$, C.A. Johnson ${ }^{66}$, R.W.L. Jones ${ }^{90}$, S.D. Jones ${ }^{155}$, S. Jones ${ }^{7}$, T.J. Jones ${ }^{91}$, J. Jongmanns ${ }^{61 a}$, P.M. Jorge ${ }^{139 a}$, J. Jovicevic ${ }^{36}$, X. Ju ${ }^{18}$, J.J. Junggeburth ${ }^{115}$, A. Juste Rozas ${ }^{14, y}$, A. Kaczmarska ${ }^{85}$, M. Kado ${ }^{73 a, 73 b}$, H. Kagan ${ }^{127}$, M. Kagan ${ }^{152}$, A. Kahn ${ }^{39}$, C. Kahra ${ }^{100}$, T. Kaji ${ }^{178}$, E. Kajomovitz ${ }^{159}$, C.W. Kalderon ${ }^{29}$, A. Kaluza ${ }^{100}$, A. Kamenshchikov ${ }^{123}$, M. Kaneda ${ }^{162}$, N.J. Kang ${ }^{145}$, S. Kang ${ }^{79}$, Y. Kano ${ }^{117}$, J. Kanzaki ${ }^{82}$, L.S. Kaplan ${ }^{180}$, D. Kar ${ }^{33 e}$, K. Karava ${ }^{134}$, M.J. Kareem ${ }^{167 b}$, I. Karkanias ${ }^{161}$, S.N. Karpov ${ }^{80}$, Z.M. Karpova ${ }^{80}$, V. Kartvelishvili ${ }^{90}$, A.N. Karyukhin ${ }^{123}$, A. Kastanas 45a,45b, C. Kato ${ }^{60 d, 60 c}$, J. Katzy ${ }^{46}$, K. Kawade ${ }^{149}$, K. Kawagoe ${ }^{88}$, T. Kawaguchi ${ }^{117}$, 
T. Kawamoto ${ }^{144}$, G. Kawamura ${ }^{53}$, E.F. Kay ${ }^{175}$, S. Kazakos ${ }^{14}$, V.F. Kazanin ${ }^{122 b, 122 a}$, R. Keeler ${ }^{175}$, R. Kehoe ${ }^{42}$, J.S. Keller ${ }^{34}$, E. Kellermann ${ }^{97}$, D. Kelsey ${ }^{155}$, J.J. Kempster ${ }^{21}$, J. Kendrick ${ }^{21}$, K.E. Kennedy ${ }^{39}$, O. Kepka ${ }^{140}$, S. Kersten ${ }^{181}$, B.P. Kerševan ${ }^{92}$, S. Ketabchi Haghighat ${ }^{166}$, M. Khader ${ }^{172}$, F. Khalil-Zada ${ }^{13}$, M. Khandoga ${ }^{144}$, A. Khanov 129, A.G. Kharlamov 122b,122a, T. Kharlamova 122b,122a, E.E. Khoda ${ }^{174}$, A. Khodinov ${ }^{165}$, T.J. Khoo ${ }^{54}$, G. Khoriauli ${ }^{176}$, E. Khramov ${ }^{80}$, J. Khubua ${ }^{158 b}$, S. Kido ${ }^{83}$, M. Kiehn ${ }^{54}$, C.R. Kilby ${ }^{94}$, E. Kim ${ }^{164}$, Y.K. Kim ${ }^{37}$, N. Kimura ${ }^{95}$, O.M. Kind ${ }^{19}$, B.T. King ${ }^{91, *}$, D. Kirchmeier ${ }^{48}$, J. Kirk ${ }^{143}$, A.E. Kiryunin ${ }^{115}$, T. Kishimoto ${ }^{162}$, D.P. Kisliuk ${ }^{166}$, V. Kitali ${ }^{46}$, C. Kitsaki ${ }^{10}$, O. Kivernyk ${ }^{24}$, T. Klapdor-Kleingrothaus ${ }^{52}$, M. Klassen ${ }^{61 a}$, C. Klein ${ }^{34}$, M.H. Klein ${ }^{106}$, M. Klein ${ }^{91}$, U. Klein ${ }^{91}$, K. Kleinknecht ${ }^{100}$, P. Klimek ${ }^{121}$, A. Klimentov ${ }^{29}$, T. Klingl ${ }^{24}$, T. Klioutchnikova ${ }^{36}$, F.F. Klitzner ${ }^{114}$, P. Kluit ${ }^{120}$, S. Kluth ${ }^{115}$, E. Kneringer 77, E.B.F.G. Knoops ${ }^{102}$, A. Knue ${ }^{52}$, D. Kobayashi ${ }^{88}$, T. Kobayashi ${ }^{162}$, M. Kobel ${ }^{48}$, M. Kocian ${ }^{152}$, T. Kodama ${ }^{162}$, P. Kodys ${ }^{142}$, D.M. Koeck ${ }^{155}$, P.T. Koenig ${ }^{24}$, T. Koffas ${ }^{34}$, N.M. Köhler ${ }^{36}$, M. Kolb ${ }^{144}$, I. Koletsou ${ }^{5}$, T. Komarek ${ }^{130}$, T. Kondo ${ }^{82}$, K. Köneke ${ }^{52}$, A.X.Y. Kong ${ }^{1}$, A.C. König ${ }^{119}$, T. Kono ${ }^{126}$, V. Konstantinides ${ }^{95}$, N. Konstantinidis ${ }^{95}$, B. Konya ${ }^{97}$, R. Kopeliansky ${ }^{66}$, S. Koperny ${ }^{84 a}$, K. Korcyl ${ }^{85}$, K. Kordas ${ }^{161}$, G. Koren ${ }^{160}$, A. Korn ${ }^{95}$, I. Korolkov ${ }^{14}$, E.V. Korolkova ${ }^{148}$, N. Korotkova ${ }^{113}$, O. Kortner ${ }^{115}$, S. Kortner ${ }^{115}$, V.V. Kostyukhin ${ }^{148,165}$, A. Kotsokechagia ${ }^{65}$, A. Kotwal ${ }^{49}$, A. Koulouris ${ }^{10}$,

A. Kourkoumeli-Charalampidi ${ }^{71 a, 71 b}$, C. Kourkoumelis ${ }^{9}$, E. Kourlitis ${ }^{6}$, V. Kouskoura ${ }^{29}$, A.B. Kowalewska ${ }^{85}$, R. Kowalewski ${ }^{175}$, W. Kozanecki ${ }^{101}$, A.S. Kozhin ${ }^{123}$, V.A. Kramarenko 113, G. Kramberger 92, D. Krasnopevtsev 60a , M.W. Krasny ${ }^{135}$, A. Krasznahorkay ${ }^{36}$, D. Krauss ${ }^{115}$, J.A. Kremer ${ }^{100}$, J. Kretzschmar ${ }^{91}$, P. Krieger ${ }^{166}$, F. Krieter ${ }^{114}$, A. Krishnan 61b, K. Krizka ${ }^{18}$, K. Kroeninger ${ }^{47}$, H. Kroha ${ }^{115}$, J. Kroll ${ }^{140}$, J. Kroll 136, K.S. Krowpman 107, U. Kruchonak ${ }^{80}$, H. Krüger ${ }^{24}$, N. Krumnack ${ }^{79}$, M.C. Kruse ${ }^{49}$, J.A. Krzysiak ${ }^{85}$, T. Kubota ${ }^{105}$, O. Kuchinskaia ${ }^{165}$, S. Kuday ${ }^{4 b}$, J.T. Kuechler ${ }^{46}$, S. Kuehn ${ }^{36}$, A. Kugel ${ }^{61 a}$, T. Kuhl ${ }^{46}$, V. Kukhtin ${ }^{80}$, Y. Kulchitsky ${ }^{108, a g}$, S. Kuleshov ${ }^{146 b}$, Y.P. Kulinich ${ }^{172}$, M. Kuna ${ }^{58}$, T. Kunigo ${ }^{86}$, A. Kupco ${ }^{140}$, T. Kupfer ${ }^{47}$, O. Kuprash ${ }^{52}$, H. Kurashige ${ }^{83}$,

L.L. Kurchaninov 167a, Y.A. Kurochkin ${ }^{108}$, A. Kurova ${ }^{112}$, M.G. Kurth 15a,15d, E.S. Kuwertz 36, M. Kuze ${ }^{164}$, A.K. Kvam ${ }^{147}$, J. Kvita ${ }^{130}$, T. Kwan ${ }^{104}$, L. La Rotonda 41b,41a,

F. La Ruffa ${ }^{41 b, 41 a}$, C. Lacasta ${ }^{173}$, F. Lacava ${ }^{73 a, 73 b}$, D.P.J. Lack ${ }^{101}$, H. Lacker ${ }^{19}$, D. Lacour ${ }^{135}$, E. Ladygin ${ }^{80}$, R. Lafaye ${ }^{5}$, B. Laforge ${ }^{135}$, T. Lagouri ${ }^{146 b}$, S. Lai ${ }^{53}$, I.K. Lakomiec ${ }^{84 a}$, J.E. Lambert ${ }^{128}$, S. Lammers ${ }^{66}$, W. Lampl ${ }^{7}$, C. Lampoudis ${ }^{161}$, E. Lançon ${ }^{29}$, U. Landgraf 52 , M.P.J. Landon ${ }^{93}$, M.C. Lanfermann ${ }^{54}$, V.S. Lang ${ }^{52}$, J.C. Lange ${ }^{53}$, R.J. Langenberg ${ }^{103}$, A.J. Lankford ${ }^{170}$, F. Lanni ${ }^{29}$, K. Lantzsch ${ }^{24}$, A. Lanza ${ }^{71 a}$, A. Lapertosa 55b,55a, S. Laplace 135, J.F. Laporte ${ }^{144}$, T. Lari 69a , F. Lasagni Manghi 23b,23a, M. Lassnig ${ }^{36}$, T.S. Lau ${ }^{63 a}$, A. Laudrain ${ }^{65}$, A. Laurier ${ }^{34}$, M. Lavorgna ${ }^{70 a, 70 b}$, S.D. Lawlor ${ }^{94}$, M. Lazzaroni 69a,69b, B. Le ${ }^{101}$, E. Le Guirriec ${ }^{102}$, A. Lebedev ${ }^{79}$, M. LeBlanc ${ }^{7}$, T. LeCompte ${ }^{6}$, F. Ledroit-Guillon ${ }^{58}$, A.C.A. Lee ${ }^{95}$, C.A. Lee ${ }^{29}$, G.R. Lee ${ }^{17}$, L. Lee ${ }^{59}$, S.C. Lee ${ }^{157}$, S. Lee ${ }^{79}$, B. Lefebvre 167a, H.P. Lefebvre ${ }^{94}$, M. Lefebvre ${ }^{175}$, C. Leggett ${ }^{18}$, K. Lehmann ${ }^{151}$, N. Lehmann ${ }^{20}$, G. Lehmann Miotto ${ }^{36}$, W.A. Leight ${ }^{46}$, A. Leisos ${ }^{161, w}$, M.A.L. Leite ${ }^{81 d}$, C.E. Leitgeb ${ }^{114}$, R. Leitner ${ }^{142}$, D. Lellouch ${ }^{179, *}$, K.J.C. Leney ${ }^{42}$, T. Lenz ${ }^{24}$, R. Leone ${ }^{7}$, S. Leone ${ }^{72 a}$, C. Leonidopoulos ${ }^{50}$, A. Leopold ${ }^{135}$, C. Leroy ${ }^{110}$, R. Les ${ }^{166}$, C.G. Lester ${ }^{32}$, M. Levchenko ${ }^{137}$, J. Levêque ${ }^{5}$, D. Levin ${ }^{106}$, L.J. Levinson ${ }^{179}$, D.J. Lewis ${ }^{21}$, B. Li ${ }^{15 b}$, B. Li ${ }^{106}$, C-Q. Li 60a, F. Li ${ }^{60 c}$, H. Li ${ }^{60 a}$, H. Li ${ }^{60 b}$, J. Li ${ }^{60 c}$, K. Li ${ }^{147}$, L. Li ${ }^{60 c}$, M. Li 15a,15d, Q. Li ${ }^{15 a, 15 d}$, Q.Y. Li ${ }^{60 a}, \mathrm{~S}_{\mathrm{Li}}{ }^{60 \mathrm{~d}, 60 \mathrm{c}}$, X. Li ${ }^{46}$, Y. Li ${ }^{46}$, Z. Li ${ }^{60 b}$, Z. Li ${ }^{104}$, Z. Liang 15a, M. Liberatore 46, B. Liberti ${ }^{74 a}$, A. Liblong ${ }^{166}$, K. Lie ${ }^{63 c}$, S. Lim ${ }^{29}$, C.Y. Lin ${ }^{32}$, K. Lin 107, T.H. Lin ${ }^{100}$, R.A. Linck ${ }^{66}$, R.E. Lindley ${ }^{7}$, J.H. Lindon ${ }^{21}$, A. Linss ${ }^{46}$, A.L. Lionti ${ }^{54}$, E. Lipeles ${ }^{136}$, A. Lipniacka ${ }^{17}$, T.M. Liss ${ }^{172, \text { am }}$, A. Lister ${ }^{174}$, J.D. Little ${ }^{8}$, B. Liu ${ }^{79}$, B.L. Liu ${ }^{6}$, H.B. Liu 
29, H. Liu 106, J.B. Liu ${ }^{60 a}$, J.K.K. Liu ${ }^{37}$, K. Liu ${ }^{60 d}$, M. Liu ${ }^{60 a}$, P. Liu 15a, Y. Liu ${ }^{46}$, Y. Liu ${ }^{15 a, 15 d}$, Y.L. Liu ${ }^{106}$, Y.W. Liu ${ }^{60 a}$, M. Livan ${ }^{71 a, 71 b}$, A. Lleres ${ }^{58}$, J. Llorente Merino ${ }^{151}$, S.L. Lloyd ${ }^{93}$, C.Y. Lo ${ }^{63 b}$, E.M. Lobodzinska ${ }^{46}$, P. Loch ${ }^{7}$, S. Loffredo ${ }^{74 a, 74 b}$, T. Lohse ${ }^{19}$, K. Lohwasser ${ }^{148}$, M. Lokajicek ${ }^{140}$, J.D. Long ${ }^{172}$, R.E. Long ${ }^{90}$, L. Longo ${ }^{36}$, K.A. Looper ${ }^{127}$, I. Lopez Paz ${ }^{101}$, A. Lopez Solis ${ }^{148}$, J. Lorenz ${ }^{114}$, N. Lorenzo Martinez ${ }^{5}$, A.M. Lory ${ }^{114}$, P.J. Lösel ${ }^{114}$, A. Lösle ${ }^{52}$, X. Lou ${ }^{46}$, X. Lou ${ }^{15 a}$, A. Lounis ${ }^{65}$, J. Love ${ }^{6}$, P.A. Love ${ }^{90}$, J.J. Lozano Bahilo ${ }^{173}$, M. Lu ${ }^{60 a}$, Y.J. Lu ${ }^{64}$, H.J. Lubatti ${ }^{147}$, C. Luci ${ }^{73 a}, 73 b$, A. Lucotte ${ }^{58}$, C. Luedtke ${ }^{52}$, F. Luehring ${ }^{66}$, I. Luise ${ }^{135}$, L. Luminari ${ }^{73 a}$, B. Lund-Jensen ${ }^{153}$, M.S. Lutz ${ }^{160}$, D. Lynn ${ }^{29}$, H. Lyons ${ }^{91}$, R. Lysak ${ }^{140}$, E. Lytken ${ }^{97}$, F. Lyu ${ }^{15 a}$, V. Lyubushkin ${ }^{80}$, T. Lyubushkina ${ }^{80}$, H. Ma ${ }^{29}$, L.L. Ma ${ }^{60 b}$, Y. Ma ${ }^{95}$, G. Maccarrone ${ }^{51}$, A. Macchiolo ${ }^{115}$, C.M. Macdonald ${ }^{148}$, J.C. Macdonald ${ }^{148}$, J. Machado Miguens ${ }^{136}$, D. Madaffari ${ }^{173}$, R. Madar ${ }^{38}$, W.F. Mader ${ }^{48}$, M. Madugoda Ralalage Don ${ }^{129}$, N. Madysa ${ }^{48}$, J. Maeda ${ }^{83}$, T. Maeno ${ }^{29}$, M. Maerker ${ }^{48}$, V. Magerl 52, N. Magini ${ }^{79}$, J. Magro 67a,67c,s, D.J. Mahon ${ }^{39}$, C. Maidantchik 81b, T. Maier 114, A. Maio 139a,139b,139d, K. Maj ${ }^{84 a}$, O. Majersky ${ }^{28 a}$, S. Majewski ${ }^{131}$, Y. Makida ${ }^{82}$, N. Makovec ${ }^{65}$, B. Malaescu ${ }^{135}$, Pa. Malecki ${ }^{85}$, V.P. Maleev 137, F. Malek ${ }^{58,}$ U. Mallik ${ }^{78}$, D. Malon ${ }^{6}$, C. Malone ${ }^{32}$, S. Maltezos ${ }^{10}$, S. Malyukov ${ }^{80}$, J. Mamuzic ${ }^{173}$, G. Mancini ${ }^{51}$, I. Mandić ${ }^{92}$, L. Manhaes de Andrade Filho ${ }^{81 a}$, I.M. Maniatis ${ }^{161}$, J. Manjarres Ramos ${ }^{48}$, K.H. Mankinen ${ }^{97}$, A. Mann ${ }^{114}$, A. Manousos ${ }^{77}$, B. Mansoulie ${ }^{144}$, I. Manthos ${ }^{161}$, S. Manzoni ${ }^{120}$, A. Marantis ${ }^{161}$, G. Marceca ${ }^{30}$, L. Marchese ${ }^{134}$, G. Marchiori 135, M. Marcisovsky ${ }^{140}$, L. Marcoccia ${ }^{74 a, 74 b}$, C. Marcon 97, C.A. Marin Tobon ${ }^{36}$,

M. Marjanovic ${ }^{128}$, Z. Marshall ${ }^{18}$, M.U.F. Martensson ${ }^{171}$, S. Marti-Garcia ${ }^{173}$, C.B. Martin 127, T.A. Martin 177, V.J. Martin 50, B. Martin dit Latour 17, L. Martinelli 75a,75b,

M. Martinez 14,y, P. Martinez Agullo ${ }^{173}$, V.I. Martinez Outschoorn ${ }^{103}$, S. Martin-Haugh ${ }^{143}$, V.S. Martoiu 27b, A.C. Martyniuk 95, A. Marzin 36, S.R. Maschek ${ }^{115}$, L. Masetti ${ }^{100,}$ T. Mashimo ${ }^{162}$, R. Mashinistov ${ }^{111}$, J. Masik ${ }^{101}$, A.L. Maslennikov 122b,122a, L. Massa ${ }^{23 b, 23 a}$, P. Massarotti 70a,70b, P. Mastrandrea 72a,72b, A. Mastroberardino 41b,41a, T. Masubuchi ${ }^{162}$, D. Matakias 29, A. Matic 114, N. Matsuzawa ${ }^{162}$, P. Mättig 24, J. Maurer 27b, B. Maček ${ }^{92}$, D.A. Maximov ${ }^{122 b, 122 a}$, R. Mazini ${ }^{157}$, I. Maznas ${ }^{161}$, S.M. Mazza ${ }^{145}$, J.P. Mc Gowan ${ }^{104}$, S.P. Mc Kee ${ }^{106}$, T.G. McCarthy ${ }^{115}$, W.P. McCormack ${ }^{18}$, E.F. McDonald ${ }^{105}$, J.A. Mcfayden 36, G. Mchedlidze ${ }^{158 b}$, M.A. McKay ${ }^{42}$, K.D. McLean ${ }^{175}$, S.J. McMahon ${ }^{143}$, P.C. McNamara ${ }^{105}$, C.J. McNicol ${ }^{177}$, R.A. McPherson 175, ad, J.E. Mdhluli 33e, Z.A. Meadows 103, S. Meehan 36, T. Megy ${ }^{38}$, S. Mehlhase ${ }^{114}$, A. Mehta 91, B. Meirose ${ }^{43}$, D. Melini ${ }^{159}$, B.R. Mellado Garcia ${ }^{33 e}$, J.D. Mellenthin ${ }^{53}$, M. Melo ${ }^{28 a}$, F. Meloni ${ }^{46}$, A. Melzer ${ }^{24}$, S.B. Menary ${ }^{101}$, E.D. Mendes Gouveia ${ }^{139 a, 139 e}$, L. Meng ${ }^{36}$, X.T. Meng ${ }^{106}$, S. Menke ${ }^{115}$, E. Meoni 41b,41a, S. Mergelmeyer 19, S.A.M. Merkt ${ }^{138}$, C. Merlassino ${ }^{134}$, P. Mermod ${ }^{54}$, L. Merola ${ }^{70 a, 70 b}$, C. Meroni ${ }^{69 a}$, G. Merz ${ }^{106}$, O. Meshkov ${ }^{113,111}$, J.K.R. Meshreki ${ }^{150}$, A. Messina ${ }^{73 a, 73 b}$, J. Metcalfe ${ }^{6}$, A.S. Mete ${ }^{6}$, C. Meyer ${ }^{66}$, J-P. Meyer ${ }^{144}$, H. Meyer Zu Theenhausen ${ }^{61 a}$, F. Miano ${ }^{155}$, M. Michetti ${ }^{19}$, R.P. Middleton ${ }^{143}$, L. Mijović ${ }^{50}$, G. Mikenberg ${ }^{179}$, M. Mikestikova ${ }^{140}$, M. Mikuž ${ }^{92}$, H. Mildner ${ }^{148}$, M. Milesi ${ }^{105}$, A. Milic ${ }^{166}$, C.D. Milke ${ }^{42}$, D.W. Miller ${ }^{37}$, A. Milov ${ }^{179}$, D.A. Milstead 45a,45b, R.A. Mina 152, A.A. Minaenko ${ }^{123}$, M. Miñano Moya ${ }^{173}$, I.A. Minashvili ${ }^{158 b}$, A.I. Mincer ${ }^{125}$, B. Mindur 84a, M. Mineev ${ }^{80}$, Y. Minegishi ${ }^{162}$, L.M. Mir ${ }^{14}$, M. Mironova ${ }^{134}$, A. Mirto 68a,68b, K.P. Mistry ${ }^{136}$, T. Mitani ${ }^{178}$, J. Mitrevski ${ }^{114}$, V.A. Mitsou ${ }^{173}$, M. Mittal 60c , O. Miu ${ }^{166}$, A. Miucci ${ }^{20}$, P.S. Miyagawa ${ }^{148}$, A. Mizukami ${ }^{82}$, J.U. Mjörnmark ${ }^{97}$, T. Mkrtchyan ${ }^{61 a}$, M. Mlynarikova ${ }^{142}$, T. Moa ${ }^{45 a, 45 b}$, S. Mobius ${ }^{53}$, K. Mochizuki ${ }^{110}$, P. Mogg ${ }^{114}$, S. Mohapatra ${ }^{39}$, R. Moles-Valls ${ }^{24}$, M.C. Mondragon ${ }^{107}$, K. Mönig ${ }^{46}$, E. Monnier ${ }^{102}$, A. Montalbano ${ }^{151}$, J. Montejo Berlingen ${ }^{36}$, M. Montella ${ }^{95}$, F. Monticelli ${ }^{89}$, S. Monzani 69a, N. Morange ${ }^{65}$, D. Moreno ${ }^{22 a}$, M. Moreno Llácer ${ }^{173}$, C. Moreno Martinez ${ }^{14}$, P. Morettini ${ }^{55 b}$, 
M. Morgenstern ${ }^{159}$, S. Morgenstern ${ }^{48}$, D. Mori ${ }^{151}$, M. Morii ${ }^{59}$, M. Morinaga ${ }^{178}$, V. Morisbak ${ }^{133}$, A.K. Morley ${ }^{36}$, G. Mornacchi ${ }^{36}$, A.P. Morris ${ }^{95}$, L. Morvaj ${ }^{154}$, P. Moschovakos ${ }^{36}$, B. Moser ${ }^{120}$, M. Mosidze ${ }^{158 b}$, T. Moskalets ${ }^{144}$, H.J. Moss ${ }^{148}$, J. Moss 31,n, E.J.W. Moyse ${ }^{103}$, S. Muanza ${ }^{102}$, J. Mueller ${ }^{138}$, R.S.P. Mueller ${ }^{114}$, D. Muenstermann ${ }^{90}$, G.A. Mullier ${ }^{97}$, D.P. Mungo ${ }^{69 a, 69 b}$, J.L. Munoz Martinez ${ }^{14}$, F.J. Munoz Sanchez ${ }^{101}$, P. Murin ${ }^{28 b}$, W.J. Murray ${ }^{177,143}$, A. Murrone ${ }^{69 a, 69 b}$, M. Muškinja ${ }^{18}$, C. Mwewa ${ }^{33 a}$, A.G. Myagkov ${ }^{123, a i}$, A.A. Myers ${ }^{138}$, J. Myers ${ }^{131}$, M. Myska ${ }^{141}$, B.P. Nachman ${ }^{18}$, O. Nackenhorst ${ }^{47}$, A.Nag Nag ${ }^{48}$, K. Nagai ${ }^{134}$, K. Nagano ${ }^{82}$, Y. Nagasaka ${ }^{62}$, J.L. Nagle ${ }^{29}$, E. Nagy ${ }^{102}$, A.M. Nairz ${ }^{36}$, Y. Nakahama ${ }^{117}$, K. Nakamura ${ }^{82}$, T. Nakamura ${ }^{162}$, H. Nanjo ${ }^{132}$, F. Napolitano ${ }^{61 a}$, R.F. Naranjo Garcia ${ }^{46}$, R. Narayan ${ }^{42}$, I. Naryshkin ${ }^{137}$, T. Naumann ${ }^{46}$, G. Navarro ${ }^{22 a}$, P.Y. Nechaeva ${ }^{111}$, F. Nechansky ${ }^{46}$, T.J. Neep ${ }^{21}$, A. Negri ${ }^{71 a, 71 b}$, M. Negrini ${ }^{23 b}$, C. Nellist ${ }^{119}$, M.E. Nelson ${ }^{45 a, 45 b}$, S. Nemecek ${ }^{140}$, M. Nessi ${ }^{36, e}$, M.S. Neubauer ${ }^{172}$, F. Neuhaus ${ }^{100}$, M. Neumann ${ }^{181}$, R. Newhouse ${ }^{174}$, P.R. Newman ${ }^{21}$, C.W. $\mathrm{Ng}^{138}$, Y.S. $\mathrm{Ng}^{19}$, Y.W.Y. $\mathrm{Ng}^{170}{ }^{17}$, B. Ngair ${ }^{35 e}$, H.D.N. Nguyen ${ }^{102}$, T. Nguyen Manh ${ }^{110}$, E. Nibigira ${ }^{38}$, R.B. Nickerson ${ }^{134}$, R. Nicolaidou ${ }^{144}$, D.S. Nielsen ${ }^{40}$, J. Nielsen ${ }^{145}$, N. Nikiforou ${ }^{11}$, V. Nikolaenko ${ }^{123, \text { ai }}$, I. Nikolic-Audit ${ }^{135}$, K. Nikolopoulos ${ }^{21}$, P. Nilsson ${ }^{29}$, H.R. Nindhito ${ }^{54}$, Y. Ninomiya ${ }^{82}$, A. Nisati ${ }^{73 a}$, N. Nishu ${ }^{60 c}$, R. Nisius ${ }^{115}$, I. Nitsche ${ }^{47}$, T. Nitta ${ }^{178}$, T. Nobe ${ }^{162}$, D.L. Noel ${ }^{32}$, Y. Noguchi ${ }^{86}$, I. Nomidis ${ }^{135}$, M.A. Nomura ${ }^{29}$, M. Nordberg ${ }^{36}$, J. Novak ${ }^{92}$, T. Novak ${ }^{92}$, O. Novgorodova ${ }^{48}$, R. Novotny ${ }^{141}$, L. Nozka ${ }^{130}$, K. Ntekas ${ }^{170}$, E. Nurse ${ }^{95}$, F.G. Oakham ${ }^{34, \text { an }}$, H. Oberlack ${ }^{115}$, J. Ocariz ${ }^{135}$, A. Ochi ${ }^{83}$, I. Ochoa ${ }^{39}$, J.P. Ochoa-Ricoux ${ }^{146 a}$, K. O'Connor ${ }^{26}$, S. Oda ${ }^{88}$, S. Odaka ${ }^{82}$, S. Oerdek ${ }^{53}$, A. Ogrodnik ${ }^{84 a}$, A. Oh ${ }^{101}$, S.H. Oh ${ }^{49}$, C.C. $\mathrm{Ohm}^{153}{ }^{15}$, H. Oide ${ }^{164}$, M.L. Ojeda ${ }^{166}$, H. Okawa ${ }^{168}$, Y. Okazaki ${ }^{86}$, M.W. O'Keefe ${ }^{91}$, Y. Okumura ${ }^{162}$, T. Okuyama ${ }^{82}$, A. Olariu 27b, L.F. Oleiro Seabra ${ }^{139 a}$, S.A. Olivares Pino ${ }^{146 a}$, D. Oliveira Damazio ${ }^{29}$, J.L. Oliver ${ }^{1}$, M.J.R. Olsson ${ }^{170}$, A. Olszewski ${ }^{85}$, J. Olszowska ${ }^{85}$, D.C. O'Neil ${ }^{151}$, A.P. O'neill ${ }^{134}$, A. Onofre ${ }^{139 a, 139 e}$, P.U.E. Onyisi ${ }^{11}$, H. Oppen ${ }^{133}$, R.G. Oreamuno Madriz ${ }^{121}$, M.J. Oreglia ${ }^{37}$, G.E. Orellana ${ }^{89}$, D. Orestano ${ }^{75 a, 75 b}$, N. Orlando ${ }^{14}$, R.S. Orr ${ }^{166}$, V. O'Shea ${ }^{57}$, R. Ospanov ${ }^{60 a}$, G. Otero y Garzon ${ }^{30}$, H. Otono ${ }^{88}$, P.S. Ott ${ }^{61 a}$, G.J. Ottino ${ }^{18}$, M. Ouchrif ${ }^{35 d}$, J. Ouellette ${ }^{29}$, F. Ould-Saada ${ }^{133}$, A. Ouraou ${ }^{144}$, Q. Ouyang ${ }^{15 a}$, M. Owen ${ }^{57}$,

R.E. Owen ${ }^{21}$, V.E. Ozcan ${ }^{12 c}$, N. Ozturk ${ }^{8}$, J. Pacalt ${ }^{130}$, H.A. Pacey ${ }^{32}$, K. Pachal ${ }^{49}$, A. Pacheco Pages ${ }^{14}$, C. Padilla Aranda ${ }^{14}$, S. Pagan Griso ${ }^{18}$, M. Paganini ${ }^{182}$, G. Palacino ${ }^{66}$, S. Palazzo ${ }^{50}$, S. Palestini ${ }^{36}$, M. Palka ${ }^{84 b}$, D. Pallin ${ }^{38}$, P. Palni ${ }^{84 a}$, I. Panagoulias ${ }^{10}$, C.E. Pandini ${ }^{36}$, J.G. Panduro Vazquez ${ }^{94}$, P. Pani ${ }^{46}$, G. Panizzo ${ }^{67 a, 67 c}$, L. Paolozzi ${ }^{54}$, C. Papadatos ${ }^{110}$, K. Papageorgiou ${ }^{9, h}$, S. Parajuli ${ }^{42}$, A. Paramonov ${ }^{6}$, C. Paraskevopoulos ${ }^{10}$, D. Paredes Hernandez ${ }^{63 b}$, S.R. Paredes Saenz ${ }^{134}$, B. Parida ${ }^{179}$, T.H. Park ${ }^{166}$, A.J. Parker ${ }^{31}$, M.A. Parker ${ }^{32}$, F. Parodi 55 b,55a, E.W. Parrish ${ }^{121}$, J.A. Parsons ${ }^{39}$, U. Parzefall ${ }^{52}$, L. Pascual Dominguez ${ }^{135}$, V.R. Pascuzzi ${ }^{18}$, J.M.P. Pasner ${ }^{145}$, F. Pasquali ${ }^{120}$, E. Pasqualucci 73a, S. Passaggio ${ }^{55 b}$, F. Pastore ${ }^{94}$, P. Pasuwan ${ }^{45 a, 45 b}$, S. Pataraia ${ }^{100}$, J.R. Pater ${ }^{101}$,

A. Pathak ${ }^{180, j}$, J. Patton ${ }^{91}$, T. Pauly ${ }^{36}$, J. Pearkes ${ }^{152}$, B. Pearson ${ }^{115}$, M. Pedersen ${ }^{133}$, L. Pedraza Diaz ${ }^{119}$, R. Pedro ${ }^{139 a}$, T. Peiffer ${ }^{53}$, S.V. Peleganchuk ${ }^{122 b, 122 a}$, O. Penc ${ }^{140}$, H. Peng ${ }^{60 a}$, B.S. Peralva ${ }^{81 a}$, M.M. Perego ${ }^{65}$, A.P. Pereira Peixoto ${ }^{139 a}$, L. Pereira Sanchez 45a,45b , D.V. Perepelitsa ${ }^{29}$, F. Peri ${ }^{19}$, L. Perini ${ }^{69 a, 69 b}$, H. Pernegger ${ }^{36}$, S. Perrella ${ }^{139 a}$, A. Perrevoort ${ }^{120}$, K. Peters ${ }^{46}$, R.F.Y. Peters ${ }^{101}$, B.A. Petersen ${ }^{36}$, T.C. Petersen ${ }^{40}$, E. Petit ${ }^{102}$, A. Petridis ${ }^{1}$, C. Petridou ${ }^{161}$, P. Petroff ${ }^{65}$, F. Petrucci ${ }^{75 a, 75 b}$, M. Pettee ${ }^{182}$, N.E. Pettersson ${ }^{103}$, K. Petukhova ${ }^{142}$, A. Peyaud ${ }^{144}$, R. Pezoa ${ }^{146 d}$, L. Pezzotti ${ }^{71 a, 71 b}$, T. Pham ${ }^{105}$, F.H. Phillips ${ }^{107}$, P.W. Phillips ${ }^{143}$, M.W. Phipps ${ }^{172}$, G. Piacquadio ${ }^{154}$, E. Pianori ${ }^{18}$, A. Picazio ${ }^{103}$, R.H. Pickles ${ }^{101}$, R. Piegaia ${ }^{30}$, D. Pietreanu ${ }^{27 b}$, J.E. Pilcher ${ }^{37}$, A.D. Pilkington ${ }^{101}$, M. Pinamonti ${ }^{67 a, 67 c}$, J.L. Pinfold ${ }^{3}$, C. Pitman Donaldson ${ }^{95}$, M. Pitt 
${ }^{160}$, L. Pizzimento ${ }^{74 a, 74 b}$, M.-A. Pleier ${ }^{29}$, V. Pleskot ${ }^{142}$, E. Plotnikova ${ }^{80}$, P. Podberezko 122b,122a, R. Poettgen 97, R. Poggi 54, L. Poggioli 135, I. Pogrebnyak 107, D. Pohl ${ }^{24}$,

I. Pokharel ${ }^{53}$, G. Polesello ${ }^{71 a}$, A. Poley ${ }^{18}$, A. Policicchio ${ }^{73 a, 73 b}$, R. Polifka ${ }^{142}$, A. Polini ${ }^{23 b}$, C.S. Pollard ${ }^{46}$, V. Polychronakos ${ }^{29}$, D. Ponomarenko ${ }^{112}$, L. Pontecorvo ${ }^{36}$, S. Popa ${ }^{27 a}$, G.A. Popeneciu ${ }^{27 d}$, L. Portales ${ }^{5}$, D.M. Portillo Quintero ${ }^{58}$, S. Pospisil ${ }^{141}$, K. Potamianos ${ }^{46}$, I.N. Potrap ${ }^{80}$, C.J. Potter ${ }^{32}$, H. Potti ${ }^{11}$, T. Poulsen ${ }^{97}$, J. Poveda ${ }^{173}$, T.D. Powell ${ }^{148}$, G. Pownall ${ }^{46}$, M.E. Pozo Astigarraga ${ }^{36}$, P. Pralavorio ${ }^{102}$, S. Prell ${ }^{79}$, D. Price ${ }^{101}$, M. Primavera ${ }^{68 a}$, M.L. Proffitt ${ }^{147}$, N. Proklova ${ }^{112}$, K. Prokofiev ${ }^{63 c}$, F. Prokoshin ${ }^{80}$, S. Protopopescu 29, J. Proudfoot 6, M. Przybycien 84a, D. Pudzha ${ }^{137}$, A. Puri 172, P. Puzo ${ }^{65}$, J. Qian ${ }^{106}$, Y. Qin ${ }^{101}$, A. Quadt ${ }^{53}$, M. Queitsch-Maitland ${ }^{36}$, A. Qureshi ${ }^{1}$, M. Racko 28a, F. Ragusa 69a,69b, G. Rahal ${ }^{98}$, J.A. Raine ${ }^{54}$, S. Rajagopalan ${ }^{29}$, A. Ramirez Morales ${ }^{93}$, K. Ran 15a,15d, T. Rashid ${ }^{65}$, D.M. Rauch ${ }^{46}$, F. Rauscher ${ }^{114}$, S. Rave ${ }^{100}$, B. Ravina ${ }^{148}$, I. Ravinovich ${ }^{179}$, J.H. Rawling ${ }^{101}$, M. Raymond ${ }^{36}$, A.L. Read ${ }^{133}$, N.P. Readioff ${ }^{58}$, M. Reale 68a,68b, D.M. Rebuzzi 71a,71b, G. Redlinger ${ }^{29}$, K. Reeves ${ }^{43}$, L. Rehnisch ${ }^{19}$, J. Reichert ${ }^{136}$, D. Reikher ${ }^{160}$, A. Reiss ${ }^{100}$, A. Rej ${ }^{150}$, C. Rembser ${ }^{36}$, A. Renardi ${ }^{46}$, M. Renda ${ }^{27 b}$, M. Rescigno ${ }^{73 a}$, S. Resconi ${ }^{69 a}$, E.D. Resseguie ${ }^{18}$, S. Rettie ${ }^{95}$, B. Reynolds ${ }^{127}$, E. Reynolds 21, O.L. Rezanova 122b,122a, P. Reznicek ${ }^{142}$, E. Ricci 76a,76b, R. Richter ${ }^{115}$, S. Richter ${ }^{46}$, E. Richter-Was ${ }^{84 b}$, O. Ricken ${ }^{24}$, M. Ridel ${ }^{135}$, P. Rieck ${ }^{115}$, O. Rifki ${ }^{46}$, M. Rijssenbeek ${ }^{154}$, A. Rimoldi ${ }^{71 a, 71 b}$, M. Rimoldi ${ }^{46}$, L. Rinaldi ${ }^{23 b}$, G. Ripellino ${ }^{153}$, I. Riu ${ }^{14}$, P. Rivadeneira 46, J.C. Rivera Vergara ${ }^{175}$, F. Rizatdinova ${ }^{129}$, E. Rizvi ${ }^{93}$, C. Rizzi ${ }^{36}$, R.T. Roberts ${ }^{101}$, S.H. Robertson 104,ad, M. Robin ${ }^{46}$, D. Robinson ${ }^{32}$, C.M. Robles Gajardo ${ }^{146 \mathrm{~d}}$,

M. Robles Manzano ${ }^{100}$, A. Robson ${ }^{57}$, A. Rocchi ${ }^{74 a, 74 b}$, E. Rocco ${ }^{100}$, C. Roda ${ }^{72 a, 72 b}$, S. Rodriguez Bosca ${ }^{173}$, D. Rodriguez Rodriguez ${ }^{173}$, A.M. Rodríguez Vera ${ }^{167 b}$, S. Roe ${ }^{36}$, O. Røhne ${ }^{133}$, R. Röhrig ${ }^{115}$, R.A. Rojas ${ }^{146 \mathrm{~d}}$, B. Roland ${ }^{52}$, C.P.A. Roland ${ }^{66}$, J. Roloff ${ }^{29}$, A. Romaniouk ${ }^{112}$, M. Romano ${ }^{23 b, 23 a}$, N. Rompotis ${ }^{91}$, M. Ronzani ${ }^{125}$, L. Roos ${ }^{135}$, S. Rosati 73a, G. Rosin 103, B.J. Rosser ${ }^{136}$, E. Rossi ${ }^{46}$, E. Rossi ${ }^{75 a}, 75 b$, E. Rossi 70a,70b, L.P. Rossi 55b, L. Rossini 69a,69b, R. Rosten 14, M. Rotaru 27b, B. Rottler 52, D. Rousseau 65, G. Rovelli 71a,71b, A. Roy ${ }^{11}$, D. Roy ${ }^{33 e}$, A. Rozanov ${ }^{102}$, Y. Rozen ${ }^{159}$, X. Ruan ${ }^{33 e}$, F. Rühr ${ }^{52}$,

A. Ruiz-Martinez ${ }^{173}$, A. Rummler ${ }^{36}$, Z. Rurikova ${ }^{52}$, N.A. Rusakovich ${ }^{80}$, H.L. Russell ${ }^{104}$, L. Rustige ${ }^{38,47}$, J.P. Rutherfoord ${ }^{7}$, E.M. Rüttinger ${ }^{148}$, M. Rybar ${ }^{39}$, G. Rybkin ${ }^{65}$, E.B. Rye 133, A. Ryzhov ${ }^{123}$, J.A. Sabater Iglesias ${ }^{46}$, P. Sabatini ${ }^{53}$, S. Sacerdoti ${ }^{65}$, H.F-W. Sadrozinski 145, R. Sadykov ${ }^{80}$, F. Safai Tehrani 73a, B. Safarzadeh Samani 155, M. Safdari 152, P. Saha ${ }^{121}$, S. Saha ${ }^{104}$, M. Sahinsoy ${ }^{61 a}$, A. Sahu ${ }^{181}$, M. Saimpert ${ }^{36}$, M. Saito ${ }^{162}$, T. Saito ${ }^{162}$, H. Sakamoto ${ }^{162}$, D. Salamani ${ }^{54}$, G. Salamanna ${ }^{75 a, 75 b}$, J.E. Salazar Loyola 146d, A. Salnikov 152, J. Salt ${ }^{173}$, A. Salvador Salas ${ }^{14}$, D. Salvatore ${ }^{41 b, 41 a}$, F. Salvatore ${ }^{155}$, A. Salvucci 63a,63b,63c, A. Salzburger 36, J. Samarati ${ }^{36}$, D. Sammel 52, D. Sampsonidis ${ }^{161}$, D. Sampsonidou ${ }^{161}$, J. Sánchez ${ }^{173}$, A. Sanchez Pineda ${ }^{67 a, 36,67 c}$, H. Sandaker ${ }^{133}$, C.O. Sander ${ }^{46}$, I.G. Sanderswood ${ }^{90}$, M. Sandhoff ${ }^{181}$, C. Sandoval ${ }^{22 a}$, D.P.C. Sankey ${ }^{143}$, M. Sannino 55b,55a, Y. Sano 117, A. Sansoni 51, C. Santoni ${ }^{38}$, H. Santos 139a,139b, S.N. Santpur ${ }^{18}$, A. Santra ${ }^{173}$, A. Sapronov ${ }^{80}$, J.G. Saraiva ${ }^{139 a, 139 d}$, O. Sasaki ${ }^{82}$, K. Sato ${ }^{168}$, F. Sauerburger 52, E. Sauvan 5, P. Savard 166,an, R. Sawada ${ }^{162}$, C. Sawyer 143, L. Sawyer 96,ah, I. Sayago Galvan ${ }^{173}$, C. Sbarra ${ }^{23 b}$, A. Sbrizzi ${ }^{23 a}$, T. Scanlon ${ }^{95}$, J. Schaarschmidt ${ }^{147}$, P. Schacht ${ }^{115}$, B.M. Schachtner ${ }^{114}$, D. Schaefer ${ }^{37}$, L. Schaefer ${ }^{136}$, J. Schaeffer ${ }^{100}$, S. Schaepe 36, U. Schäfer ${ }^{100}$, A.C. Schaffer ${ }^{65}$, D. Schaile ${ }^{114}$, R.D. Schamberger 154, E. Schanet ${ }^{114}$, N. Scharmberg ${ }^{101}$, V.A. Schegelsky ${ }^{137}$, D. Scheirich ${ }^{142}$, F. Schenck ${ }^{19}$, M. Schernau ${ }^{170}$, C. Schiavi 55b,55a, L.K. Schildgen ${ }^{24}$, Z.M. Schillaci ${ }^{26}$, E.J. Schioppa ${ }^{68 a, 68 b}$, M. Schioppa 41b,41a, K.E. Schleicher ${ }^{52}$, S. Schlenker ${ }^{36}$, K.R. Schmidt-Sommerfeld ${ }^{115}$, K. Schmieden ${ }^{36}$, C. Schmitt ${ }^{100}$, S. Schmitt ${ }^{46}$, J.C. Schmoeckel ${ }^{46}$, L. Schoeffel ${ }^{144}$, A. Schoening ${ }^{61 b}$, 
P.G. Scholer ${ }^{52}$, E. Schopf ${ }^{134}$, M. Schott ${ }^{100}$, J.F.P. Schouwenberg ${ }^{119}$, J. Schovancova ${ }^{36}$, S. Schramm ${ }^{54}$, F. Schroeder ${ }^{181}$, A. Schulte ${ }^{100}$, H-C. Schultz-Coulon ${ }^{61 a}$, M. Schumacher ${ }^{52}$, B.A. Schumm 145, Ph. Schune ${ }^{144}$, A. Schwartzman 152, T.A. Schwarz 106, Ph. Schwemling ${ }^{144}$, R. Schwienhorst ${ }^{107}$, A. Sciandra ${ }^{145}$, G. Sciolla ${ }^{26}$, M. Scodeggio ${ }^{46}$, M. Scornajenghi ${ }^{41 b, 41 a}$, F. Scuri ${ }^{72 a}$, F. Scutti ${ }^{105}$, L.M. Scyboz ${ }^{115}$, C.D. Sebastiani ${ }^{73 a}, 73 b$, P. Seema ${ }^{19}$, S.C. Seidel 118 , A. Seiden ${ }^{145}$, B.D. Seidlitz ${ }^{29}$, T. Seiss ${ }^{37}$, C. Seitz ${ }^{46}$, J.M. Seixas ${ }^{81 b}$, G. Sekhniaidze 70a, S.J. Sekula ${ }^{42}$, N. Semprini-Cesari ${ }^{23 b, 23 a}$, S. Sen ${ }^{49}$, C. Serfon ${ }^{29}$, L. Serin ${ }^{65}$, L. Serkin 67a,67b, M. Sessa ${ }^{60 a}$, H. Severini ${ }^{128}$, S. Sevova ${ }^{152}$, F. Sforza ${ }^{55 b, 55 a}$, A. Sfyrla ${ }^{54}$,

E. Shabalina ${ }^{53}$, J.D. Shahinian ${ }^{145}$, N.W. Shaikh 45a,45b, D. Shaked Renous ${ }^{179}$, L.Y. Shan ${ }^{15 a}$, M. Shapiro ${ }^{18}$, A. Sharma ${ }^{134}$, A.S. Sharma 1, P.B. Shatalov ${ }^{124}$, K. Shaw ${ }^{155}$, S.M. Shaw ${ }^{101}$, M. Shehade ${ }^{179}$, Y. Shen ${ }^{128}$, A.D. Sherman ${ }^{25}$, P. Sherwood ${ }^{95}$, L. Shi ${ }^{157}$, S. Shimizu ${ }^{82}$, C.O. Shimmin ${ }^{182}$, Y. Shimogama ${ }^{178}$, M. Shimojima ${ }^{116}$, I.P.J. Shipsey ${ }^{134}$, S. Shirabe ${ }^{164}$, M. Shiyakova ${ }^{80, a b}$, J. Shlomi ${ }^{179}$, A. Shmeleva ${ }^{111}$, M.J. Shochet ${ }^{37}$, J. Shojaii ${ }^{105}$, D.R. Shope ${ }^{128}$, S. Shrestha ${ }^{127}$, E.M. Shrif ${ }^{33 e}$, E. Shulga ${ }^{179}$, P. Sicho ${ }^{140}$, A.M. Sickles ${ }^{172}$, E. Sideras Haddad ${ }^{33 e}$, O. Sidiropoulou ${ }^{36}$, A. Sidoti ${ }^{23 b, 23 a}$, F. Siegert ${ }^{48}$, Dj. Sijacki ${ }^{16}$, M.Jr. Silva ${ }^{180}$, M.V. Silva Oliveira ${ }^{81 a}$, S.B. Silverstein ${ }^{45 a}$, S. Simion ${ }^{65}$, R. Simoniello ${ }^{100}$, C.J. Simpson-allsop ${ }^{21}$, S. Simsek ${ }^{12 b}$, P. Sinervo ${ }^{166}$, V. Sinetckii ${ }^{113}$, S. Singh ${ }^{151}$, M. Sioli 23b,23a, I. Siral 131, S.Yu. Sivoklokov ${ }^{113}$, J. Sjölin 45a,45b, A. Skaf 53, E. Skorda 97, P. Skubic ${ }^{128}$, M. Slawinska ${ }^{85}$, K. Sliwa ${ }^{169}$, R. Slovak ${ }^{142}$, V. Smakhtin ${ }^{179}$, B.H. Smart ${ }^{143}$, J. Smiesko 28b, N. Smirnov ${ }^{112}$, S.Yu. Smirnov ${ }^{112}$, Y. Smirnov ${ }^{112}$, L.N. Smirnova 113,t, O. Smirnova ${ }^{97}$, J.W. Smith ${ }^{53}$, M. Smizanska ${ }^{90}$, K. Smolek ${ }^{141}$, A. Smykiewicz ${ }^{85}$, A.A. Snesarev ${ }^{111}$, H.L. Snoek ${ }^{120}$, I.M. Snyder ${ }^{131}$, S. Snyder ${ }^{29}$, R. Sobie ${ }^{175, \text { ad }}$, A. Soffer ${ }^{160}$, A. Søgaard ${ }^{50}$, F. Sohns ${ }^{53}$, C.A. Solans Sanchez ${ }^{36}$, E.Yu. Soldatov ${ }^{112}$, U. Soldevila ${ }^{173}$, A.A. Solodkov ${ }^{123}$, A. Soloshenko ${ }^{80}$, O.V. Solovyanov ${ }^{123}$, V. Solovyev 137, P. Sommer ${ }^{148}$, H. Son ${ }^{169}$, W. Song ${ }^{143}$, W.Y. Song ${ }^{167 b}$, A. Sopczak ${ }^{141}$, A.L. Sopio ${ }^{95}$, F. Sopkova ${ }^{28 b}$, C.L. Sotiropoulou ${ }^{72 a, 72 b}$, S. Sottocornola 71a,71b, R. Soualah 67a,67c,g, A.M. Soukharev 122b,122a, D. South 46, S. Spagnolo 68a,68b, M. Spalla ${ }^{115}$, M. Spangenberg 177, F. Spanò ${ }^{94}$, D. Sperlich 52, T.M. Spieker ${ }^{61 a}$, G. Spigo ${ }^{36}$, M. Spina ${ }^{155}$, D.P. Spiteri ${ }^{57}$, M. Spousta ${ }^{142}$, A. Stabile 69a,69b, B.L. Stamas ${ }^{121}$, R. Stamen ${ }^{61 a}$, M. Stamenkovic ${ }^{120}$, E. Stanecka ${ }^{85}$, B. Stanislaus ${ }^{134}$, M.M. Stanitzki ${ }^{46}$, M. Stankaityte ${ }^{134}$, B. Stapf ${ }^{120}$, E.A. Starchenko ${ }^{123}$, G.H. Stark ${ }^{145}$, J. Stark ${ }^{58}$, P. Staroba ${ }^{140}$, P. Starovoitov ${ }^{61 a}$, S. Stärz ${ }^{104}$, R. Staszewski ${ }^{85}$, G. Stavropoulos ${ }^{44}$, M. Stegler ${ }^{46}$, P. Steinberg 29, A.L. Steinhebel 131, B. Stelzer 151, H.J. Stelzer 138, O. Stelzer-Chilton 167a, H. Stenzel ${ }^{56}$, T.J. Stevenson ${ }^{155}$, G.A. Stewart ${ }^{36}$, M.C. Stockton ${ }^{36}$, G. Stoicea ${ }^{27 b}$, M. Stolarski 139a, S. Stonjek ${ }^{115}$, A. Straessner ${ }^{48}$, J. Strandberg 153, S. Strandberg ${ }^{45 a, 45 b}$, M. Strauss ${ }^{128}$, T. Strebler ${ }^{102}$, P. Strizenec ${ }^{28 b}$, R. Ströhmer ${ }^{176}$, D.M. Strom ${ }^{131}$, R. Stroynowski ${ }^{42}$, A. Strubig ${ }^{50}$, S.A. Stucci ${ }^{29}$, B. Stugu ${ }^{17}$, J. Stupak ${ }^{128}$, N.A. Styles ${ }^{46}$, D. $\mathrm{Su}^{152}$, W. Su ${ }^{60 c, 147}$, S. Suchek ${ }^{61 \mathrm{a}}$, V.V. Sulin ${ }^{111}$, M.J. Sullivan ${ }^{91}$, D.M.S. Sultan ${ }^{54}$, S. Sultansoy ${ }^{4 c}$, T. Sumida ${ }^{86}$, S. Sun ${ }^{106}$, X. Sun ${ }^{101}$, K. Suruliz ${ }^{155}$, C.J.E. Suster ${ }^{156}$, M.R. Sutton ${ }^{155}$, S. Suzuki ${ }^{82}$, M. Svatos ${ }^{140}$, M. Swiatlowski ${ }^{167 a}$, S.P. Swift ${ }^{2}$, T. Swirski ${ }^{176}$, A. Sydorenko ${ }^{100}$, I. Sykora ${ }^{28 a}$, M. Sykora ${ }^{142}$, T. Sykora ${ }^{142}$, D. Ta ${ }^{100}$, K. Tackmann ${ }^{46, z}$, J. Taenzer ${ }^{160}$, A. Taffard ${ }^{170}$, R. Tafirout ${ }^{167 a}$, R. Takashima ${ }^{87}$, K. Takeda ${ }^{83}$, T. Takeshita ${ }^{149}$, E.P. Takeva ${ }^{50}$, Y. Takubo ${ }^{82}$, M. Talby ${ }^{102}$, A.A. Talyshev ${ }^{122 b, 122 a}$, K.C. Tam ${ }^{63 b}$, N.M. Tamir ${ }^{160}$, J. Tanaka ${ }^{162}$, R. Tanaka ${ }^{65}$, S. Tapia Araya ${ }^{172}$, S. Tapprogge ${ }^{100}$, A. Tarek Abouelfadl Mohamed ${ }^{107}$, S. Tarem ${ }^{159}$, K. Tariq ${ }^{60 b}$, G. Tarna 27b,d, G.F. Tartarelli 69a, P. Tas ${ }^{142}$, M. Tasevsky ${ }^{140}$, T. Tashiro ${ }^{86}$, E. Tassi ${ }^{41 b, 41 a}$, A. Tavares Delgado ${ }^{139 a}$, Y. Tayalati ${ }^{35 e}$, A.J. Taylor ${ }^{50}$, G.N. Taylor ${ }^{105}$, W. Taylor ${ }^{167 b}$, H. Teagle ${ }^{91}$, A.S. Tee ${ }^{90}$, R. Teixeira De Lima ${ }^{152}$, P. Teixeira-Dias ${ }^{94}$, H. Ten Kate ${ }^{36}$, J.J. Teoh ${ }^{120}$, S. Terada ${ }^{82}$, K. Terashi ${ }^{162}$, J. Terron ${ }^{99}$, S. Terzo ${ }^{14}$, M. Testa ${ }^{51}$, R.J. Teuscher ${ }^{166, a d}$, S.J. Thais ${ }^{182}$, 
N. Themistokleous ${ }^{50}$, T. Theveneaux-Pelzer ${ }^{46}$, F. Thiele ${ }^{40}$, D.W. Thomas ${ }^{94}$, J.O. Thomas 42 , J.P. Thomas ${ }^{21}$, E.A. Thompson ${ }^{46}$, P.D. Thompson ${ }^{21}$, E. Thomson ${ }^{136}$, E.J. Thorpe ${ }^{93}$, R.E. Ticse Torres ${ }^{53}$, V.O. Tikhomirov ${ }^{111, a j}$, Yu.A. Tikhonov ${ }^{122 b, 122 a}$, S. Timoshenko ${ }^{112}$, P. Tipton ${ }^{182}$, S. Tisserant ${ }^{102}$, K. Todome ${ }^{23 b, 23 a}$, S. Todorova-Nova ${ }^{142}$, S. Todt ${ }^{48}$, J. Tojo ${ }^{88}$, S. Tokár ${ }^{28 a}$, K. Tokushuku ${ }^{82}$, E. Tolley ${ }^{127}$, R. Tombs ${ }^{32}$, K.G. Tomiwa ${ }^{33 e}$, M. Tomoto 117, L. Tompkins ${ }^{152}$, P. Tornambe ${ }^{103}$, E. Torrence ${ }^{131}$, H. Torres ${ }^{48}$, E. Torró Pastor ${ }^{147}$, C. Tosciri ${ }^{134}$, J. Toth ${ }^{102, \text { ac }, \text { D.R. Tovey }}{ }^{148}$, A. Traeet ${ }^{17}$, C.J. Treado ${ }^{125}$, T. Trefzger ${ }^{176}$, F. Tresoldi ${ }^{155}$, A. Tricoli ${ }^{29}$, I.M. Trigger ${ }^{167 a}$, S. Trincaz-Duvoid ${ }^{135}$, D.A. Trischuk ${ }^{174}$, W. Trischuk 166, B. Trocmé ${ }^{58}$, A. Trofymov ${ }^{65}$, C. Troncon 69a, F. Trovato ${ }^{155}$, L. Truong ${ }^{33 c}$, M. Trzebinski ${ }^{85}$, A. Trzupek ${ }^{85}$, F. Tsai ${ }^{46}$, J.C-L. Tseng ${ }^{134}$, P.V. Tsiareshka 108,ag, A. Tsirigotis ${ }^{161, \mathrm{w}}$, V. Tsiskaridze ${ }^{154}$, E.G. Tskhadadze ${ }^{158 \mathrm{a}}$, M. Tsopoulou ${ }^{161}$, I.I. Tsukerman ${ }^{124}$, V. Tsulaia ${ }^{18}$, S. Tsuno ${ }^{82}$, D. Tsybychev ${ }^{154}$, Y. Tu ${ }^{63 b}$, A. Tudorache ${ }^{27 b}$, V. Tudorache ${ }^{27 b}$, T.T. Tulbure ${ }^{27 a}$, A.N. Tuna ${ }^{59}$, S. Turchikhin ${ }^{80}$, D. Turgeman ${ }^{179}$, I. Turk Cakir 4b,u , R.J. Turner ${ }^{21}$, R. Turra ${ }^{69 a}$, P.M. Tuts ${ }^{39}$, S. Tzamarias ${ }^{161}$, E. Tzovara ${ }^{100}$, G. Ucchielli ${ }^{47}$, K. Uchida ${ }^{162}$, F. Ukegawa ${ }^{168}$, G. Unal ${ }^{36}$, A. Undrus ${ }^{29}$, G. Unel ${ }^{170}$, F.C. Ungaro ${ }^{105}$, Y. Unno ${ }^{82}$, K. Uno ${ }^{162}$, J. Urban ${ }^{28 b}$, P. Urquijo ${ }^{105}$, G. Usai ${ }^{8}$, Z. Uysal 12d, V. Vacek ${ }^{141}$, B. Vachon ${ }^{104}$, K.O.H. Vadla ${ }^{133}$, A. Vaidya ${ }^{95}$, C. Valderanis ${ }^{114}$, E. Valdes Santurio ${ }^{45 a, 45 b}$, M. Valente ${ }^{54}$, S. Valentinetti ${ }^{23 b, 23 a}$, A. Valero ${ }^{173}$, L. Valéry ${ }^{46}$, R.A. Vallance ${ }^{21}$, A. Vallier ${ }^{36}$, J.A. Valls Ferrer ${ }^{173}$, T.R. Van Daalen ${ }^{14}$, P. Van Gemmeren ${ }^{6}$, I. Van Vulpen ${ }^{120}$, M. Vanadia ${ }^{74 a, 74 b}$, W. Vandelli ${ }^{36}$, M. Vandenbroucke ${ }^{144}$, E.R. Vandewall 129, A. Vaniachine ${ }^{165}$, D. Vannicola ${ }^{73 a, 73 b}$, R. Vari ${ }^{73 a}$, E.W. Varnes ${ }^{7}$, C. Varni ${ }^{55 b, 55 a}$, T. Varol ${ }^{157}$, D. Varouchas ${ }^{65}$, K.E. Varvell ${ }^{156}$, M.E. Vasile ${ }^{27 b}$, G.A. Vasquez ${ }^{175}$, F. Vazeille 38, D. Vazquez Furelos ${ }^{14}$, T. Vazquez Schroeder ${ }^{36}$, J. Veatch ${ }^{53}$, V. Vecchio ${ }^{101}$, M.J. Veen ${ }^{120}$, L.M. Veloce ${ }^{166}$, F. Veloso 139a,139c, S. Veneziano ${ }^{73 a}$, A. Ventura 68a,68b, N. Venturi ${ }^{36}$, A. Verbytskyi ${ }^{115}$, V. Vercesi ${ }^{71 a}$, M. Verducci ${ }^{72 a, 72 b}$, C.M. Vergel Infante ${ }^{79}$, C. Vergis ${ }^{24}$, W. Verkerke ${ }^{120}$, A.T. Vermeulen ${ }^{120}$, J.C. Vermeulen ${ }^{120}$, C. Vernieri ${ }^{152}$, M.C. Vetterli ${ }^{151 \text {, an }}$, N. Viaux Maira ${ }^{146 d}$, T. Vickey ${ }^{148}$, O.E. Vickey Boeriu ${ }^{148}$, G.H.A. Viehhauser ${ }^{134}$, L. Vigani 61b, M. Villa ${ }^{23 b, 23 a}$, M. Villaplana Perez ${ }^{3}$, E.M. Villhauer ${ }^{50}$, E. Vilucchi ${ }^{51}$, M.G. Vincter ${ }^{34}$, G.S. Virdee ${ }^{21}$, A. Vishwakarma ${ }^{50}$, C. Vittori ${ }^{23 b, 23 a}$, I. Vivarelli ${ }^{155}$, M. Vogel ${ }^{181}$, P. Vokac ${ }^{141}$, S.E. von Buddenbrock ${ }^{33 e}$, E. Von Toerne ${ }^{24}$, V. Vorobel ${ }^{142}$, K. Vorobev ${ }^{112}$, M. Vos ${ }^{173}$, J.H. Vossebeld ${ }^{91}$, M. Vozak ${ }^{101}$, N. Vranjes ${ }^{16}$, M. Vranjes Milosavljevic ${ }^{16}$, V. Vrba ${ }^{141}$, M. Vreeswijk ${ }^{120}$, R. Vuillermet ${ }^{36}$, I. Vukotic ${ }^{37}$, S. Wada ${ }^{168}$, P. Wagner ${ }^{24}$, W. Wagner ${ }^{181}$, J. Wagner-Kuhr ${ }^{114}$, S. Wahdan ${ }^{181}$, H. Wahlberg ${ }^{89}$, R. Wakasa ${ }^{168}$, V.M. Walbrecht ${ }^{115}$, J. Walder ${ }^{90}$, R. Walker ${ }^{114}$, S.D. Walker ${ }^{94}$, W. Walkowiak ${ }^{150}$, V. Wallangen ${ }^{45 a, 45 b}$, A.M. Wang ${ }^{59}$, A.Z. Wang ${ }^{180}$, C. Wang ${ }^{60 c}$, F. Wang ${ }^{180}$, H. Wang ${ }^{18}$, H. Wang ${ }^{3}$, J. Wang 63a, J. Wang 61b, P. Wang ${ }^{42}$, Q. Wang ${ }^{128}$, R.-J. Wang ${ }^{100}$, R. Wang 60a, R. Wang ${ }^{6}$, S.M. Wang ${ }^{157}$, W.T. Wang ${ }^{60 a}$, W. Wang ${ }^{15 c}$, W.X. Wang ${ }^{60 a}$, Y. Wang ${ }^{60 a}$, Z. Wang 60c, C. Wanotayaroj ${ }^{46}$, A. Warburton ${ }^{104}$, C.P. Ward ${ }^{32}$, D.R. Wardrope ${ }^{95}$, N. Warrack ${ }^{57}$, A. Washbrook ${ }^{50}$, A.T. Watson ${ }^{21}$, M.F. Watson ${ }^{21}$, G. Watts ${ }^{147}$, B.M. Waugh ${ }^{95}$, A.F. Webb ${ }^{11}$, C. Weber ${ }^{29}$, M.S. Weber ${ }^{20}$, S.A. Weber ${ }^{34}$, S.M. Weber ${ }^{61 a}$, A.R. Weidberg ${ }^{134}$, J. Weingarten ${ }^{47}$, M. Weirich ${ }^{100}$, C. Weiser ${ }^{52}$, P.S. Wells ${ }^{36}$, T. Wenaus ${ }^{29}$, T. Wengler ${ }^{36}$, S. Wenig ${ }^{36}$, N. Wermes ${ }^{24}$, M.D. Werner ${ }^{79}$, M. Wessels ${ }^{61 a}$, T.D. Weston ${ }^{20}$, K. Whalen ${ }^{131}$, N.L. Whallon ${ }^{147}$, A.M. Wharton ${ }^{90}$, A.S. White ${ }^{106}$, A. White ${ }^{8}$, M.J. White ${ }^{1}$, D. Whiteson ${ }^{170}$, B.W. Whitmore ${ }^{90}$, W. Wiedenmann ${ }^{180}$, C. Wiel ${ }^{48}$, M. Wielers ${ }^{143}$, N. Wieseotte ${ }^{100}$, C. Wiglesworth ${ }^{40}$, L.A.M. Wiik-Fuchs ${ }^{52}$, H.G. Wilkens ${ }^{36}$, L.J. Wilkins ${ }^{94}$, H.H. Williams ${ }^{136}$, S. Williams ${ }^{32}$, C. Willis ${ }^{107}$, S. Willocq ${ }^{103}$, P.J. Windischhofer ${ }^{134}$, I. Wingerter-Seez ${ }^{5}$, E. Winkels ${ }^{155}$, F. Winklmeier ${ }^{131}$, B.T. Winter ${ }^{52}$, M. Wittgen ${ }^{152}$, M. Wobisch ${ }^{96}$, A. Wolf ${ }^{100}$, T.M.H. Wolf ${ }^{120}$, R. Wölker ${ }^{134}$, J. Wollrath ${ }^{52}$, 
M.W. Wolter ${ }^{85}$, H. Wolters ${ }^{139 a, 139 c}$, V.W.S. Wong ${ }^{174}$, N.L. Woods ${ }^{145}$, S.D. Worm ${ }^{46}$, B.K. Wosiek ${ }^{85}$, K.W. Woźniak ${ }^{85}$, K. Wraight ${ }^{57}$, S.L. Wu ${ }^{180}$, X. Wu ${ }^{54}$, Y. Wu ${ }^{60 a}$, J. Wuerzinger ${ }^{134}$, T.R. Wyatt ${ }^{101}$, B.M. Wynne ${ }^{50}$, S. Xella ${ }^{40}$, Z. Xi ${ }^{106}$, L. Xia ${ }^{177}$, J. Xiang ${ }^{63 c}$, X. Xiao ${ }^{106}$, X. Xie ${ }^{60 a}$, I. Xiotidis ${ }^{155}$, D. Xu ${ }^{15 a}, \quad$ H. Xu ${ }^{60 a}$, H. Xu ${ }^{60 a}$, L. Xu ${ }^{29}$, T. Xu ${ }^{144}$, W. Xu ${ }^{106}$, Z. Xu ${ }^{60 b}$, Z. Xu ${ }^{152}$, B. Yabsley ${ }^{156}$, S. Yacoob ${ }^{33 a}$, K. Yajima ${ }^{132}$, D.P. Yallup ${ }^{95}$, N. Yamaguchi ${ }^{88}$, Y. Yamaguchi ${ }^{164}$, A. Yamamoto ${ }^{82}$, M. Yamatani ${ }^{162}$, T. Yamazaki ${ }^{162}$, Y. Yamazaki ${ }^{83}$, J. Yan ${ }^{60 c}$, Z. Yan ${ }^{25}$, H.J. Yang ${ }^{60 c, 60 d, ~ H . T . ~ Y a n g ~}{ }^{18}$, S. Yang ${ }^{60 a}$, T. Yang ${ }^{63 c}$, X. Yang ${ }^{60 b, 58}$, Y. Yang ${ }^{162}$, Z. Yang ${ }^{60 a}$, W-M. Yao ${ }^{18}$, Y.C. Yap ${ }^{46}$, Y. Yasu ${ }^{82}$, E. Yatsenko ${ }^{60 c, 60 d}$, H. Ye ${ }^{15 c}$, J. Ye ${ }^{42}$, S. Ye ${ }^{29}$, I. Yeletskikh ${ }^{80}$, M.R. Yexley ${ }^{90}$, E. Yigitbasi ${ }^{25}$, P. Yin ${ }^{39}$, K. Yorita ${ }^{178}$, K. Yoshihara ${ }^{79}$, C.J.S. Young ${ }^{36}$, C. Young ${ }^{152}$, J. Yu ${ }^{79}$, R. Yuan ${ }^{60 b, i}$, X. Yue ${ }^{61 a}$, M. Zaazoua ${ }^{35 e}$, B. Zabinski ${ }^{85}$, G. Zacharis ${ }^{10}$, E. Zaffaroni ${ }^{54}$, J. Zahreddine ${ }^{135}$, A.M. Zaitsev ${ }^{123, \text { ai }}$, T. Zakareishvili ${ }^{158 b}$, N. Zakharchuk ${ }^{34}$, S. Zambito ${ }^{59}$, D. Zanzi ${ }^{36}$, D.R. Zaripovas ${ }^{57}$, S.V. Zeißner ${ }^{47}$, C. Zeitnitz ${ }^{181}$, G. Zemaityte ${ }^{134}$, J.C. Zeng ${ }^{172}$, O. Zenin ${ }^{123}$, T. Ženišs ${ }^{28 a}$, D. Zerwas ${ }^{65}$, M. Zgubič ${ }^{134}$, B. Zhang ${ }^{15 c}$, D.F. Zhang ${ }^{15 b}$, G. Zhang ${ }^{15 b}$, J. Zhang ${ }^{6}$, Kaili. Zhang ${ }^{15 a}$, L. Zhang ${ }^{15 c}$, L. Zhang ${ }^{60 a}$, M. Zhang ${ }^{172}$, R. Zhang ${ }^{180}$, S. Zhang ${ }^{106}$, X. Zhang ${ }^{60 c}$, X. Zhang ${ }^{60 b}$, Y. Zhang ${ }^{15 a, 15 d}$, Z. Zhang ${ }^{63 a}$, Z. Zhang ${ }^{65}$, P. Zhao ${ }^{49}$, Z. Zhao ${ }^{60 a}$, A. Zhemchugov ${ }^{80}$, Z. Zheng ${ }^{106}$, D. Zhong ${ }^{172}$, B. Zhou ${ }^{106}$, C. Zhou ${ }^{180}$, H. Zhou ${ }^{7}$, M.S. Zhou ${ }^{15 a, 15 d}$, M. Zhou ${ }^{154}$, N. Zhou ${ }^{60 c}$, Y. Zhou ${ }^{7}$, C.G. Zhu ${ }^{60 b}$, C. Zhu ${ }^{15 a, 15 d}$, H.L. Zhu ${ }^{60 a}$, H. Zhu ${ }^{15 a}$, J. Zhu ${ }^{106}$, Y. Zhu ${ }^{60 a}$, X. Zhuang ${ }^{15 a}$, K. Zhukov ${ }^{111}$, V. Zhulanov ${ }^{122 b, 122 a}$, D. Zieminska ${ }^{66}$, N.I. Zimine ${ }^{80}$, S. Zimmermann ${ }^{52}$, Z. Zinonos ${ }^{115}$, M. Ziolkowski ${ }^{150}$, L. Živković ${ }^{16}$, G. Zobernig ${ }^{180}$, A. Zoccoli ${ }^{23 b, 23 a}$, K. Zoch ${ }^{53}$, T.G. Zorbas ${ }^{148}$, R. Zou ${ }^{37}$ and L. Zwalinski ${ }^{36}$

1 Department of Physics, University of Adelaide, Adelaide; Australia

2 Physics Department, SUNY Albany, Albany NY; United States of America

3 Department of Physics, University of Alberta, Edmonton AB; Canada

$4{ }^{(a)}$ Department of Physics, Ankara University, Ankara; ${ }^{(b)}$ Istanbul Aydin University, Application and Research Center for Advanced Studies, Istanbul; ${ }^{(c)}$ Division of Physics, TOBB University of Economics and Technology, Ankara; Turkey

5 LAPP, Université Grenoble Alpes, Université Savoie Mont Blanc, CNRS/IN2P3, Annecy; France

6 High Energy Physics Division, Argonne National Laboratory, Argonne IL; United States of America

7 Department of Physics, University of Arizona, Tucson AZ; United States of America

8 Department of Physics, University of Texas at Arlington, Arlington TX; United States of America

9 Physics Department, National and Kapodistrian University of Athens, Athens; Greece

10 Physics Department, National Technical University of Athens, Zografou; Greece

11 Department of Physics, University of Texas at Austin, Austin TX; United States of America

$12{ }^{(a)}$ Bahcesehir University, Faculty of Engineering and Natural Sciences, Istanbul; ${ }^{(b)}$ Istanbul Bilgi University, Faculty of Engineering and Natural Sciences, Istanbul; ${ }^{(c)}$ Department of Physics, Bogazici University, Istanbul; ${ }^{(d)}$ Department of Physics Engineering, Gaziantep University, Gaziantep; Turkey

13 Institute of Physics, Azerbaijan Academy of Sciences, Baku; Azerbaijan

14 Institut de Física d'Altes Energies (IFAE), Barcelona Institute of Science and Technology, Barcelona; Spain

$15{ }^{(a)}$ Institute of High Energy Physics, Chinese Academy of Sciences, Beijing; ${ }^{(b)}$ Physics Department, Tsinghua University, Beijing; ${ }^{(c)}$ Department of Physics, Nanjing University, Nanjing; ${ }^{(d)}$ University of Chinese Academy of Science (UCAS), Beijing; China

16 Institute of Physics, University of Belgrade, Belgrade; Serbia

17 Department for Physics and Technology, University of Bergen, Bergen; Norway

18 Physics Division, Lawrence Berkeley National Laboratory and University of California, Berkeley CA; United States of America 
19 Institut für Physik, Humboldt Universität zu Berlin, Berlin; Germany

20 Albert Einstein Center for Fundamental Physics and Laboratory for High Energy Physics, University of Bern, Bern; Switzerland

21 School of Physics and Astronomy, University of Birmingham, Birmingham; United Kingdom

$22{ }^{(a)}$ Facultad de Ciencias y Centro de Investigaciónes, Universidad Antonio Nariño, Bogotá; ${ }^{(b)}$ Departamento de Física, Universidad Nacional de Colombia, Bogotá, Colombia; Colombia

$23{ }^{(a)}$ INFN Bologna and Universita' di Bologna, Dipartimento di Fisica; ${ }^{(b)}$ INFN Sezione di Bologna; Italy

24 Physikalisches Institut, Universität Bonn, Bonn; Germany

25 Department of Physics, Boston University, Boston MA; United States of America

26 Department of Physics, Brandeis University, Waltham MA; United States of America

$27{ }^{(a)}$ Transilvania University of Brasov, Brasov; ${ }^{(b)}$ Horia Hulubei National Institute of Physics and Nuclear Engineering, Bucharest; ${ }^{\left({ }^{c}\right)}$ Department of Physics, Alexandru Ioan Cuza University of Iasi, Iasi; ${ }^{(d)}$ National Institute for Research and Development of Isotopic and Molecular Technologies, Physics Department, Cluj-Napoca; ${ }^{(e)}$ University Politehnica Bucharest, Bucharest; ${ }^{(f)}{ }^{\text {West }}$ University in Timisoara, Timisoara; Romania

$28{ }^{(a)}$ Faculty of Mathematics, Physics and Informatics, Comenius University, Bratislava $;^{(b)}$ Department of Subnuclear Physics, Institute of Experimental Physics of the Slovak Academy of Sciences, Kosice; Slovak Republic

29 Physics Department, Brookhaven National Laboratory, Upton NY; United States of America

30 Departamento de Física, Universidad de Buenos Aires, Buenos Aires; Argentina

31 California State University, CA; United States of America

32 Cavendish Laboratory, University of Cambridge, Cambridge; United Kingdom

$33{ }^{(a)}$ Department of Physics, University of Cape Town, Cape Town ${ }^{(b)}$ iThemba Labs, Western Cape; ${ }^{(c)}$ Department of Mechanical Engineering Science, University of Johannesburg,

Johannesburg; ${ }^{(d)}$ University of South Africa, Department of Physics, Pretoria; ${ }^{(e)}$ School of Physics, University of the Witwatersrand, Johannesburg; South Africa

34 Department of Physics, Carleton University, Ottawa ON; Canada

35 (a) Faculté des Sciences Ain Chock, Réseau Universitaire de Physique des Hautes Energies Université Hassan II, Casablanca; ${ }^{(b)}$ Faculté des Sciences, Université Ibn-Tofail, Kénitra; ${ }^{(c)}$ Faculté des Sciences Semlalia, Université Cadi Ayyad, LPHEA-Marrakech; ${ }^{(d)}$ Faculté des Sciences, Université Mohamed Premier and LPTPM, Oujda; ${ }^{(e)}$ Faculté des sciences, Université Mohammed $V$, Rabat; Morocco

36 CERN, Geneva; Switzerland

37 Enrico Fermi Institute, University of Chicago, Chicago IL; United States of America

38 LPC, Université Clermont Auvergne, CNRS/IN2P3, Clermont-Ferrand; France

39 Nevis Laboratory, Columbia University, Irvington NY; United States of America

40 Niels Bohr Institute, University of Copenhagen, Copenhagen; Denmark

$41{ }^{(a)}$ Dipartimento di Fisica, Università della Calabria, Rende $;^{(b)}$ INFN Gruppo Collegato di Cosenza, Laboratori Nazionali di Frascati; Italy

42 Physics Department, Southern Methodist University, Dallas TX; United States of America

43 Physics Department, University of Texas at Dallas, Richardson TX; United States of America

44 National Centre for Scientific Research "Demokritos", Agia Paraskevi; Greece

$45{ }^{(a)}$ Department of Physics, Stockholm University; ${ }^{(b)}$ Oskar Klein Centre, Stockholm; Sweden

46 Deutsches Elektronen-Synchrotron DESY, Hamburg and Zeuthen; Germany

47 Lehrstuhl für Experimentelle Physik IV, Technische Universität Dortmund, Dortmund; Germany

48 Institut für Kern- und Teilchenphysik, Technische Universität Dresden, Dresden; Germany

49 Department of Physics, Duke University, Durham NC; United States of America

50 SUPA - School of Physics and Astronomy, University of Edinburgh, Edinburgh; United Kingdom

51 INFN e Laboratori Nazionali di Frascati, Frascati; Italy

52 Physikalisches Institut, Albert-Ludwigs-Universität Freiburg, Freiburg; Germany

53 II. Physikalisches Institut, Georg-August-Universität Göttingen, Göttingen; Germany 
55 (a) Dipartimento di Fisica, Università di Genova, Genova ${ }^{(b)}$ INFN Sezione di Genova; Italy

56 II. Physikalisches Institut, Justus-Liebig-Universität Giessen, Giessen; Germany

57 SUPA - School of Physics and Astronomy, University of Glasgow, Glasgow; United Kingdom

58 LPSC, Université Grenoble Alpes, CNRS/IN2P3, Grenoble INP, Grenoble; France

59 Laboratory for Particle Physics and Cosmology, Harvard University, Cambridge MA; United States of America

$60{ }^{(a)}$ Department of Modern Physics and State Key Laboratory of Particle Detection and Electronics, University of Science and Technology of China, Hefei $;^{(b)}$ Institute of Frontier and Interdisciplinary Science and Key Laboratory of Particle Physics and Particle Irradiation (MOE), Shandong University, Qingdao; ${ }^{(c)}$ School of Physics and Astronomy, Shanghai Jiao Tong University, KLPPAC-MoE, SKLPPC, Shanghai; ${ }^{(d)}$ Tsung-Dao Lee Institute, Shanghai; China

${ }^{61}{ }^{(a)}$ Kirchhoff-Institut für Physik, Ruprecht-Karls-Universität Heidelberg, Heidelberg; ${ }^{(b)}$ Physikalisches Institut, Ruprecht-Karls-Universität Heidelberg, Heidelberg; Germany

${ }^{62}$ Faculty of Applied Information Science, Hiroshima Institute of Technology, Hiroshima; Japan

${ }^{63}{ }^{(a)}$ Department of Physics, Chinese University of Hong Kong, Shatin, N.T., Hong Kong ${ }^{\left({ }^{(b)}\right.}$ Department of Physics, University of Hong Kong, Hong Kong; ${ }^{(c)}$ Department of Physics and Institute for Advanced Study, Hong Kong University of Science and Technology, Clear Water Bay, Kowloon, Hong Kong; China

${ }^{64}$ Department of Physics, National Tsing Hua University, Hsinchu; Taiwan

${ }^{65}$ IJCLab, Université Paris-Saclay, CNRS/IN2P3, 91405, Orsay; France

66 Department of Physics, Indiana University, Bloomington IN; United States of America

$67{ }^{(a)}$ INFN Gruppo Collegato di Udine, Sezione di Trieste, Udine; ${ }^{(b)}$ ICTP, Trieste; ${ }^{(c)}$ Dipartimento Politecnico di Ingegneria e Architettura, Università di Udine, Udine; Italy

$68{ }^{(a)}$ INFN Sezione di Lecce ${ }^{\left({ }^{(b)}\right.}$ Dipartimento di Matematica e Fisica, Università del Salento, Lecce; Italy

$69{ }^{(a)}$ INFN Sezione di Milano; ${ }^{(b)}$ Dipartimento di Fisica, Università di Milano, Milano; Italy

${ }^{70}{ }^{(a)}$ INFN Sezione di Napoli; ${ }^{(b)}$ Dipartimento di Fisica, Università di Napoli, Napoli; Italy

$71{ }^{(a)}$ INFN Sezione di Pavia; ${ }^{(b)}$ Dipartimento di Fisica, Università di Pavia, Pavia; Italy

$72{ }^{(a)}$ INFN Sezione di Pisa; ${ }^{(b)}$ Dipartimento di Fisica E. Fermi, Università di Pisa, Pisa; Italy

$73{ }^{(a)}$ INFN Sezione di Roma; ${ }^{(b)}$ Dipartimento di Fisica, Sapienza Università di Roma, Roma; Italy

74 (a) INFN Sezione di Roma Tor Vergata; ${ }^{(b)}$ Dipartimento di Fisica, Università di Roma Tor Vergata, Roma; Italy

$75{ }^{(a)}$ INFN Sezione di Roma Tre ${ }^{(b)}$ Dipartimento di Matematica e Fisica, Università Roma Tre, Roma; Italy

$76{ }^{(a)}$ INFN-TIFPA; ${ }^{(b)}$ Università degli Studi di Trento, Trento; Italy

77 Institut für Astro- und Teilchenphysik, Leopold-Franzens-Universität, Innsbruck; Austria

78 University of Iowa, Iowa City IA; United States of America

79 Department of Physics and Astronomy, Iowa State University, Ames IA; United States of America

80 Joint Institute for Nuclear Research, Dubna; Russia

$81{ }^{(a)}$ Departamento de Engenharia Elétrica, Universidade Federal de Juiz de Fora (UFJF), Juiz de Fora ${ }^{(b)}$ Universidade Federal do Rio De Janeiro COPPE/EE/IF, Rio de Janeiro; ${ }^{(c)}$ Universidade Federal de São João del Rei (UFSJ), São João del Rei; ${ }^{(d)}$ Instituto de Física, Universidade de São Paulo, São Paulo; Brazil

82 KEK, High Energy Accelerator Research Organization, Tsukuba; Japan

${ }^{83}$ Graduate School of Science, Kobe University, Kobe; Japan

84 (a) AGH University of Science and Technology, Faculty of Physics and Applied Computer Science, Krakow; ${ }^{(b)}$ Marian Smoluchowski Institute of Physics, Jagiellonian University, Krakow; Poland

85 Institute of Nuclear Physics Polish Academy of Sciences, Krakow; Poland

${ }^{86}$ Faculty of Science, Kyoto University, Kyoto; Japan

87 Kyoto University of Education, Kyoto; Japan

88 Research Center for Advanced Particle Physics and Department of Physics, Kyushu University, 
Fukuoka; Japan

89 Instituto de Física La Plata, Universidad Nacional de La Plata and CONICET, La Plata; Argentina

90 Physics Department, Lancaster University, Lancaster; United Kingdom

91 Oliver Lodge Laboratory, University of Liverpool, Liverpool; United Kingdom

92 Department of Experimental Particle Physics, Jožef Stefan Institute and Department of Physics, University of Ljubljana, Ljubljana; Slovenia

93 School of Physics and Astronomy, Queen Mary University of London, London; United Kingdom

94 Department of Physics, Royal Holloway University of London, Egham; United Kingdom

95 Department of Physics and Astronomy, University College London, London; United Kingdom

96 Louisiana Tech University, Ruston LA; United States of America

97 Fysiska institutionen, Lunds universitet, Lund; Sweden

98 Centre de Calcul de l'Institut National de Physique Nucléaire et de Physique des Particules (IN2P3), Villeurbanne; France

99 Departamento de Física Teorica C-15 and CIAFF, Universidad Autónoma de Madrid, Madrid; Spain

100 Institut für Physik, Universität Mainz, Mainz; Germany

101 School of Physics and Astronomy, University of Manchester, Manchester; United Kingdom

102 CPPM, Aix-Marseille Université, CNRS/IN2P3, Marseille; France

103 Department of Physics, University of Massachusetts, Amherst MA; United States of America

104 Department of Physics, McGill University, Montreal QC; Canada

105 School of Physics, University of Melbourne, Victoria; Australia

106 Department of Physics, University of Michigan, Ann Arbor MI; United States of America

107 Department of Physics and Astronomy, Michigan State University, East Lansing MI; United States of America

108 B.I. Stepanov Institute of Physics, National Academy of Sciences of Belarus, Minsk; Belarus

109 Research Institute for Nuclear Problems of Byelorussian State University, Minsk; Belarus

110 Group of Particle Physics, University of Montreal, Montreal QC; Canada

111 P.N. Lebedev Physical Institute of the Russian Academy of Sciences, Moscow; Russia

112 National Research Nuclear University MEPhI, Moscow; Russia

113 D.V. Skobeltsyn Institute of Nuclear Physics, M.V. Lomonosov Moscow State University, Moscow; Russia

114 Fakultät für Physik, Ludwig-Maximilians-Universität München, München; Germany

115 Max-Planck-Institut für Physik (Werner-Heisenberg-Institut), München; Germany

116 Nagasaki Institute of Applied Science, Nagasaki; Japan

117 Graduate School of Science and Kobayashi-Maskawa Institute, Nagoya University, Nagoya; Japan

118 Department of Physics and Astronomy, University of New Mexico, Albuquerque NM; United States of America

119 Institute for Mathematics, Astrophysics and Particle Physics, Radboud University Nijmegen/Nikhef, Nijmegen; Netherlands

120 Nikhef National Institute for Subatomic Physics and University of Amsterdam, Amsterdam; Netherlands

121 Department of Physics, Northern Illinois University, DeKalb IL; United States of America

$122{ }^{(a)}$ Budker Institute of Nuclear Physics and NSU, SB RAS, Novosibirsk; ${ }^{(b)}$ Novosibirsk State University Novosibirsk; Russia

123 Institute for High Energy Physics of the National Research Centre Kurchatov Institute, Protvino; Russia

124 Institute for Theoretical and Experimental Physics named by A.I. Alikhanov of National Research Centre "Kurchatov Institute", Moscow; Russia

125 Department of Physics, New York University, New York NY; United States of America

126 Ochanomizu University, Otsuka, Bunkyo-ku, Tokyo; Japan

127 Ohio State University, Columbus OH; United States of America

128 Homer L. Dodge Department of Physics and Astronomy, University of Oklahoma, Norman OK; 
United States of America

129 Department of Physics, Oklahoma State University, Stillwater OK; United States of America

130 Palacký University, RCPTM, Joint Laboratory of Optics, Olomouc; Czech Republic

131 Institute for Fundamental Science, University of Oregon, Eugene, OR; United States of America

132 Graduate School of Science, Osaka University, Osaka; Japan

133 Department of Physics, University of Oslo, Oslo; Norway

134 Department of Physics, Oxford University, Oxford; United Kingdom

135 LPNHE, Sorbonne Université, Université de Paris, CNRS/IN2P3, Paris; France

136 Department of Physics, University of Pennsylvania, Philadelphia PA; United States of America

137 Konstantinov Nuclear Physics Institute of National Research Centre "Kurchatov Institute", PNPI, St. Petersburg; Russia

138 Department of Physics and Astronomy, University of Pittsburgh, Pittsburgh PA; United States of America

$139{ }^{(a)}$ Laboratório de Instrumentação e Física Experimental de Partículas - LIP, Lisboa; ${ }^{(b)}$ Departamento de Física, Faculdade de Ciências, Universidade de Lisboa,

Lisboa; ${ }^{(c)}$ Departamento de Física, Universidade de Coimbra, Coimbra; ${ }^{(d)}$ Centro de Física Nuclear da Universidade de Lisboa, Lisboa; ${ }^{(e)}$ Departamento de Física, Universidade do Minho,

Braga: ${ }^{(f)}$ Departamento de Física Teórica y del Cosmos, Universidad de Granada, Granada (Spain); ${ }^{(g)}$ Dep Física and CEFITEC of Faculdade de Ciências e Tecnologia, Universidade Nova de Lisboa, Caparica; ${ }^{(h)}$ Instituto Superior Técnico, Universidade de Lisboa, Lisboa; Portugal

140 Institute of Physics of the Czech Academy of Sciences, Prague; Czech Republic

141 Czech Technical University in Prague, Prague; Czech Republic

142 Charles University, Faculty of Mathematics and Physics, Prague; Czech Republic

143 Particle Physics Department, Rutherford Appleton Laboratory, Didcot; United Kingdom

144 IRFU, CEA, Université Paris-Saclay, Gif-sur-Yvette; France

145 Santa Cruz Institute for Particle Physics, University of California Santa Cruz, Santa Cruz CA; United States of America

$146{ }^{(a)}$ Departamento de Física, Pontificia Universidad Católica de Chile, Santiago; ${ }^{(b)}$ Universidad Andres Bello, Department of Physics, Santiago; ${ }^{(c)}$ Instituto de Alta Investigación, Universidad de Tarapacá; ${ }^{(d)}$ Departamento de Física, Universidad Técnica Federico Santa María, Valparaíso; Chile Department of Physics, University of Washington, Seattle WA; United States of America

148 Department of Physics and Astronomy, University of Sheffield, Sheffield; United Kingdom

149 Department of Physics, Shinshu University, Nagano; Japan

150 Department Physik, Universität Siegen, Siegen; Germany

151 Department of Physics, Simon Fraser University, Burnaby BC; Canada

152 SLAC National Accelerator Laboratory, Stanford CA; United States of America

153 Physics Department, Royal Institute of Technology, Stockholm; Sweden

154 Departments of Physics and Astronomy, Stony Brook University, Stony Brook NY; United States of America

155 Department of Physics and Astronomy, University of Sussex, Brighton; United Kingdom

156 School of Physics, University of Sydney, Sydney; Australia

157 Institute of Physics, Academia Sinica, Taipei; Taiwan

$158{ }^{(a)}$ E. Andronikashvili Institute of Physics, Iv. Javakhishvili Tbilisi State University, Tbilisi $;^{(b)}$ High Energy Physics Institute, Tbilisi State University, Tbilisi; Georgia

159 Department of Physics, Technion, Israel Institute of Technology, Haifa; Israel

160 Raymond and Beverly Sackler School of Physics and Astronomy, Tel Aviv University, Tel Aviv; Israel

161 Department of Physics, Aristotle University of Thessaloniki, Thessaloniki; Greece

162 International Center for Elementary Particle Physics and Department of Physics, University of Tokyo, Tokyo; Japan

163 Graduate School of Science and Technology, Tokyo Metropolitan University, Tokyo; Japan

164 Department of Physics, Tokyo Institute of Technology, Tokyo; Japan 
State University, Tomsk; Russia

166 Department of Physics, University of Toronto, Toronto ON; Canada

$67{ }^{(a)}$ TRIUMF, Vancouver BC; ${ }^{(b)}$ Department of Physics and Astronomy, York University, Toronto ON; Canada

168 Division of Physics and Tomonaga Center for the History of the Universe, Faculty of Pure and Applied Sciences, University of Tsukuba, Tsukuba; Japan

169 Department of Physics and Astronomy, Tufts University, Medford MA; United States of America

170 Department of Physics and Astronomy, University of California Irvine, Irvine CA; United States of America

171 Department of Physics and Astronomy, University of Uppsala, Uppsala; Sweden

172 Department of Physics, University of Illinois, Urbana IL; United States of America

173 Instituto de Física Corpuscular (IFIC), Centro Mixto Universidad de Valencia - CSIC, Valencia; Spain

174 Department of Physics, University of British Columbia, Vancouver BC; Canada

175 Department of Physics and Astronomy, University of Victoria, Victoria BC; Canada

176 Fakultät für Physik und Astronomie, Julius-Maximilians-Universität Würzburg, Würzburg; Germany

177 Department of Physics, University of Warwick, Coventry; United Kingdom

178 Waseda University, Tokyo; Japan

179 Department of Particle Physics, Weizmann Institute of Science, Rehovot; Israel

180 Department of Physics, University of Wisconsin, Madison WI; United States of America

181 Fakultät für Mathematik und Naturwissenschaften, Fachgruppe Physik, Bergische Universität Wuppertal, Wuppertal; Germany

182 Department of Physics, Yale University, New Haven CT; United States of America

a Also at Borough of Manhattan Community College, City University of New York, New York NY; United States of America

b Also at Centro Studi e Ricerche Enrico Fermi; Italy

c Also at CERN, Geneva; Switzerland

d Also at CPPM, Aix-Marseille Université, CNRS/IN2P3, Marseille; France

e Also at Département de Physique Nucléaire et Corpusculaire, Université de Genève, Genève; Switzerland

f Also at Departament de Fisica de la Universitat Autonoma de Barcelona, Barcelona; Spain

$g$ Also at Department of Applied Physics and Astronomy, University of Sharjah, Sharjah; United Arab Emirates

h Also at Department of Financial and Management Engineering, University of the Aegean, Chios; Greece

i Also at Department of Physics and Astronomy, Michigan State University, East Lansing MI; United States of America

j Also at Department of Physics and Astronomy, University of Louisville, Louisville, KY; United States of America

* Also at Department of Physics, Ben Gurion University of the Negev, Beer Sheva; Israel

${ }^{l}$ Also at Department of Physics, California State University, East Bay; United States of America

$m$ Also at Department of Physics, California State University, Fresno; United States of America

$n$ Also at Department of Physics, California State University, Sacramento; United States of America

o Also at Department of Physics, King's College London, London; United Kingdom

${ }^{p}$ Also at Department of Physics, St. Petersburg State Polytechnical University, St. Petersburg; Russia

q Also at Department of Physics, University of Adelaide, Adelaide; Australia

$r$ Also at Department of Physics, University of Fribourg, Fribourg; Switzerland

s Also at Dipartimento di Matematica, Informatica e Fisica, Università di Udine, Udine; Italy

${ }^{t}$ Also at Faculty of Physics, M.V. Lomonosov Moscow State University, Moscow; Russia 
u Also at Giresun University, Faculty of Engineering, Giresun; Turkey

$v$ Also at Graduate School of Science, Osaka University, Osaka; Japan

w Also at Hellenic Open University, Patras; Greece

${ }^{x}$ Also at IJCLab, Université Paris-Saclay, CNRS/IN2P3, 91405, Orsay; France

y Also at Institucio Catalana de Recerca $i$ Estudis Avancats, ICREA, Barcelona; Spain

$z$ Also at Institut für Experimentalphysik, Universität Hamburg, Hamburg; Germany

aa Also at Institute for Mathematics, Astrophysics and Particle Physics, Radboud University Nijmegen/Nikhef, Nijmegen; Netherlands

ab Also at Institute for Nuclear Research and Nuclear Energy (INRNE) of the Bulgarian Academy of Sciences, Sofia; Bulgaria

ac Also at Institute for Particle and Nuclear Physics, Wigner Research Centre for Physics, Budapest; Hungary

ad Also at Institute of Particle Physics (IPP), Vancouver; Canada

ae Also at Institute of Physics, Azerbaijan Academy of Sciences, Baku; Azerbaijan

af Also at Instituto de Fisica Teorica, IFT-UAM/CSIC, Madrid; Spain

ag Also at Joint Institute for Nuclear Research, Dubna; Russia

ah Also at Louisiana Tech University, Ruston LA; United States of America

ai Also at Moscow Institute of Physics and Technology State University, Dolgoprudny; Russia

aj Also at National Research Nuclear University MEPhI, Moscow; Russia

ak Also at Physics Department, An-Najah National University, Nablus; Palestine

al Also at Physikalisches Institut, Albert-Ludwigs-Universität Freiburg, Freiburg; Germany

am Also at The City College of New York, New York NY; United States of America

an Also at TRIUMF, Vancouver BC; Canada

ao Also at Universita di Napoli Parthenope, Napoli; Italy * Deceased 